University of South Carolina

Scholar Commons

Theses and Dissertations

$1-1-2013$

\title{
Three-Dimensional Evolution of Mechanical Percolation in Nanocomposites with Random Microstructures
}

Bethany Suzanne Fralick

University of South Carolina

Follow this and additional works at: https://scholarcommons.sc.edu/etd

Part of the Mechanical Engineering Commons

\section{Recommended Citation}

Fralick, B. S.(2013). Three-Dimensional Evolution of Mechanical Percolation in Nanocomposites with Random Microstructures. (Doctoral dissertation). Retrieved from https://scholarcommons.sc.edu/etd/ 2230

This Open Access Dissertation is brought to you by Scholar Commons. It has been accepted for inclusion in Theses and Dissertations by an authorized administrator of Scholar Commons. For more information, please contact digres@mailbox.sc.edu. 
Three-Dimensional EVOLUtion of MECHANiCAL PERCOLATION IN NANOCOMPOSITES WITH RANDOM MICROSTRUCTURES

by

Bethany Suzanne Fralick

Bachelor of Science

Miami University 2007

Master of Science

University of South Carolina 2009

Submitted in Partial Fulfillment of the Requirements

for the Degree of Doctor of Philosphy in

Mechanical Engineering

College of Engineering and Computing

University of South Carolina

2013

Accepted by:

Sarah Baxter, Major Professor

Jed Lyons, Committee Member

Xiaodong Li, Committee Member

Edward Gatzke, Committee Member

Lacy Ford, Vice Provost and Dean of Graduate Studies 
(C) Copyright by Bethany Suzanne Fralick, 2013

All Rights Reserved. 


\section{DEDICATION}

I dedicate this work to my family.

My father, who realized my talent.

My mother, who reminded me of patience.

My sister, who laughed alongside me.

My brother, who provided stress relief.

My best friend, who gave me courage.

XO 


\section{ACKNOWLEDGMENTS}

Thank you to my family and friends for not only their professional support, but also their social support during my doctoral education. I would like to thank my advisor, Dr. Sarah Baxter, for her guidance throughout this mentorship. Thank you to my committee for their extra, valuable time and effort during the recent years. I would like to also thank the mechanical engineering department and the wonderful people that aid in its success. I would like to acknowledge the National Science Foundation; this work has been supported by CMMI \#1030162. I would like to thank NASAGlenn and Dr. Steve Arnold for the GMC-MAC software. 


\begin{abstract}
One mechanism that is expected to play a large role in the enhanced, and sometimes novel, mechanical properties of nanocomposites is the probabilistic formation of percolated or connected microstructures. The majority of the models used to describe mechanical percolation have the functional form of a power law and depend on prior knowledge of a percolation threshold or critical volume fraction. While these models have been fairly accurate predictors of electrical conductivity in composites, they do not take any microstructural mechanisms, other than connectivity, into consideration. Classic mean-field micromechanics models, however, do not capture the variability in effective properties due to a random microstructure. In this work, aspects of both modeling approaches, i.e. probabilistic events and micromechanics, are adopted. A computational unit cell model is used to calculate the effective composite properties of random microstructures based on principles of micromechanics. The influence of the spatial randomness is incorporated using Monte Carlo techniques to simulate microstructural realizations. In this way, the modeling paradigm is reversed. Instead of using a percolation threshold to predict mechanical properties, mechanical properties are used to demonstrate the location of apparent percolation thresholds. By observing the distributions and variations of the predicted effective properties, the evolution of microstructural events can be tracked.

Microstructures were simulated for a model material system consisting of metallic particles in a polymer matrix. Effects of a matrix-particle interface, interfacial thickness and interfacial stiffness, were also considered. The influence of particle aspect ratio on the apparent percolation threshold was also explored.
\end{abstract}




\section{Contents}

Dedichtion ............................... ii

ACKNOWLEDGMENTS ..................... . . . iv

AbStract . . . . . . . . . . . . . . . . . . . . . . . . . . . $\mathrm{v}$

List of FigurES . . . . . . . . . . . . . . . . . . . . . . . viii

Chapter 1 Introduction . . . . . . . . . . . . . . . . . . . . . 1

Chapter 2 Background and Review of the Literature . . . . . . 5

Chapter 3 Computational Modeling . . . . . . . . . . . . . 10

3.1 Micromechanics . . . . . . . . . . . . . . . . . . 10

3.2 Percolation Models . . . . . . . . . . . . . . . . 15

3.3 Monte Carlo Simulations . . . . . . . . . . . . . . . . . 16

Chapter 4 Mechanical Percolation in Two-Phase Composite;

Cubic Particles . . . . . . . . . . . . . . . . . . . 18

Chapter 5 Mechanical Percolation in Three-Phase Composite; Cubic Particles . . . . . . . . . . . . . . . . . . 25

Chapter 6 Effect of Interfacial Stiffness and Thickness . . . . 30

6.1 Stiffness Variations . . . . . . . . . . . . . . . . . . . 31

6.2 Thickness Variations . . . . . . . . . . . . . . . . 35

6.3 Functionally Graded Interface . . . . . . . . . . . . . . . . . . 39 
6.4 Summary and Conclusions . . . . . . . . . . . . . . . . . 40

Chapter 7 Two- and Three-Phase Continuum Model $\ldots \ldots \ldots$

7.1 Continuum versus Lattice . . . . . . . . . . . . . 46

Chapter 8 High Aspect Ratio Particles . . . . . . . . . . . . . 49

8.1 Two-Phase Composite with Varying Aspect Ratios . . . . . . . . . . 49

8.2 Three-Phase Composite, Influence of Aspect Ratio . . . . . . . . . 50

Chapter 9 Summary and Discussion $\ldots \ldots \ldots \ldots \ldots$

Chapter 10 Future Work . . . . . . . . . . . . . . . . . 62

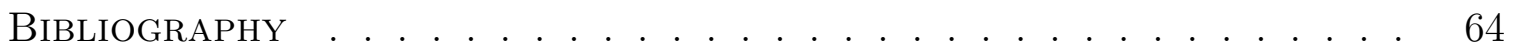

Appendix A Matlab Simulation Analysis Code . . . . . . . . 69

Appendix B Matlab RUC Generation Code. . . . . . . . . . 79

Appendix C Example GMC Input Code . . . . . . . . . . . 83 


\section{List OF Figures}

Figure 2.1 Distinctive shape of ideal percolation curve; log of effective properties, $M^{*}$, versus volume fraction. Sharp increase in properties at the critical volume fraction $\phi_{c} \ldots \ldots . \ldots 6$

Figure 3.1 GMC's Repeating Unit Cell with white matrix subcells and black particle subcells. . . . . . . . . . . . . . . . . . 12

Figure 4.1 Effective elastic axial stiffnesses for the simulated two-phase composite; normalized minimum, average, and maximum values. Simulations based on random microstructure are compared to the Mori-Tanaka micromechanics model. . . . . . . . . . . . . 19

Figure 4.2 Distributions of axial moduli in the two-phase composite at various volume fractions: a) 0.60 b) 0.65 c) 0.70 d) 0.75 e) 0.80

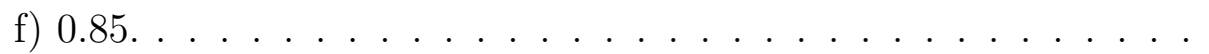

Figure 4.3 Comparison of simulated effective elastic axial stiffness for twophase composite; normalized minimum, average, and maximum values, to classic percolation model based on eqn.(2.3), with $\phi_{c}=0.60, a=0.65$, and $f=3.75$; and series parallel model based on eqn. $(2.3,2.4)$, with $b=6.0 \ldots \ldots 23$

Figure 4.4 Percent of simulated microstructures in the two-phase composite that have continuous stacks. . . . . . . . . . . . .

Figure 5.1 Simulated effective elastic stiffness for three-phase composite; normalized minimum, average, and maximum values. . . . . . . 
Figure 5.2 Matrix and interface volume fraction versus particle volume fraction; simulation values and ideal predictions. . . . . . . . . 27

Figure 5.3 Distributions of shear moduli in the three-phase composite. In comparison, there is no variation in the shear modulus in the two-phase composite. . . . . . . . . . . . . . . 28

Figure 6.1 Simulated effective elastic axial stiffness (normalized minimum, average, and maximum values) for top: [Compliant, Thin]; middle: [Compliant, Original Thickness]; and bottom: [Compliant, Thick] . .

Figure 6.2 Simulated effective elastic axial stiffness (normalized minimum, average, and maximum values) for top: [Original Stiffness, Thin]; middle: [Original Stiffness, Original Thickness]; and bottom: [Original Stiffness, Thick $] \ldots \ldots \ldots \ldots \ldots$

Figure 6.3 Simulated effective elastic axial stiffness (normalized minimum, average, and maximum values) for top: [Stiff, Thin]; middle: [Stiff, Original Thickness]; and bottom: [Stiff, Thick] . . . . . . . . 34

Figure 6.4 Simulated effective elastic axial stiffness (normalized minimum, average, and maximum values) for top: [Thin, Compliant]; middle: [Thin, Original Stiffness]; and bottom: [Thin, Stiff] . . . . . . . . 36

Figure 6.5 Simulated effective elastic axial stiffness (normalized minimum, average, and maximum values) for top: [Original Thickness, Compliant]; middle: [Original Thickness, Original Stiffness]; and bottom: [Original Thickness, Stiff] . . . . . . . . . . . . . . . 37

Figure 6.6 Simulated effective elastic axial stiffness (normalized minimum, average, and maximum values) for top: [Thick, Compliant]; middle: [Thick, Original Stiffness]; and bottom: [Thick, Stiff] . . . . . . . . 38

Figure 6.7 Simulated effective elastic axial stiffness (normalized minimum, average, and maximum) for five-phase composite with a gradient interface. 39 
Figure 6.8 Simulated effective elastic axial stiffness for five-phase composite with a gradient interface; three-phase composite with a thick, original stiffness interface; and three-phase compsite with a thin, stiff interface. . . . . . . . . . . . . . . . . 40

Figure 6.9 Simulated average effective elastic axial stiffnesses for three-phase composite in top: compliant group; middle: original stiffness group; and top: stiff group. . . . . . . . . . . . . . . . . 41

Figure 6.10 Simulated average effective elastic axial stiffnesses for three-phase composite in top: thin variation; middle: original thickness variation; and top: thick variation. . . . . . . . . . . . . . . . 43

Figure 7.1 Examples of possible overlap scenarios within the RUC . . . . . . 46

Figure 7.2 Two-phase $2 \times 2 \times 2$ particle composite: Continuum and Lattice model comparison. . . . . . . . . . . . . . . . . . . 47

Figure 7.3 Three-phase $2 \times 2 \times 2$ particle composite: Continuum and Lattice model comparison. . . . . . . . . . . . . . . . . . . . . . 48

Figure 8.1 Simulated effective elastic axial stiffness for two-phase composite with 6:1 aspect ratio particles, longitudinal and transverse overlap. . .

Figure 8.2 Simulated effective elastic axial stiffness for two-phase composite with 8:1 aspect ratio particles, longitudinal and transverse overlap. . . 51

Figure 8.3 Simulated effective elastic axial stiffness for two-phase composite with 12:1 aspect ratio particles, longitudinal and transverse overlap. $\quad . \quad 52$

Figure 8.4 Probability of percolated microstructures for two-phase composite with particles of various aspect ratio. . . . . . . . . . . . 52

Figure 8.5 Simulated effective elastic axial stiffness for two-phase composite containing particles with top: 5:1 aspect ratio; middle: 7:1 aspect ratio; and bottom: 10:1 aspect ratio. . . . . . . . . . . . . 
Figure 8.6 Simulated effective elastic axial stiffness for three-phase composite with 3:1 aspect ratio particles, longitudinal and transverse overlap. . .

Figure 8.7 Simulated effective elastic axial stiffness for three-phase composite with 5:1 aspect ratio particles, longitudinal and transverse overlap. . .

Figure 8.8 Simulated effective elastic axial stiffness averages for three-phase composite with 5:1 aspect ratio particles, longitudinal and transverse overlap. . . . . . . . . . . . . . . . . . . . .

Figure 8.9 Simulated effective elastic axial stiffness for three-phase composite with 8:1 aspect ratio particles, longitudinal and transverse overlap. . . 56

Figure 8.10 Simulated effective elastic axial stiffness averages for three-phase composite with $3: 1,5: 1$, and 8:1 aspect ratio particles, longitudinal and transverse overlap. . . . . . . . . . . . . . . 56 


\section{Chapter 1}

\section{INTRODUCTION}

One mechanism that is expected to play a large role in the enhanced mechanical properties of nanocomposites is the formation of percolated microstructures. These connected structures form as a result of randomness in the spatial arrangement of the included composite phase and are defined in terms of the probability that they will form at a given volume fraction. The majority of the models used to describe percolation have the functional form of a power law and depend on prior knowledge of the volume fraction where the connected structure is most likely to form; this is referred to as a percolation threshold or critical volume fraction. These models have been fairly accurate predictors of electrical conductivity in composites. Because effects which resemble the trends for percolation of electrical conductivity have also been observed for mechanical properties and are commonly referred to as 'mechanical

percolation', the same power-law models have been used in modeling. Power-law models, however, do not take any microstructural mechanisms other than connectivity into consideration.

In theory, composite electrical conductivity, with respect to volume fraction, is relatively binary; below a threshold volume fraction the composite is not conductive, or has some base-line conductivity, and at some point, at or above the threshold, its conductivity is greatly enhanced. Mechanical percolation, however, necessarily occurs in tandem with other microstructural mechanisms and is perhaps better described as a continuous process. This is the challenge in modeling the effects of mechanical percolation. Certainly a connected microstructure will enhance mechanical properties, 
and it would be expected that the difference between a connected matrix phase and a connected particle phase would be dramatic. But there are additional mechanisms, beyond connectivity, at play. In contrast to conductivity, composite mechanical response is dominated by the volume fraction of the included phase so that, even prior to the formation of a connected phase, composite properties are affected by the amount of filler. In addition, if a less stiff matrix is confined between stiffer, closely packed, although not connected, regions of an included phase, the ability of the matrix to deform may be reduced, making it effectively stiffer. Thus, while percolation thresholds are still critical to characterizing these materials, the mechanical response may depend on a combination of mechanisms.

In the case of polymer nanocomposites, interest in mechanical percolation has perhaps become greater due to the anticipated significant effect of a matrix-filler interface region $[1,2]$. This interface region is due to a perturbation of the matrix material resulting from the presence of the particles; e.g. the result of bonding between the phases, confinement of the matrix between stiffer regions, or interference in the mobility of the long flexible chains of the polymer, which result in a localized increase in matrix stiffness. This local effect is present in all composite materials, but because of the high surface area to volume ratio for nanoscale inclusions, the interface region in nanocomposites can constitute a significant third phase of the composite. As an example, an interface with a thickness of 15 nanometers surrounding a 100 micrometer diameter particle will have little influence on effective composite properties; this same thickness surrounding a 30 nanometer diameter particle may represent a volume fraction equal to or greater than that of the particles.

With respect to mechanical percolation, the presence of an interfacial region suggests that two levels of percolation may develop. For the first level the interface region may assist in the formation a connected structure (particles and interface) prior to, i.e. at a much lower volume fraction, than the second level, where the par- 
ticles alone percolate. In [3], investigators included interface regions in an extension of the excluded volume model, [4]. Tighter and lower bounds were developed for the critical volume fraction of random, high aspect ratio nano-fiber composites. These bounds captured the effect of pseudo-percolation, defined as where the interface and particles combine to form a connected microstructural phase. The narrower bounds corresponded well to experimental observation.

In the second range, at high volume fractions, regions with matrix properties are completely replaced by regions with interface properties. Within what is now a two-phase composite, interface and particles, more classic percolation is expect to occur.

In this work, rather than attempting to estimate critical volume fractions a priori, effective elastic composite properties were used to track the effects of the evolution of a mechanically percolating microstructure. This was done through simulations of the effective properties for multiple realizations of a random microstructure. Simulations were performed for both a macro-scale and nano-scale discontinuously reinforced composite. The two are distinguished by the inclusion of an appropriately scaled interface region in the nanocomposite model. Representative volume elements (RVEs), describing random arrangements of geometrically isotropic particles (the included phase is modeled as cubes) in a more compliant matrix were simulated over a range of volume fractions. These RVEs were used as the repeating unit cell (RUC) in the periodic unit cell micromechanics model, known as the Generalized Method of Cells (GMC). GMC was then used to predict the effective elastic composite properties for each microstructural realization. Minimum, average, and maximum values of the distribution of effective properties, for each volume fraction, are used to characterize trends.

In Chapter 2, a review of literature in pertinent research is presented. In Chapter 3 , the computational models used in this work are described. This includes a 
brief description of GMC, with the underlying assumptions required in using it to model random microstructures, and its use in combination with Monte Carlo Methods. Chapter 4 presents data and analysis from simulations of a two-phase composite; particles and matrix. These model results are based on the same framework as the lattice-grid models used in classic percolation theory. These results are compared to a mean field micromechanics model in order to illustrate the differences between this approach and standard predictions of mechanical response. Chapter 5 presents results and analysis for a three-phase material, representing a nanocomposite; particles, interface, and matrix, again in a lattice-grid framework. In Chapter 6, results and analysis regarding the effects of the composite interface elastic stiffness and thickness are presented. Chapter 7 discusses the extension of the method from a lattice grid model to a more continuum model of percolation, where particles can overlap what were originally lattice sites. Chapter 8 offers analysis and results of higher aspect ratio particles in a two- and three-phase composite, in the continuum framework. Chapter 9 draws some conclusions and discusses the significance of this work andChapter 10 suggests future work. 


\section{CHAPTER 2}

\section{BACKGROUND AND REVIEW OF THE Literature}

Classic percolation is framed on a discrete lattice grid; sites on the lattice can be occupied or unoccupied. The probability of a site being filled is $p$; the probability of it being unfilled is $(1-p)$. The problem asks, for each number of occupied sites, what is the probability that a random filling of the lattice results in a connected series of grid points, i.e. a percolated network. The dual problem frames connectivity with respect to bonds, or connections between sites, on a grid. A critical threshold, or fraction, of occupied sites/bonds can be identified below which the system will seldom percolate, but above which it almost always does. Site/bond percolation is referred to as discrete percolation, as only site locations can be occupied. In physics and engineering, percolation theory has been extensively applied to modeling electrical conductivity in composites. In many cases these applications are more accurately described by continuum percolation, where off-lattice sites can be occupied. For composites, the percolation threshold is characterized by the volume fraction of an included phase.

For electrical conductivity, the percolation concept is intuitive. As an increasing volume of an electrically conductive filler is dispersed in an insulating matrix, the composite material will, at a critical volume fraction, $\phi_{c}$, exhibit a sharp increase in conductivity. This critical value corresponds to the point where the probability is high that the included particles transform from well dispersed filler to spanning clusters that form a connected electrical pathway. The conductivity, as the volume 


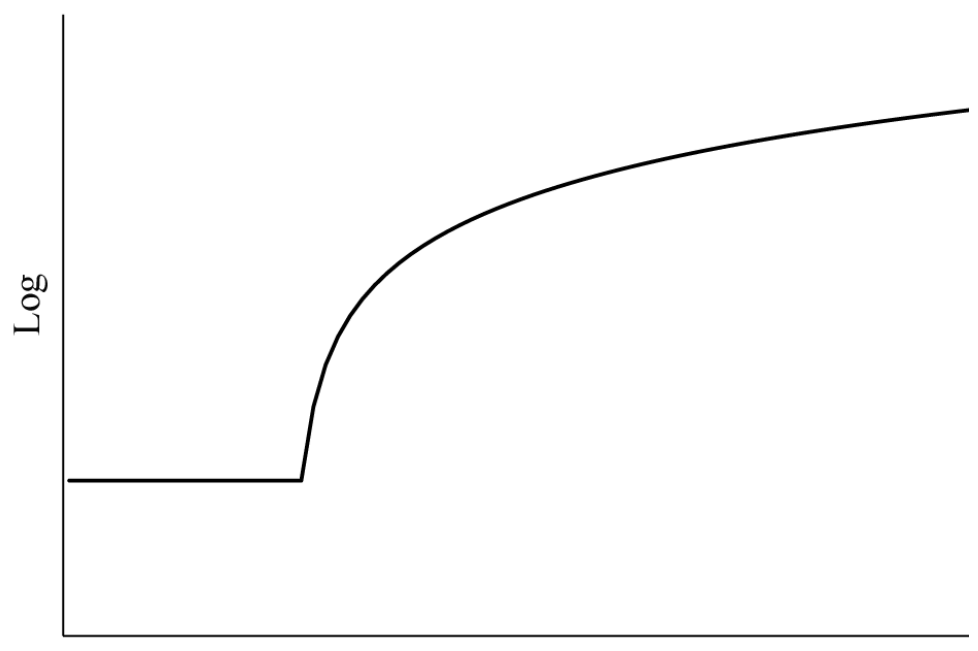

Volume Fraction

Figure 2.1: Distinctive shape of ideal percolation curve; log of effective properties, $M^{*}$, versus volume fraction. Sharp increase in properties at the critical volume fraction $\phi_{c}$.

fraction reaches and exceeds this value, is often modeled by a power-law,

$$
\sigma=\sigma_{0}\left(\phi-\phi_{c}\right)^{b},
$$

where $\sigma$ is the conductivity of the composite, $\phi$ and $\phi_{c}$ are volume fraction and critical volume fraction respectively, $\sigma_{0}$ is the conductivity of the filler and $b$ is the critical exponent $[5,6]$.

The majority of the research into connected or percolated microstructures has focused on establishing this critical volume fraction, or percolation threshold. Thresholds have been experimentally determined through observations of the location of a jump in the composite conductivity. This effect is characterized on semi-log plots of conductivity versus volume fraction by the distinct shape of a power-law curve, see fig.2.1.

The mathematics of percolation has also included simulations of two- and threedimensional random arrangements of discrete particle shapes coupled with computational evaluations of connectivity, [7]-[11], as well as studies into the statistical and 
mathematical basis [12]-[15] of both discrete and continuum percolation models. The critical exponents are generally universal constants, as they are independent of the lattice structure and microscopic details. They vary only for the dimensionality of the system. This universality appears to hold for both discrete and continuum percolation, although the scaling factors (e.g., $\sigma_{0}$ ) do depend on the system and are not universal, $[16,17]$. When effective elastic properties are considered, the critical exponent appears to no longer be universal. This is likely due to the higher tensorial order of the elastic problem. The elastic stiffness tensor is a $4^{\text {th }}$ order tensor, the electrical conductivity tensor is a $2^{\text {nd }}$ order tensor, [15].

The percolation threshold, $\phi_{c}$, can take on any value between zero and one for systems with dimension $d \geq 2$. It is a non-universal value that depends on dimension $d$, lattice structure and other microscopic details such as interactions [15]. Exact values can be determined for only a few two-dimensional lattices. In general, estimates are derived through numerical approximation and simulation. Continuum models can include overlap and penetration effects which introduce additional variables, [15].

In the early 80 's Balberg et al. $[7,12,13]$ extended the study of percolation thresholds from point-based lattice models to anisotropically shaped objects, e.g., rods. They simulated percolation of high aspect ratio 'sticks' considering a distribution of lengths and various angle orientations, in two and three dimensions, therefore making the estimates more relevant to the mechanics of composite materials. In particular, they extended the work of Scher and Zallen [14] on the fractional area and volume of the included phase associated with the onset of percolation, and derived expressions for average total excluded area and total excluded volume, resulting in bounds on critical volume fractions.

Similar sharp increases in mechanical properties with volume fraction have also been experimentally observed and linked to the formation of connected microstructures. In particular, mechanical percolation has been notable in composites contain- 
ing high aspect ratio fillers, as well as frequently observed in nanocomposites, see for example [18]-[21].

Estimates of $\phi_{c}$ used in modeling mechanical percolation have, in general, derived from the same theory and simulations developed for conductivity models. Often electrical and mechanical properties are studied in the same material, e.g., [22, 23], where measurements of conductivity are used to establish threshold values, which are then used in models that predict mechanical properties. These micromechanical models are often based on a scaled version of the power law model, with the elastic modulus replacing the conductivity term, eqn.2.2

$$
\sigma(p) \sim\left(p-p_{c}\right)^{t}
$$

where $t$ is the critical exponent of conductivity. Studies have estimated values of $t$ ranging from 1.1 to 1.38 [24]-[26]. Watson and Leath [24] used table-top experiments while Straley [25] and Yuge and Onizuka [26] reported on square and simple cubic lattice by means of an over-relaxation procedure [27].

One version of a classic percolation model for elastic moduli, which models the effective composite modulus, $E^{*}$, over the full range of volume fractions, is

$$
E^{*}(v)= \begin{cases}E_{m}\left(\phi_{c}-v\right)^{-a} & \text { if } v \leq \phi_{c} \\ E_{p}\left(v-\phi_{c}\right)^{f} & \text { if } \quad \phi_{c}<v,\end{cases}
$$

where $E_{m}, E_{p}$ are the moduli of the matrix and particle phases and $v$ is the volume fraction of the filler. Suggested magnitudes for the exponents vary in the literature. A model that captures the continuum, non-decreasing stiffness in the initial, low volume fraction regime requires that the first formula have a negative exponent $a$, as shown in eq. (2.3). This differs from the form suggested in the literature.

Other models use the power law form to estimate an intermediate parameter which can then be embedded in models of effective properties. For example, the 
phenomenological series/parallel model, $[18,19]$ for composite elastic modulus, $E^{*}$, valid for $v \geq \phi_{c}$, is given by

$$
E^{*}(v)=\frac{(1-2 \phi+\phi v) E_{p} E_{m}+(1-v) \phi E_{m}^{2}}{(1-v) E_{p}+(v-\phi) E_{m}},
$$

where $\phi$ approximates the volume fraction of the filler actually responsible for the rigidity of the composite as

$$
\phi=v\left[\frac{\left.v-\phi_{c}\right)}{1-\phi_{c}}\right]^{b}, \text { for } v>\phi_{c}
$$

and $b$ is again a percolation exponent.

Possibly the dominant model in the literature is the General Effective Media (GEM) Model developed by McLachlan [28, 29]. This model was originally developed for electrical composites and represents an interpolation between the Bruggerman symmetric model based on electric dipoles and classic percolation. GEM is defined by

$$
(1-f) \frac{\sigma_{m}^{1 / t}-\sigma_{c}^{1 / t}}{\sigma_{m}^{1 / t}+A \sigma_{c}^{1 / t}}+f \frac{\sigma_{p}^{1 / t}-\sigma_{c}^{1 / t}}{\sigma_{p}^{1 / t}+A \sigma_{c}^{1 / t}}=0,
$$

where $f$ is the volume fraction; $\sigma_{m}, \sigma_{p}$, and $\sigma_{c}$ are the conductivities of the matrix, particle, and composite, respectively; and A is a constant,

$$
A=\frac{1-\phi_{c}}{\phi_{c}} .
$$

What all of these models have in common is the requirement that the percolation threshold be known before properties can be estimated. Since a connected microstructure is the dominant mechanism for electrical conductivity, percolation thresholds estimated on these grounds are appropriate. For mechanical percolation, the theoretical bounds do not correspond well to observed experimental results and so often the models are used empirically, fitting the curve to the data. 


\section{Chapter 3}

\section{Computational Modeling}

The computational model in this work is based on two numerical techniques. First, the effective elastic properties of a composite were calculated using the computational micromechanics model known as the Generalized Method of Cells. The geometry of this micromechanics models can be compared to mathematical percolation frameworks. Second, the range of properties associated with a spatially random microstructure were characterized by the results of Monte Carlo simulations of microstructures.

\subsection{MiCROMECHANICS}

To predict the effective elastic properties of the composite, the three-dimensional micromechanics model, known as the Generalized Method of Cells (GMC) [30], was used. GMC is a periodic unit cell model that uses a rectangular repeating unit cell (RUC), composed of multiple subcells, as the representative volume element (RVE) for a periodic microstructure, see fig.3.1. The arbitrary number of subcells, each assigned properties of one of the composite phases, provides a significant amount of microstructural detail. The homogenization process in GMC connects the material microstructure, through periodic boundary conditions, to an equivalent homogeneous material with a set of continuum level equations; resulting in a prediction of the effective properties. Specific boundary conditions enforce continuity of displacements and tractions across subcell boundaries and between RUCs. As these conditions are satisfied in an average sense, integrating over the boundaries, the effect of the 
rectangular geometry, i.e. sharp corners, is minimal in the prediction of effective properties.

GMC's predictions of the effective elastic properties compare well to the classic Mori-Tanaka model (MT), [31, 32] for two-phase composites [33], in the low volume fraction range where MT is assumed most valid. GMC's advantage, in particular for modeling an interface region, is that it includes a description of the relative spatial position of the included phase, i.e., an interface region surrounding a particle. These effects result in more pronounced differences between the two models at higher volume fractions. A comparison of differences between GMC and MT resulting from this more detailed spatial description, in a deterministic setting, is presented in [34]. A more efficient implementation of GMC, [35], has significantly reduced computation time for complex microstructures. GMC has also been used effectively to characterize random microstructures using a moving window analysis, e.g., [36]-[39].

The GMC model begins with a repeating unit cell. The unit cell is divided into $\left(N_{\alpha} \times N_{\beta} \times N_{\gamma}\right)$ rectangular subcells, where the number of subcells is determined by the detail needed to describe the microstructure. Each subcell is homogeneous and contains one of the constituent components of the heterogeneous material. A subcell is denoted by the ordered triple $(\alpha, \beta, \gamma)$ that identifies its $x_{1}$ position, from bottom to top, its $x_{2}$ position, left to right, and its $x_{3}$ position, as shown in fig. 3.1. Each subcell volume is given by $d_{\alpha} \times h_{\beta} \times l_{\gamma}$.

GMC establishes a relationship between the average global strain in the composite and the local subcell strains. This relationship is known as a concentration tensor that can be used to predict the effective properties of the composite. Once the subcell strains are known, the average subcell stresses can be calculated using known subcell constitutive laws. Global stresses and strains are volume averages of subcell values.

In each subcell $(\alpha, \beta, \gamma)$ the average stress is defined as

$$
\bar{\sigma}^{(\alpha \beta \gamma)}=C^{(\alpha \beta \gamma)}\left[\bar{\epsilon}^{(\alpha \beta \gamma)}-\bar{\epsilon}^{I(\alpha \beta \gamma)}-\alpha^{(\alpha \beta \gamma)} \triangle T\right]
$$




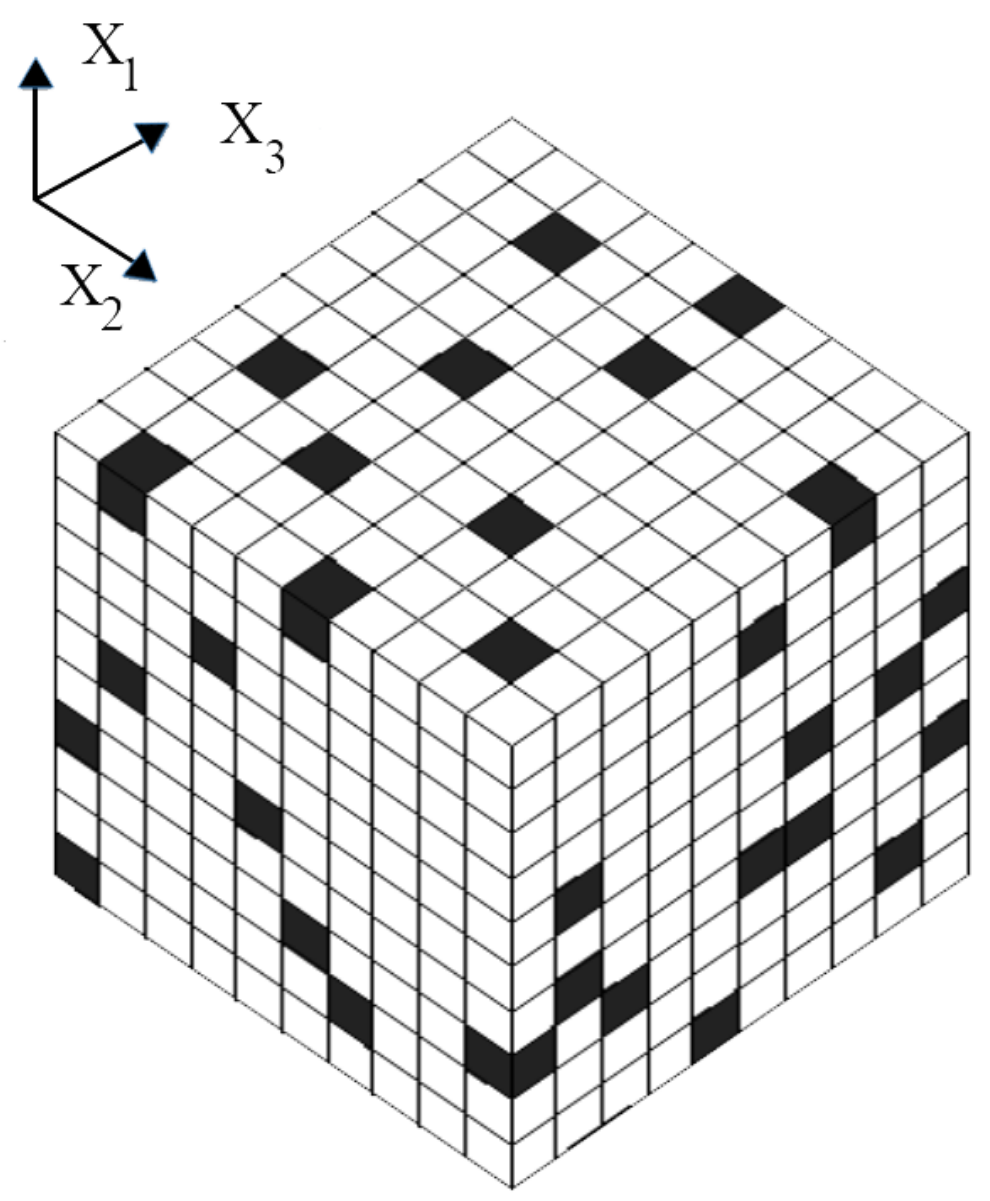

Figure 3.1: GMC's Repeating Unit Cell with white matrix subcells and black particle subcells.

Here, $C^{(\alpha \beta \gamma)}$ is the elastic stiffness tensor for the material in subcell $(\alpha, \beta, \gamma), \bar{\epsilon}^{(\alpha \beta \gamma)}$ is the average subcell strain vector, $\bar{\epsilon}^{I(\alpha \beta \gamma)}$ is the average subcell inelastic vector, and $\alpha^{(\alpha \beta \gamma)} \triangle T$ is the subcell thermal strain vector, $\bar{\epsilon}^{T(\alpha \beta \gamma)}$. Average strains in the composite are given by

$$
\bar{\epsilon}=\frac{1}{d h l} \sum_{\alpha=1}^{N_{\alpha}} \sum_{\beta=1}^{N_{\beta}} \sum_{\gamma=1}^{N_{\gamma}} d_{\alpha} h_{\beta} l_{\gamma} \bar{\epsilon}^{(\alpha \beta \gamma)}
$$

The average stress in the composite can be defined by

$$
\bar{\sigma}=\frac{1}{d h l} \sum_{\alpha=1}^{N_{\alpha}} \sum_{\beta=1}^{N_{\beta}} \sum_{\gamma=1}^{N_{\gamma}} d_{\alpha} h_{\beta} l_{\gamma} \bar{\sigma}^{(\alpha \beta \gamma)}
$$


where $d, h$, and $l$ are the dimensions of the total repeating unit cell, and $d_{\alpha}, h_{\beta}$, and $l_{\gamma}$ are the dimensions of subcell $(\alpha, \beta, \gamma)$.

The relationship between the global and subcell strains is defined by a system of equations that is constructed by requiring that displacements and tractions are continuous across unit cell and subcell boundaries. The displacement components $u_{i}$ at the interfaces within the unit cell and between unit cells must be continuous so that for $\alpha=1, \ldots N_{\alpha} ; \beta=1, \ldots N_{\beta} ; \gamma=1, \ldots N_{\gamma}$,

$$
\begin{aligned}
\left.u_{i}^{(\alpha \beta \gamma)}\right|_{\bar{x}_{1}^{(\alpha)}=d_{\alpha} / 2} & =\left.u_{i}^{(\hat{\alpha} \beta \gamma)}\right|_{\bar{x}_{1}^{(\hat{\alpha})}=d_{\hat{\alpha} / 2}} \\
\left.u_{i}^{(\alpha \beta \gamma)}\right|_{\bar{x}_{2}^{(\beta)}=h_{\beta} / 2} & =\left.u_{i}^{(\alpha \hat{\beta} \gamma)}\right|_{\bar{x}_{2}^{(\hat{\beta})}=h_{\hat{\beta}} / 2} \\
\left.u_{i}^{(\alpha \beta \gamma)}\right|_{\bar{x}_{3}^{(\gamma)}=l_{\gamma} / 2} & =\left.u_{i}^{(\alpha \beta \hat{\gamma})}\right|_{\bar{x}_{3}^{(\hat{\gamma})}=l_{\hat{\gamma}} / 2}
\end{aligned}
$$

enforcing the periodic boundary conditions assumed in the model, as

$$
\hat{\alpha}=\left\{\begin{array}{ll}
\alpha+1 & \text { when } \alpha<N_{\alpha} \\
1 & \text { when } \alpha=N_{\alpha}
\end{array} ; \hat{\beta}=\left\{\begin{array}{ll}
\beta+1 & \text { when } \beta<N_{\beta} \\
1 & \text { when } \beta=N_{\beta}
\end{array} ; \hat{\gamma}= \begin{cases}\gamma+1 & \text { when } \gamma<N_{\gamma} \\
1 & \text { when } \gamma=N_{\gamma}\end{cases}\right.\right.
$$

These condtions can be written in matrix form as

$$
\mathbf{A}_{G} \epsilon_{s}=\mathbf{J} \bar{\epsilon}
$$

where $\bar{\epsilon}=\left(\bar{\epsilon}_{11}, \bar{\epsilon}_{22}, \bar{\epsilon}_{33}, 2 \bar{\epsilon}_{23}, 2 \bar{\epsilon}_{13}, 2 \bar{\epsilon}_{12}\right)$ and $\epsilon_{s}=\left[\bar{\epsilon}^{(111)}, \ldots, \bar{\epsilon}^{\left(N_{\alpha} N_{\beta} N_{\gamma}\right)}\right]$.

Continuity of tractions requires

$$
\begin{aligned}
& \bar{\sigma}_{1 i}^{(\alpha \beta \gamma)}=\bar{\sigma}_{1 i}^{(\hat{\alpha} \beta \gamma)} \\
& \bar{\sigma}_{2 i}^{(\alpha \beta \gamma)}=\bar{\sigma}_{2 i}^{(\alpha \hat{\beta} \gamma)} \\
& \bar{\sigma}_{3 i}^{(\alpha \beta \gamma)}=\bar{\sigma}_{3 i}^{(\alpha \beta \hat{\gamma})}
\end{aligned}
$$


Using the constitutive law, Equation (3.1), these equations can be written in matrix form as

$$
\mathbf{A}_{M}\left(\epsilon_{s}-\epsilon_{s}^{I}-\epsilon_{s}^{T}\right)=0
$$

where $\mathbf{A}_{M}$ contains the elastic properties $C^{(\alpha \beta \gamma)}$ of the subcell material, $\epsilon_{s}^{I}=\left[\bar{\epsilon}^{I(111)}, \ldots, \bar{\epsilon}^{I\left(N_{\alpha} N_{\beta} N_{\gamma}\right)}\right]$ and $\epsilon_{s}^{T}=\left[\bar{\epsilon}^{T(111)}, \ldots, \bar{\epsilon}^{T\left(N_{\alpha} N_{\beta} N_{\gamma}\right)}\right]$.

Combining (3.8) and (3.6) gives

$$
\tilde{\mathbf{A}} \epsilon_{s}-\tilde{\mathbf{D}}\left(\epsilon_{s}^{I}+\epsilon_{s}^{T}\right)=\mathbf{K} \bar{\epsilon}
$$

where

$$
\tilde{\mathbf{A}}=\left[\begin{array}{c}
\mathbf{A}_{M} \\
\mathbf{A}_{G}
\end{array}\right], \quad \tilde{\mathbf{D}}=\left[\begin{array}{c}
\mathbf{A}_{M} \\
0
\end{array}\right], \quad \mathbf{K}=\left[\begin{array}{l}
0 \\
\mathbf{J}
\end{array}\right]
$$

For the elastic case considered here, Equation (3.9) becomes

$$
\tilde{\mathbf{A}} \epsilon_{s}=\mathbf{K} \bar{\epsilon}
$$

Solving for the subcell strains $\epsilon_{s}$ in terms of the global strains gives

$$
\epsilon_{s}=\mathbf{A} \bar{\epsilon}
$$

where $\mathbf{A}=\tilde{\mathbf{A}}^{-1} \mathbf{K}$. The mechanical concentration tensor $\mathbf{A}$ can be divided into submatrices as

$$
\mathbf{A}=\left[\begin{array}{c}
\mathbf{A}^{(111)} \\
\vdots \\
\mathbf{A}^{\left(N_{\alpha} N_{\beta} N_{\gamma}\right)}
\end{array}\right]
$$

Finally, we establish the effective elastic mechanical law of the composite

$$
\bar{\sigma}=\mathbf{B}^{*}(\bar{\epsilon})
$$


where the effective elastic stiffness tensor, $\mathbf{B}^{*}$, for the composite is given by

$$
\mathbf{B}^{*}=\frac{1}{d h l} \sum_{\alpha=1}^{N_{\alpha}} \sum_{\beta=1}^{N_{\beta}} \sum_{\gamma=1}^{N_{\gamma}} d_{\alpha} h_{\beta} l_{\gamma} C^{(\alpha \beta \gamma)} \mathbf{A}^{(\alpha \beta \gamma)}
$$

\subsection{Percolation Models}

The GMC unit cell, divided into cubic subcells, is a classic representation of a cellular material generated by a Voronoi tessellation. Tessellations are formal methods of subdividing a $d$-dimensional space into polyhedral cells. They can be built around a random or, in the case of GMC, a regular lattice. Lines are drawn from one site to all surrounding sites and the boundaries of the polyhedra (cubes) are formed by lines perpendicular to the site-site lines. GMC's unit cell with random cells designated as matrix or particle is also known as a random checkerboard, which is a simple version of a two-phase symmetric cell. It is symmetric because the material's morphology when phase one has a volume fraction of $\phi_{1}$ is statistically identical to the morphology of phase two when the volume fraction of phase one is $\left(1-\phi_{1}\right),[15]$. The random checkerboard is also potentially of a class of bi-continuous materials. These are materials that can simultaneously percolate in two phases. The random checkerboard can be used to model discrete percolation, where shared edges define connectivity or continuum percolation, where a potentially more complex nearest neighbor rule defines connectivity.

When an interface region is included in the model, GMC's cellular structure is closer to that of the interpenetrating particle model, or the permeable sphere model used in [40] or potentially the penetrable concentric shell, cherry pit model in [41]. Comparisons of percolation thresholds produced in this work with those developed analytically in these two models are difficult because in this work the interface volume fraction is kept separate from the particle volume fraction, whereas in the cited work the penetrable phase is considered part of the particle volume fraction. 


\subsection{Monte Carlo Simulations}

The repeating unit cell in GMC functions as an exact RVE for a material with a triply-periodic microstructure. In order to simulate random microstructures using GMC, this work assumes that GMC's RUC can approximate the RVE for a random composite. Although it is not possible to completely remove the influence of the periodic boundary conditions, if the RUC represents a large enough sample of the material microstructure, the periodic effects are minimized and a random microstructure is approximated. The characterization of the size of the RVE is based on the definition proposed in [42], as a sample of the material where the scale ratio $L / \delta \rightarrow \infty$, where $L$ is a characteristic dimension of the representative volume element, and $\delta$ is a characteristic dimension of the microstructure. For this three-dimensional model, the characteristic dimensions, $L$ and $\delta$, were taken to be the volumes of the RUC and a single particle, respectively. The three-phase GMC unit cell was constructed of $48 \times 48 \times 48$ square subcells. Each particle was modeled by a $2 \times 2 \times 2$ block of subcells. The interface was assumed to be half the width of the particle, or a surrounding layer one subcell in thickness. For the two-phase model (particle and matrix), the unit cell measured $24 \times 24 \times 24$ with particles $1 \times 1 \times 1$. This resulted in equivalent scale ratios, $L / \delta=\left(48^{3} / 2^{3}\right)=\left(24^{3} / 1^{3}\right)=13,824$ for each model.

The major focus of this work is the model's ability to capture a sharp increase in mechanical properties associated with the mechanisms of percolation. The dimensions for the three-phase model (particle, interface, and matrix) were chosen as those for which the next larger size resulted in no significant differences in graphs of the average properties based on the same number of simulation runs. This same size ratio was then used for the two-phase model. A similar criteria was used to choose the number of simulations.

Particle positions were assigned with a uniform random distribution and $300 \mathrm{mi}-$

crostructural realizations were performed for each volume fraction. Elastic stiffness 
tensors were calculated for each microstructure. In general, the simulated composites would be expected to be statistically isotropic however, where microstructural arrangements play a significant role, it was at the expense of isotropy. To aid in distinguishing these effects, results are presented in terms of elastic constants and elements of the elastic stiffness tensor for an orthotropic composite; $E_{11}$ designated as the longitudinal modulus, and $E_{22}$ and $E_{33}$, as axial moduli, are used in the graphical discussion. The normal; $C_{11}, C_{22}, C_{33}$; off-diagonal, $C_{12}, C_{13}, C_{23}$; and shear components, $C_{44}, C_{55}, C_{55}$; of the elastic stiffness matrix, C, relating stress $\sigma$ to elastic strain $\epsilon$, through

$$
\sigma=\mathrm{C} \epsilon
$$

are used to distinguish the effects of microstructure on effective composite properties. In common with other micromechanics models, for example, the well-known MoriTanaka method, [31, 32], GMC includes no shear normal coupling and therefore the greatest degree of anisotropy it can capture is that of an orthotropic material. Differences between degrees of anisotropy were often difficult to assess, therefore, in this work, when the material could be characterized by three constants, it was labeled isotropic, but additional increases in anisotropy were only specified by the number of distinct constants in the stiffness tensor. 


\section{Chapter 4}

\section{Mechanical Percolation in Two-Phase \\ Composite; Cubic Particles}

The model composite consists of metallic nanocubes with an aspect ratio, $\alpha=1$. The

properties and geometry of this included phase were suggested by the gold nanocubes made by Murphy et al. [43, 44]. The potential exists for the cubes, initially grown while suspended in water, to be either dispersed in a liquid polymer and cured in place, or applied to a surface and spin-coated with a polymer thin film. Recent work by Niklaus and Shea [23] investigated electrical and mechanical percolation of metal-ion implantation in just this type of material system.

In this work, the metallic cubes and polymeric matrix were assigned isotropic elastic properties. The metal particles were assigned a stiffness on the order of $10^{10}$ $\mathrm{Pa}$, and a Poisson's ratio of 0.33 . The polymer was assigned a stiffness of $\sim 10^{4}$ $\mathrm{Pa}$ and a Poisson's ratio of 0.45 . This composite would be similar to composites using PDMS (polydimethylsiloxane) [23], suggested as a good candidate for thin film, flexible sensing devices, [23, 45].

Random microstructures for the two-phase, matrix and particle, composite were simulated at volume fractions from 0 to 1 in steps of 0.05 ; 300 microstructural realizations were generated for each volume fraction. GMC was used to approximate the effective elastic properties associated with each microstructure. Figure 4.1 plots the normalized minimum, average, and maximum values of the axial elastic stiffness, $E_{11} / E_{\text {matrix }}$, at each volume fraction. 


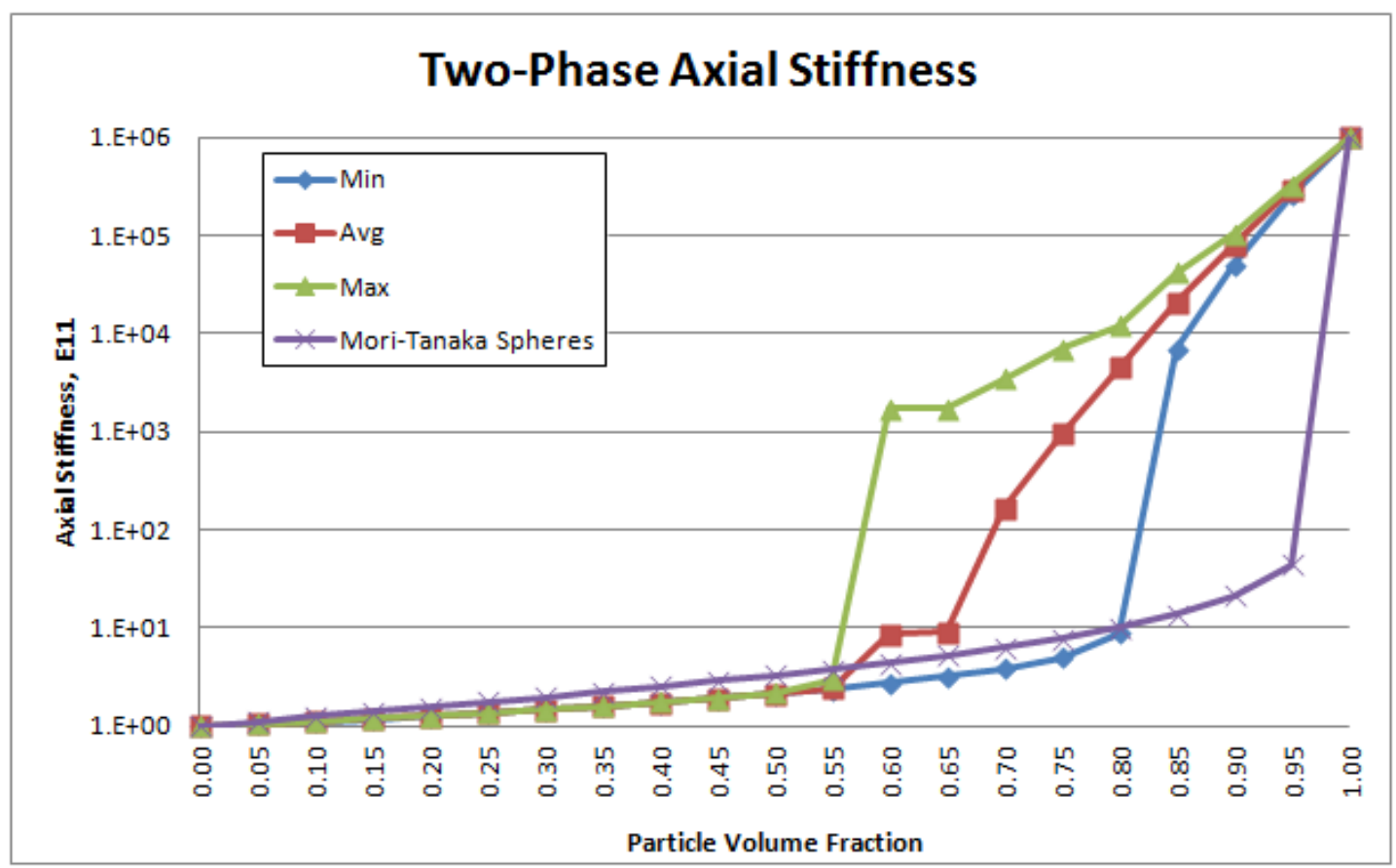

Figure 4.1: Effective elastic axial stiffnesses for the simulated two-phase composite; normalized minimum, average, and maximum values. Simulations based on random microstructure are compared to the Mori-Tanaka micromechanics model.

Plots of the remaining two axial moduli are similar to fig. 4.1; neither the shear moduli nor the Poisson's ratios showed any variation. The simulated values are also compared to predictions from the Mori-Tanaka (MT) mean-field model for a composite with a random arrangement of spheres. The differences between the simulated results and the MT predictions, at the lower volume fractions, are similar to those reported in [34] for high aspect ratio rods. They are largely due to the assumed geometry (cubes versus spheres) and underlying assumptions of each model (unit cell versus mean-field). Since Mori-Tanaka does not incorporate a specific microstructural arrangement, its predictions show no variability.

Figure 4.1 suggests that the first volume fraction where microstructural effects can make a contribution is 0.60 , where the maximum value curve takes its largest jump. Figure 4.2 shows distributions of the axial modulus for volume fractions of 0.6 through 0.85 . The mean values change with volume fraction, in order to compare 
distributions, zero mean fields, $f(v)$, were constructed,

$$
f(v)=\frac{E^{*}}{E_{\text {matrix }}}-1
$$

and plotted.

The distributions of moduli at both 0.60 and 0.65 are essentially binary. In each case, the simulation produced one microstructure with a relative modulus $E_{11} / E_{m}$ $\simeq 10^{3}$; the remaining 299 are tightly clustered around a single, essentially modal value, see figs. $4.2 \mathrm{a}, 4.2 \mathrm{~b}$. At volume fractions of 0.7 and 0.75 , the mean value curve takes its largest jump. The distributions of moduli are still relatively disjoint. The majority of microstructures have moduli corresponding to a mean/modal value. As volume fraction increases, a greater number of microstructures produce distinct higher and lower moduli, figs. 4.2c, 4.2d. Between 0.80 and 0.85 , the minimum value curve takes its largest jump. The distribution of moduli for 0.8 are more balanced about the mean, but still occur at widely spaced intervals, fig. 4.2e. At 0.85, in contrast to the lower volume fractions, the distributions are more continuous, fig. $4.2 f$.

In general, the simulated microstructures should be statistically isotropic, with the ratio of the longitudinal to transverse modulus, $E_{11} / E_{22}=1$; this is not consistently true for the simulated values between 0.60 - 0.85. Neither is the isotropic relationship between the three elastic constants, $E=2 G(1+\nu)$ true, on average, except at the very low and high volume fractions.

Three elements define the effective stiffness of the composite; the deterministically controlled volume fraction, the contrast ratio between the phases and the microstructure. Globally, at volume fractions $\leq 0.55$, there is little variation in the predicted properties suggesting that microstructural effects are minimal. Particles in this range are widely spaced and the likelihood of particle interaction or clustering is low. The matrix forms a fully connected phase. 

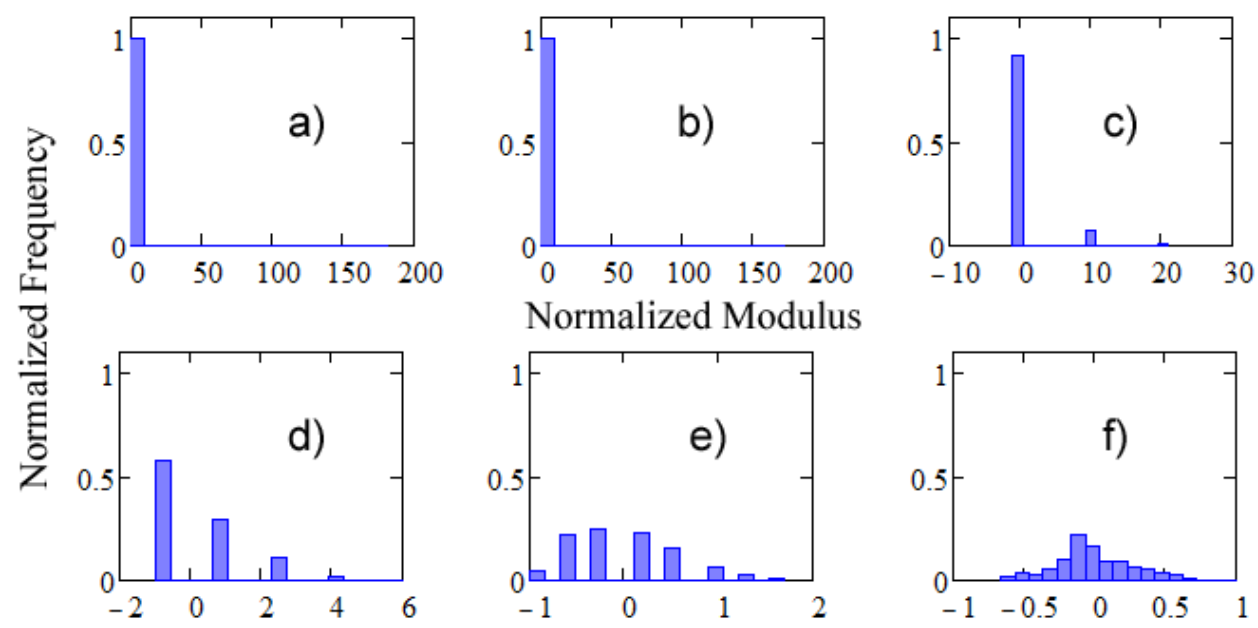

Figure 4.2: Distributions of axial moduli in the two-phase composite at various volume fractions: a) 0.60 b) 0.65 c) 0.70 d) 0.75 e) 0.80 f) 0.85 .

Above a volume fraction of 0.55 , microstructural effects are increasingly significant. Stiffness tensors for the minimum valued microstructures are fairly consistently isotropic. Maximum valued microstructures, however, tend to be transversely isotropic, up to 0.75 , and require 5-7 constants for higher volume fractions. The loss of isotropy is initially due to emerging distinctions between the normal components, i.e., $C_{11} \neq C_{22}, \neq C_{33}$, but eventually includes the off-diagonal elements, $C_{12} \neq C_{13}$ $\neq C_{23}$. The shear components showed little variation, generally $C_{44}=C_{55}=C_{66}$.

This type of shift in symmetries, associated with changes in the axial stiffnesses, suggests a change in the effective aspect ratio of the particles, i.e the formation of particle stacks. An analysis of axial sections of sample RUCs established that there was one continuous fiber stack, from top to bottom, in the longintudinal direction, of the RUC in each of the maximum moduli microstructures at 0.60 and 0.65 , no continuous stack was present in representative average and minimum valued microstructures at these volume fractions. In a periodic construction, this constitutes the reinforcement of a continuous fiber. For volume fractions of 0.70 and 0.75 , the same effects appear to be in play, however for each of these volume fractions, more than one 'large' 
axial modulus microstructure was produced. The RUCs for 0.75 showed that the sample microstructures with the two largest moduli had 3 continuous particle stacks, the sample microstructure with the next largest contained 2 continuous stacks and the microstructure with the third largest, one stack. Thus, there is a clear correspondence between the number of stacks and the magnitude of the axial modulus. Additional geometric effects., e.g. transverse clustering resulting in 'wider' stacks, and the production of longer, but perhaps not continuous, particle stacks are likely to cause additional fluctuations in the moduli, and result in cases where the same number of continuous stacks produce different moduli.

At the lower volume fractions, where the composite still has a significant volume of a compliant matrix, the presence of a continuous particle stack, in the axial direction, has a significant effect on the overall axial modulus. As the volume fraction increases, the probability of connected microstructural elements also increases. However, this mechanism of continuous reinforcement also becomes more equally likely in all three coordinate directions. Combined with the additional composite stiffening due to the increase in particle volume fraction, the overall microstructural effect is less dramatic. As the volume fraction reaches 0.9 and higher, the particle phase is continuous and variations in the positions of isolated regions of the compliant matrix do not significantly affect composite properties.

Based on the simulations, predictions of an apparent percolation threshold, visible as the first volume fraction where the particle phase forms a connected, edge to edge, microstructure, is 0.60 ; the probability of this occurring, based on the simulations, is relatively low. Using a percolation threshold value of $\phi_{c}=0.60$, the two percolation models, one based on eqn. (2.3), using $a=0.65, f=3.75$, and $\phi_{c}=0.60$, and the second on eqns. $(2.3,2.4)$ with $b=6.0$ were fit to the data and are shown in fig.4.3. The first model, the power law, captures early trends in the mean values, but is less accurate at the higher volume fractions; the second model, the series parallel model, 
which is only defined for $v \geq \phi_{c}$, fits the higher volume fractions well with a large percolation exponent.

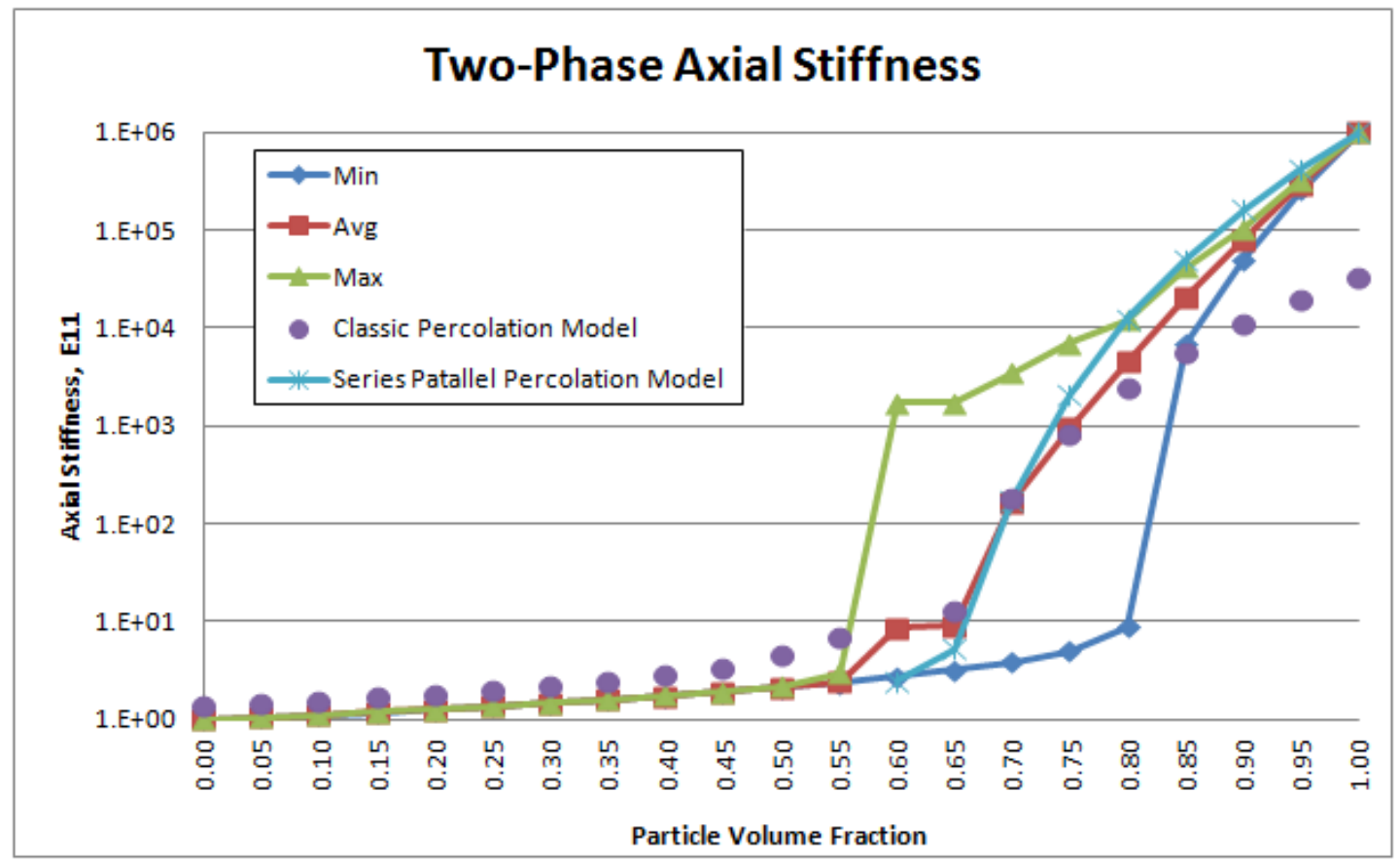

Figure 4.3: Comparison of simulated effective elastic axial stiffness for two-phase composite; normalized minimum, average, and maximum values, to classic percolation model based on eqn.(2.3), with $\phi_{c}=0.60, a=0.65$, and $f=3.75$; and series parallel model based on eqn. $(2.3,2.4)$, with $b=6.0$

The true percolation threshold, the volume fraction beyond which all of the simulated microstructures contain a connected microstructure, is closer to 0.80 . This can be seen in fig. 4.4, as the percent of simulated microstructures, out of 300 , where a continuous stack occurs.

It is important to remember that the model assumes perfect bonding between the phases. This is a hopeful expectation between particle and matrix, but probably not a realistic one between particle and particle. This means that, unlike electrical conductivity where contact produces the percolation effect, it can really only be assumed that the modulus in compression would be enhanced by a continuous stack. If the particles stacks were additionally constrained from separating, perhaps by 
the matrix microstructure, then assumptions about an enhanced modulus in tension would be more valid.

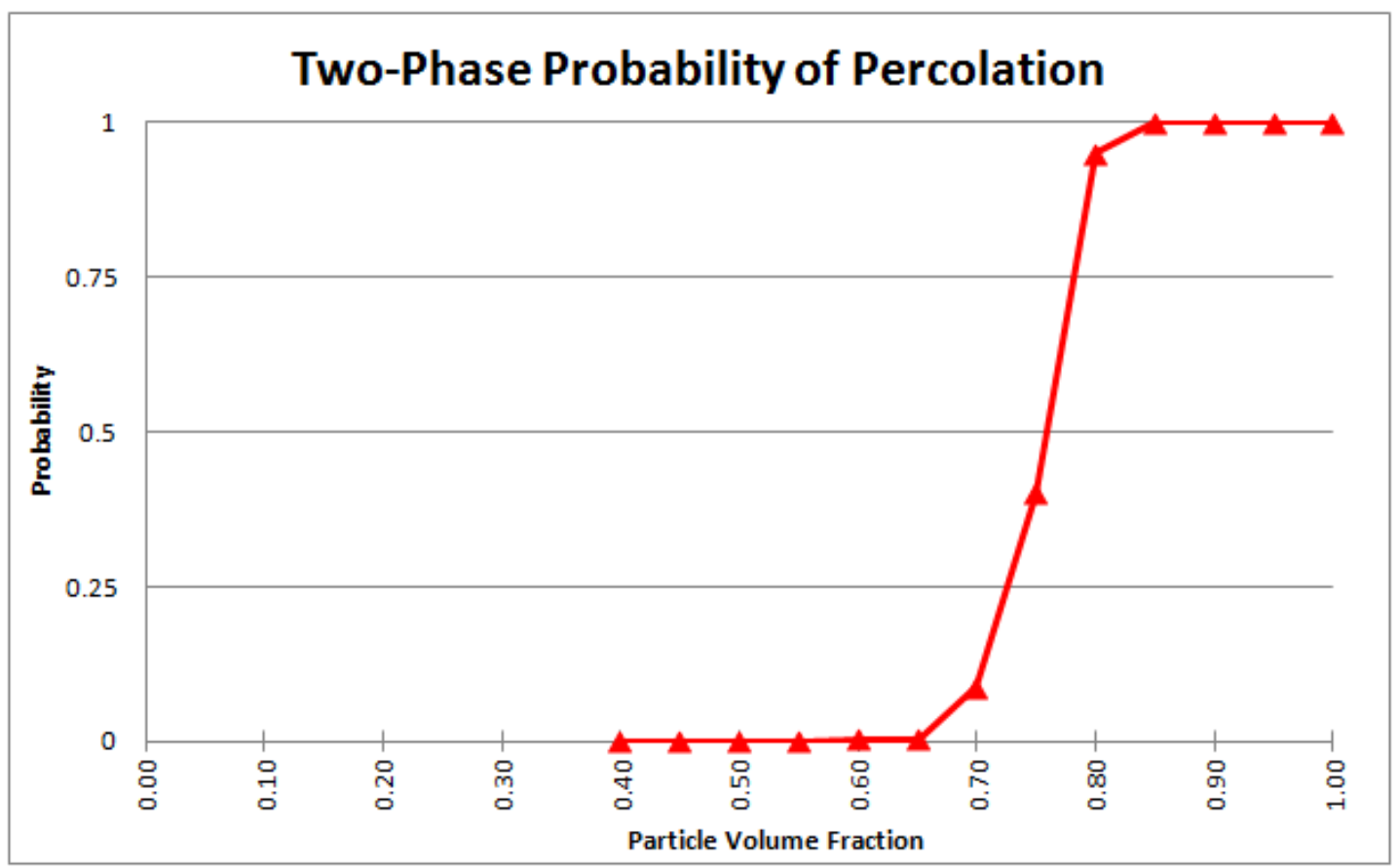

Figure 4.4: Percent of simulated microstructures in the two-phase composite that have continuous stacks. 


\section{CHAPTER 5}

\section{Mechanical Percolation in Three-Phase \\ Composite; Cubic Particles}

A second composite model was also studied. In this case, the composite was modeled as a three-phase nanocomposite consisting of particles, interface, and matrix. The nanoscale is introduced into the nanocomposite model by linking the interface thickness to the dimension of the particle. The properties of the particles and matrix were the same as the two-phase composite. The interface region was assigned a stiffness

equal to the geometric mean of the polymer and particles, $\sqrt{E_{m} E_{p}}$, and a Poisson's ratio of 0.45 .

Figure 5.1 shows the normalized minimum, average, and maximum effective axial stiffnesses for the three-phase composite; 300 microstructural realizations were generated for each volume fraction from 0 to 1 in steps of 0.05 . Two regions show variability; regime 1 , between 0.05 and 0.40 , and regime 2 , between 0.60 and 0.90 .

In regime 2 , the three-phase, composite has only two remaining phases, interface and particles, so its effective contrast ratio is $\gamma=\sqrt{E_{p} E_{I F}}=10^{3}$, the same as for the two-phase material; the trends and underlying mechanisms are the same.

More interesting is the variability in the low volume fraction range of regime 1. In generating the random microstructures, the interface was simulated as an effect of particle placement, rather than as a distinct third phase. Particle subcell positions were established first and then interface subcells were inserted to replace any matrix subcells surrounding the particle. As a result, if two particles were placed in adjacent 


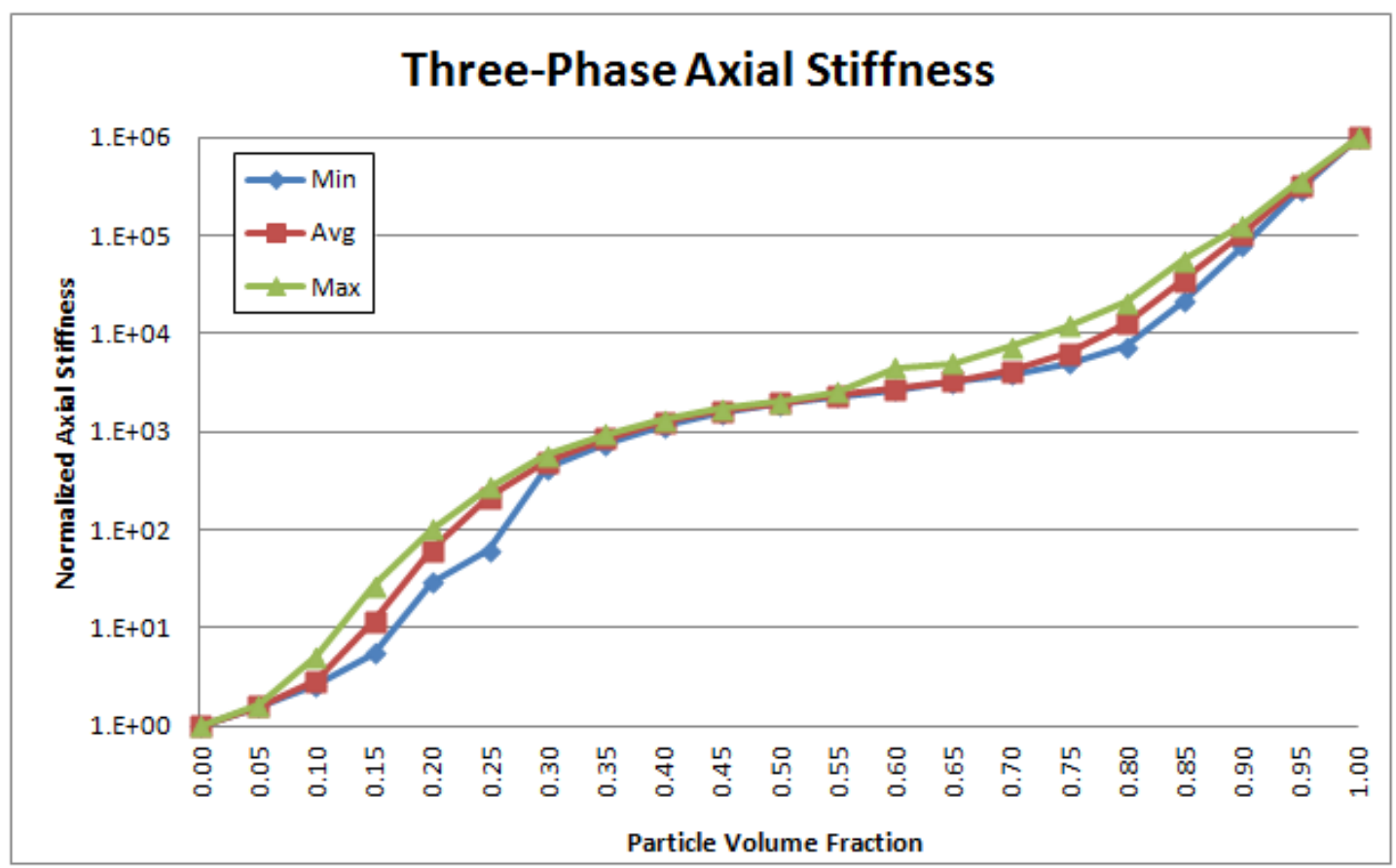

Figure 5.1: Simulated effective elastic stiffness for three-phase composite; normalized minimum, average, and maximum values.

subcells, no interface was inserted between them.

When the particles are widely dispersed, each $2 \times 2 \times 2$ block of subcells, one particle, can be surrounded by fifty-six interface subcells. If good dispersion is maintained, then all of the matrix subcells will have been replaced by interface subcells at a volume fraction of 0.125 . Random placement, resulting in particle clustering, delays this effect. Figure 5.2 compares a theoretical, or ideal dispersion, to simulated average, matrix and interface volume fractions. It shows that matrix subcells, on average, persist to a volume fraction of 0.40 ; in the minimum valued microstructures, matrix material may be present up to 0.60. At a volume fraction of $\sim 0.10$, the simulated matrix and interface volume fractions are equal; at $\sim 0.20$, simulated matrix volume fraction equals particle volume fraction. A maximum volume fraction of interface subcells occurs at $\sim 0.25$; subsequent particles replace more interface subcells than they produce.

There is also variability in the shear modulus, fig. 5.3. This is localized in the 


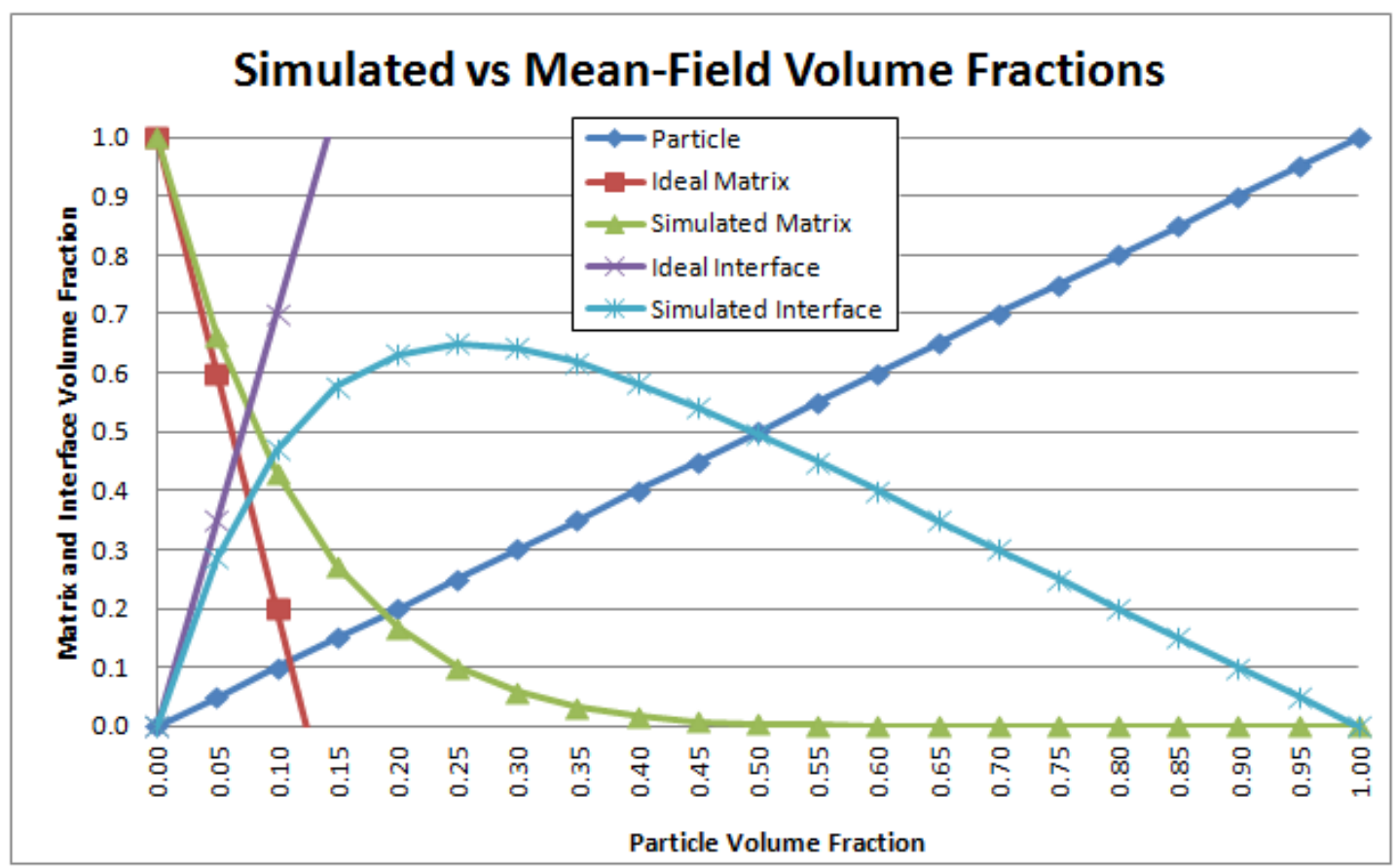

Figure 5.2: Matrix and interface volume fraction versus particle volume fraction; simulation values and ideal predictions.

region where the matrix volume fraction first vanishes in the maximum valued microstructures, $\sim 0.40$, and continues until the final remaining matrix subcells in the minimum valued microstructure, $\sim 0.60$, are gone. This effect was not present in predictions based on the two-phase model.

In contrast to the two-phase model, the three-phase composite simulations show two regions where random variations in the microstructure affect the elastic properties. Variability in the high range of volume fractions, $>0.55$, is well described by the mechanisms of two-phase percolation. Contrast ratio affects the magnitudes of the predicted moduli, but not the onset of percolation.

In regime 1 of the three-phase composite, material symmetries are difficult to establish. In many cases, estimations of distinctly different elements become subjective when the magnitudes of the differences are not large. Minimum valued microstructures were isotropic at 0.05 , and essentially transversely isotropic for volume fractions from 0.10 to 0.40 ; the plane of isotropy varying with microstructure. At volume frac- 


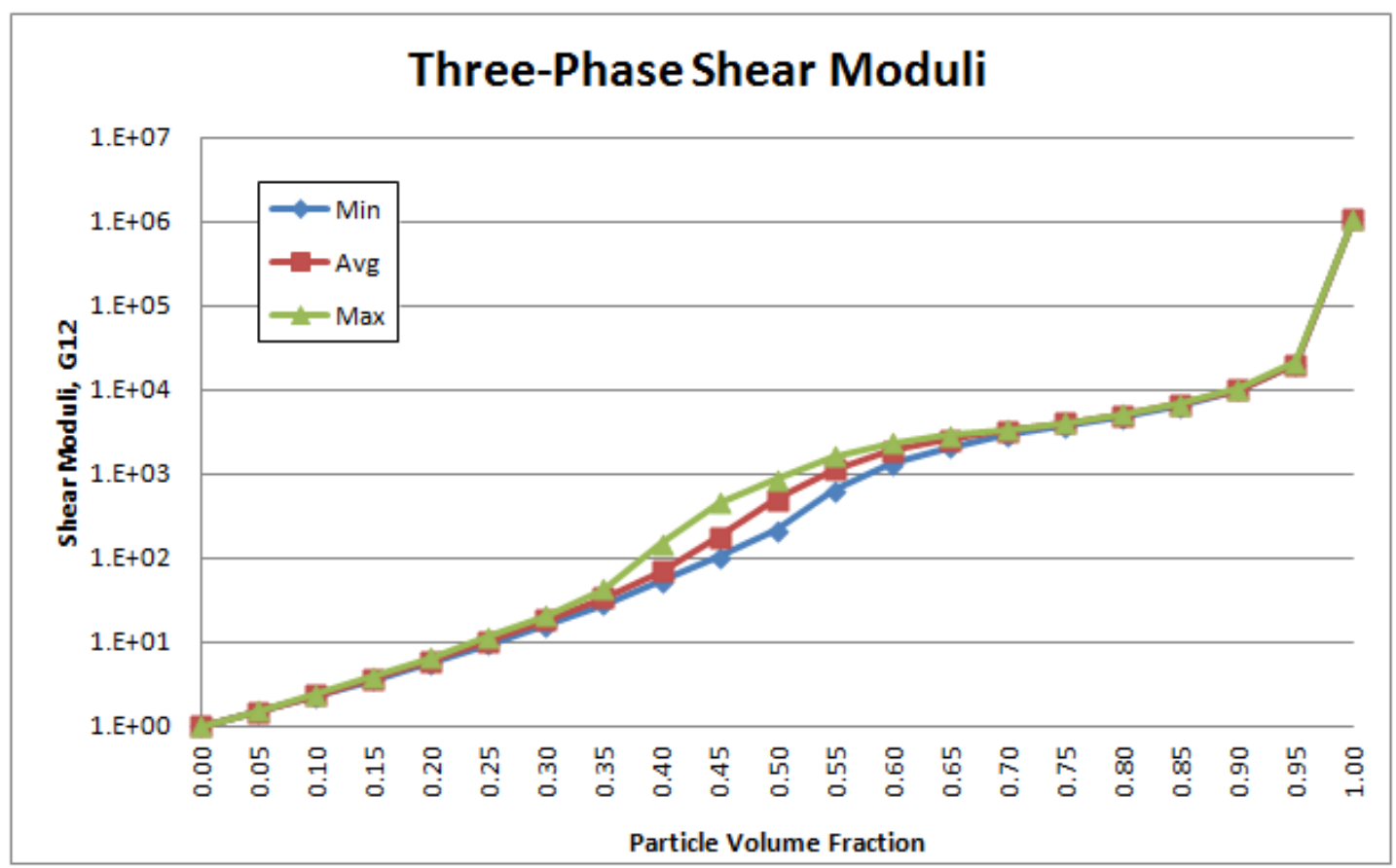

Figure 5.3: Distributions of shear moduli in the three-phase composite. In comparison, there is no variation in the shear modulus in the two-phase composite.

tions of $0.10,0.15$, and 0.20 , the maximum valued microstructures had 4 distinct constants, between 0.25 and $0.40,6$ or 7 constants were required.

Continuous particle stacks do not occur until volume fractions above 0.6 ; similar to the two-phase composite. Edge-to-edge stacks composed of both particles and interface, with no separating matrix material, first occur at 0.10 , and are the defining microstructural difference between minimum and maximum moduli up to 0.20. From 0.25 to 0.40 , the number of stacks were similar in minimum, average, and maximum valued microstructures. Above 0.40, where matrix material has largely been eliminated, particle-interface assemblies represent the bulk of the microstructure. Stack composition, $\sim 25 \%$ particles per stack, did not change significantly through the range 0.10 to 0.25 .

Variation in the lower range of volume fractions is the result of a number of competing mechanisms; the simple increase in the volume fraction of the particles, the 'replacement' of matrix material by the stiffer interface material, and the formation 
of a pseudo-percolated microstructure consisting of particle-interface stacks. Based on the volume fraction curves, an apparent percolation threshold for the three-phase material can be defined at the first occurrence of an edge-to-edge, particle-interface stack, at about 0.10 . This offers a better representation of experimentally observed values for nanocomposites, than the two-phase model. The two empirical percolation models were not good fits to the pseudo-percolation in this range, the simulated curves were consistently too flat for the power-law based models. Changes in the stiffness of the interface region, and its assumed thickness, would be expected to affect the early steepness of the curve and the apparent percolation threshold.

In the three-phase model, the assumptions about perfect bonding are less of a problem because none of the effects depend solely on particle particle bonds. It is again assumed that the interface, since it was originally matrix material, has a good bond to the particles. However, a more accurate conclusion, based on the model results, would be that the compressive modulus would be enhanced by the microstructure. 


\section{Chapter 6}

\section{EFFECt of Interfacial Stiffness AND Thickness}

Three groups of simulations were completed varying the interface region elastic stiffness. Each group used the same particle and matrix properties and structure as the three-phase composite in Chapter 5. The interface region for each simulation group also had a Poisson's ratio of $0.45 ; 300$ microstructural realizations were again generated for each volume fraction from 0 to 1 in steps of 0.05 . All graphical data in this chapter has been normalized by the matrix stiffness.

In the first group, the interface region was assigned a stiffness on the order of $10^{6} \mathrm{~Pa}$, creating a material region more compliant than in the original three-phase simulations. This group will be denoted as having a 'compliant' interface. The second group was assigned a stiffness for the interface equal to the geometric mean of the

polymer and particles, exactly the same as the three-phase composite, previously discussed, with a magnitude on the order of $10^{7} \mathrm{~Pa}$. This group will be referred to as having an 'original stiffness'. Finally, the third group had an interface stiffness on the order of $10^{9} \mathrm{~Pa}$, creating a stiffer material than the original group. Following the pattern, this group will be referred to as having a 'stiff' interface.

Three variations of the interface thickness surrounding each particle were investigated for each simulation group; compliant, original stiffness, and stiff. The first variation used an interface thickness equal to $1 / 3$ the particle diameter, creating an interface region thinner than the original composite. This will be referred to as the 'thin' interface. The second variation used the original dimensional thickness, similar to the three-phase composite, where the interface is equal to $1 / 2$ the particle 
diameter. This will be denoted the 'original thickness'. Finally, the third variation in each group used an interface thickness equal to the particle diameter, thus creating a region thicker than that in the original composite. This will be referred to as the 'thick' interface.

\subsection{Stiffness VARIATIONS}

First, graphs are presented of the three variations of each of the three groups, for a total of nine cases, which show the effect of thickness on compliant, original, and stiff interfaces. Figure 5.1 shows the results from Chapter 5 and will be used as a baseline for the results in this chapter. This three-phase composite has an original interface stiffness and an original interface thickness and is designated as [original stiffness, original thickness].

The first group of simulations illustrate the effect of thickness on the a compliant interface. In fig. 6.1 top, the normalized minimum, average, and maximum effective elastic axial stiffnesses for the [Compliant, Thin] composite are shown; in fig. 6.1 middle, [Compliant, Original Thickness]; and in fig. 6.1 bottom, [Compliant, Thick].

The second group of simulations illustrate the effect of thickness on the original stiffness interface. In fig. 6.2 top, the normailzed minimum, average, and maximum

effective elastic axial stiffnesses for the [Original Stiffness, Thin] composite are shown; in fig. 6.2 middle, [Original Stiffness, Original Thickness]; and in fig. 6.2 bottom, [Original Stiffness, Thick].

The third group of simulations illustrate the effect of thickness on a stiff interface. In fig. 6.3 top, the normalized minimum, average, and maximum effective elastic axial stiffnesses for the [Stiff, Thin] composite are shown; in fig. 6.3 middle, [Stiff, Original Thickness]; and in fig. 6.3 bottom, [Stiff, Thick]. 

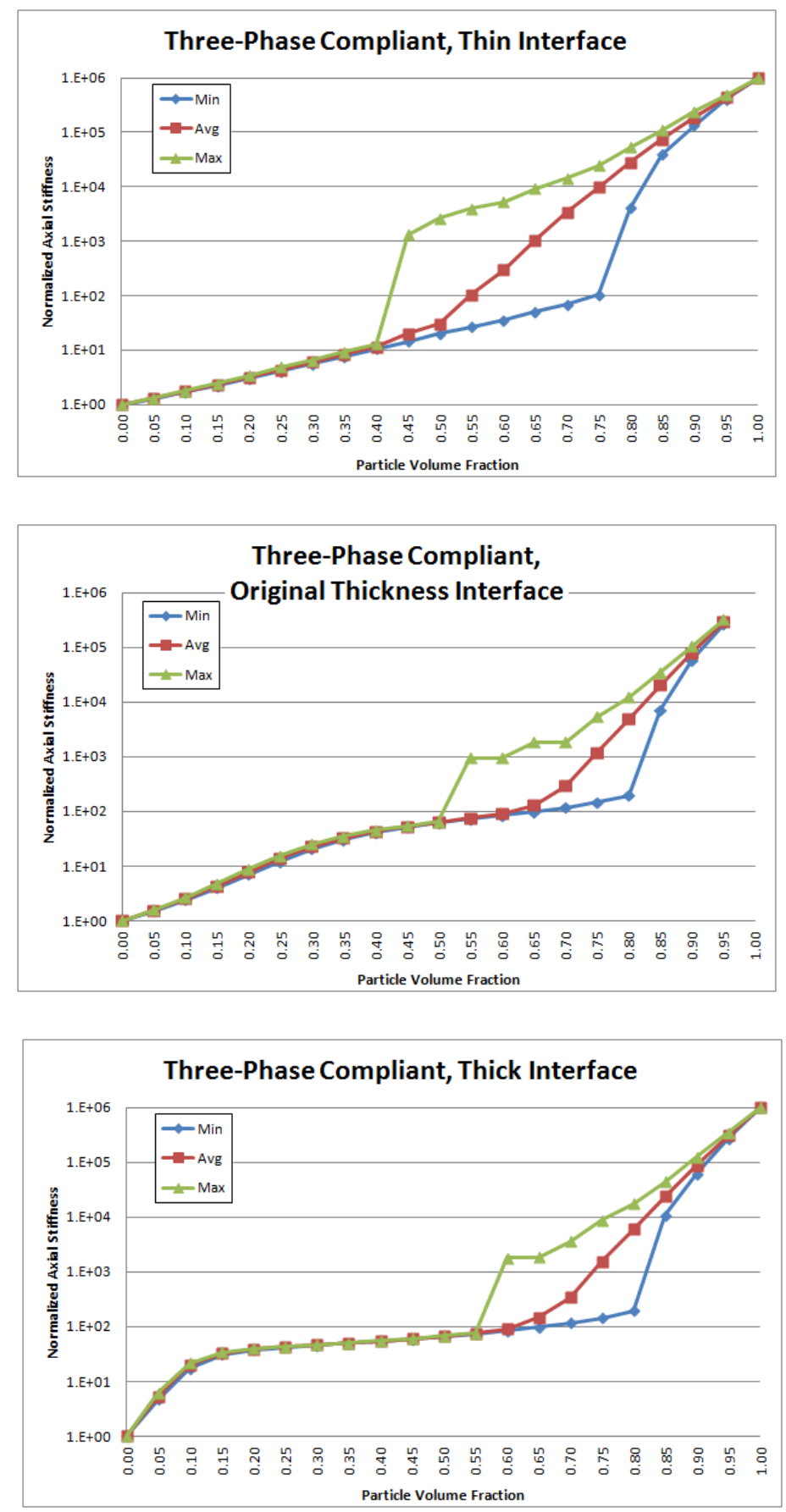

Figure 6.1: Simulated effective elastic axial stiffness (normalized minimum, average, and maximum values) for top: [Compliant, Thin]; middle: [Compliant, Original Thickness]; and bottom: [Compliant, Thick] 

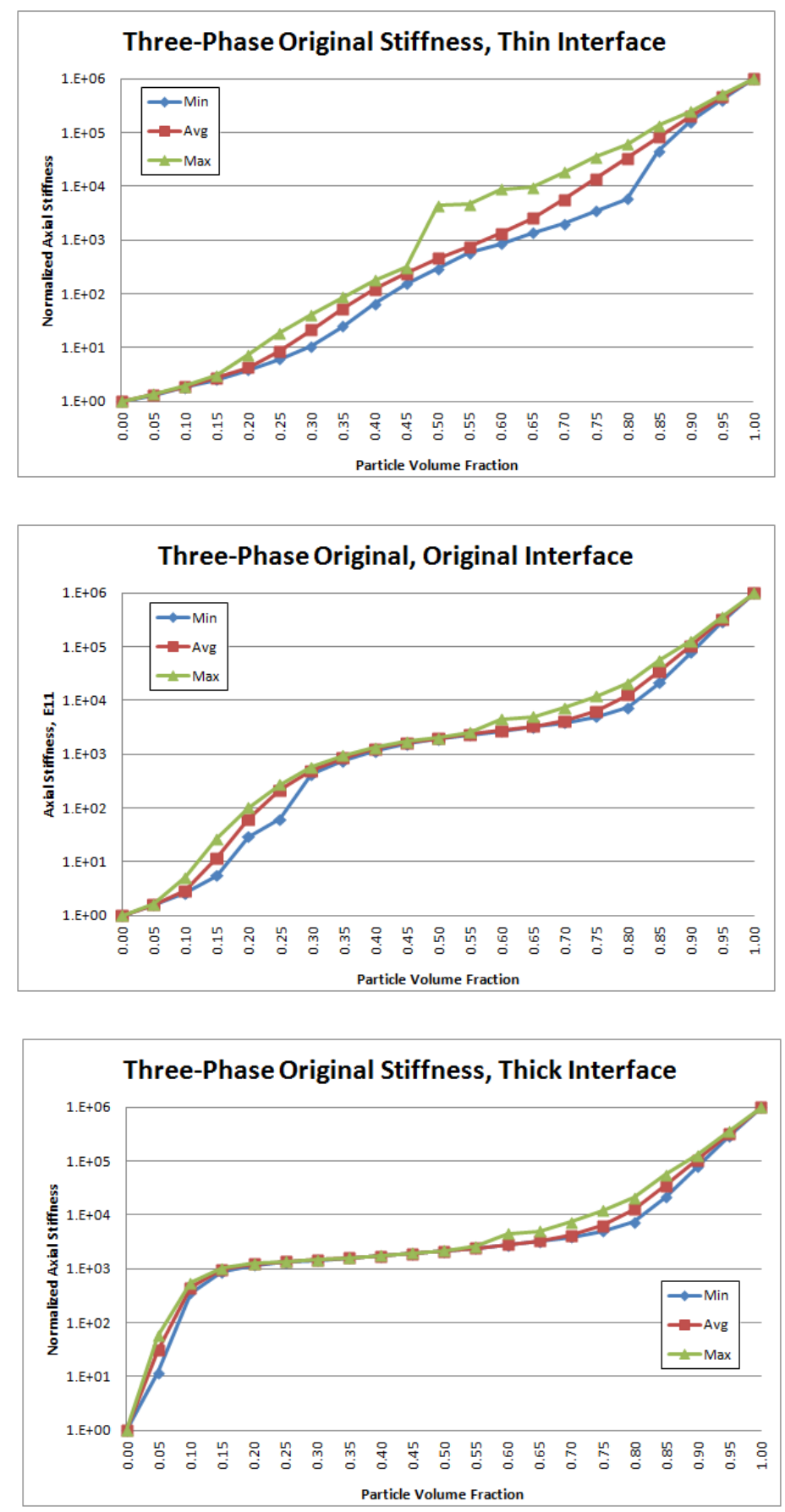

Figure 6.2: Simulated effective elastic axial stiffness (normalized minimum, average, and maximum values) for top: [Original Stiffness, Thin]; middle: [Original Stiffness, Original Thickness]; and bottom: [Original Stiffness, Thick] 

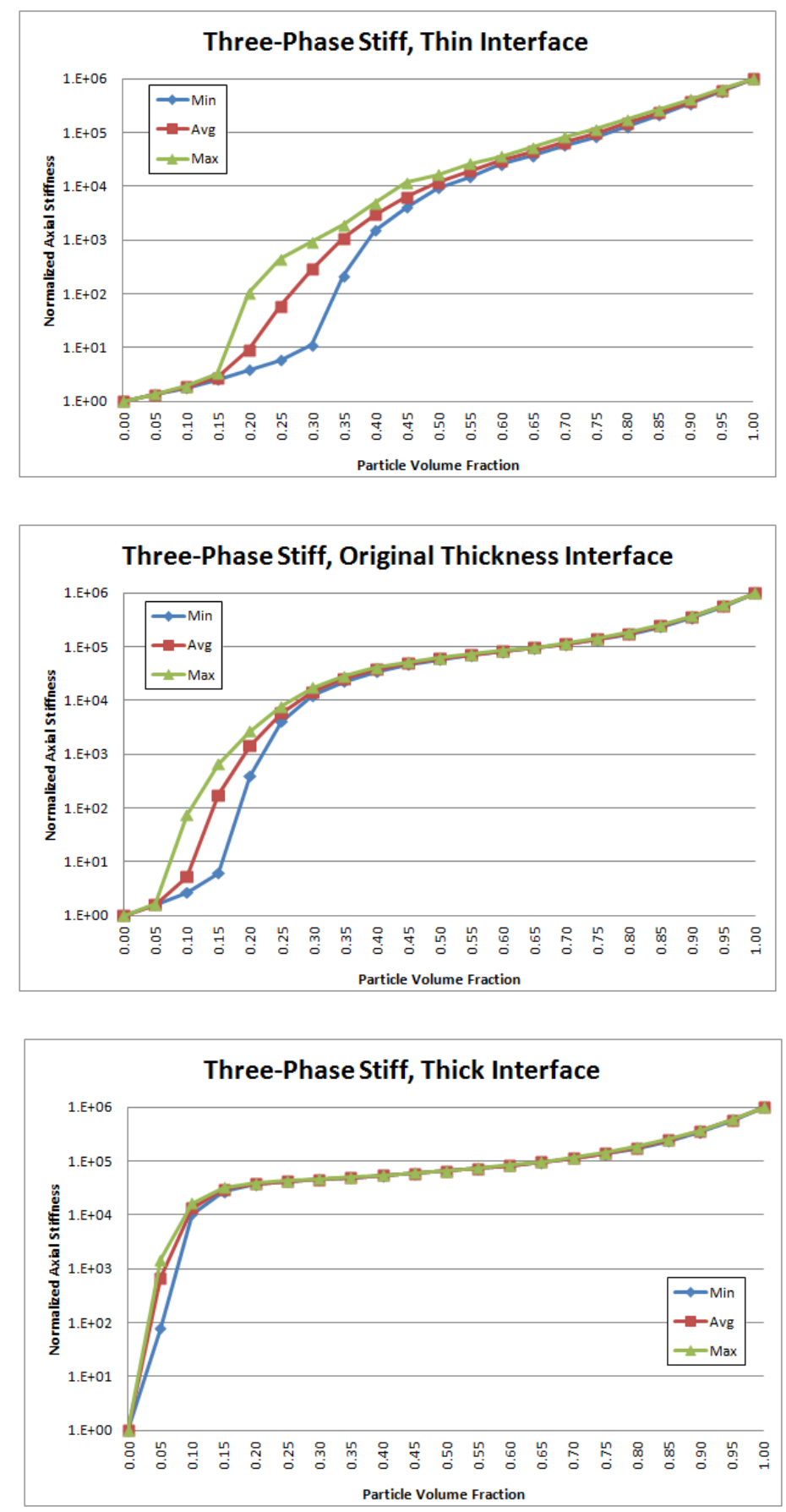

Figure 6.3: Simulated effective elastic axial stiffness (normalized minimum, average, and maximum values) for top: [Stiff, Thin]; middle: [Stiff, Original Thickness]; and bottom: [Stiff, Thick] 


\subsection{Thickness Variations}

The following results reorganize the previously presented data. Instead of graphing by groups of stiffness with variations in thickness, these following graphs will group thickness with variations in stiffness. Three groups of simulations were completed varying the interface region thickness. First, graphs are presented each containing three variations of the three groups, for a total of nine cases, which show the effect of stiffness on the thin, original, and thick interfaces.

The first group of simulations are those with a thin interface, and varying stiffnesses. In fig. 6.4 top, the normalized minimum, average, and maximum effective elastic axial stiffnesses for the [Thin, Compliant] composite are shown; in fig. 6.4 middle, [Thin, Original Stiffness]; and in fig. 6.4 bottom, [Thin, Stiff].

The second group of simulations are those with the original interface thickness and varying stiffnesses. In fig. 6.5 top, the normalized minimum, average, and maximum effective elastic axial stiffnesses for the [Original Thickness, Compliant] composite are shown; in fig. 6.5 middle, [Original Thickness, Original Stiffness]; and in fig. 6.5 bottom, [Original Thickness, Stiff].

The third group of simulations are those with a thick interface, and varying stiffness. In fig. 6.6 top, the normalized minimum, average, and maximum effective elastic axial stiffnesses for the [Thick, Compliant] composite are shown; in fig. 6.6 middle, [Thick, Original Stiffness]; and in fig. 6.6 bottom, [Thick, Stiff]. 

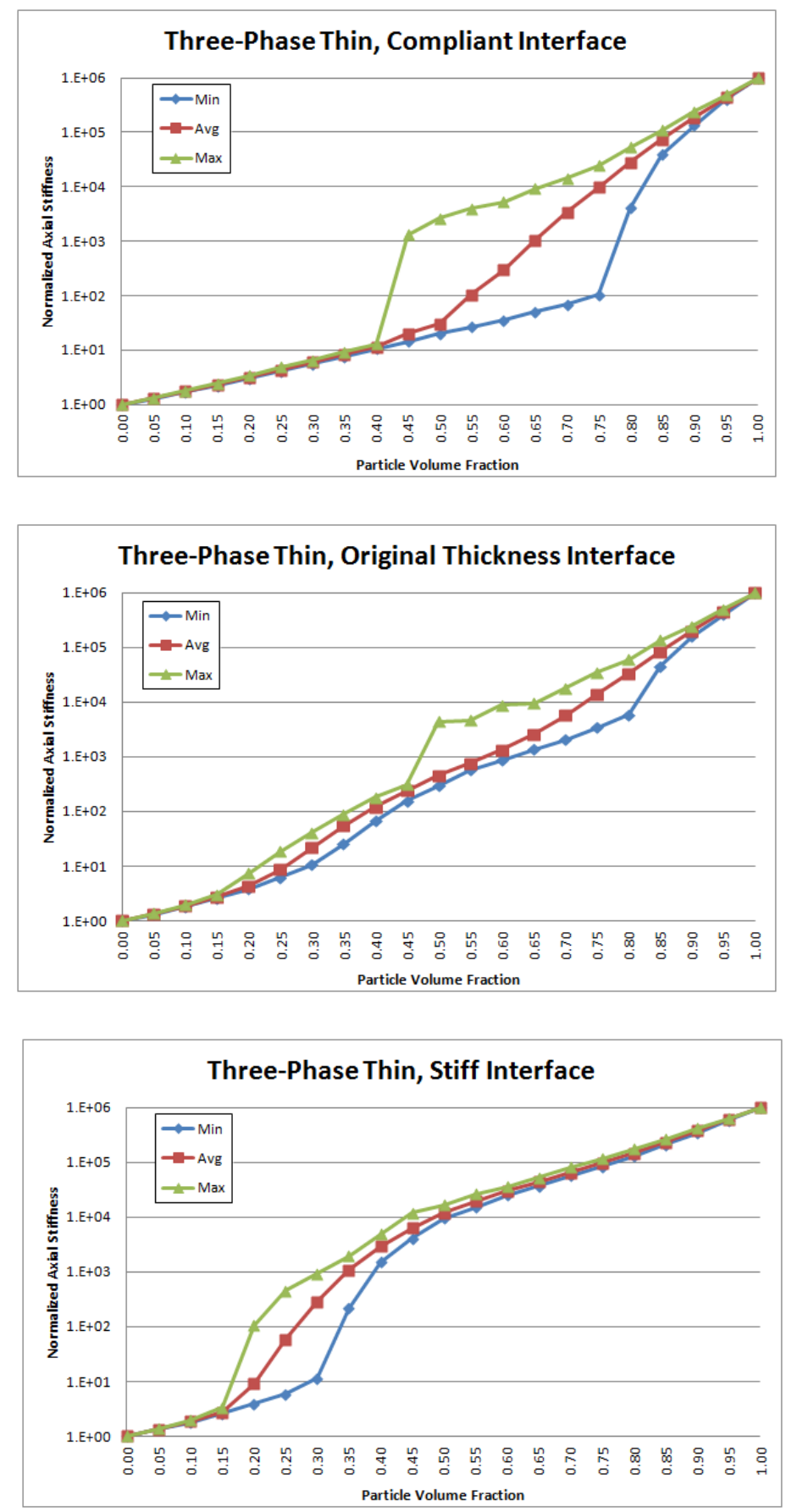

Figure 6.4: Simulated effective elastic axial stiffness (normalized minimum, average, and maximum values) for top: [Thin, Compliant]; middle: [Thin, Original Stiffness]; and bottom: [Thin, Stiff] 

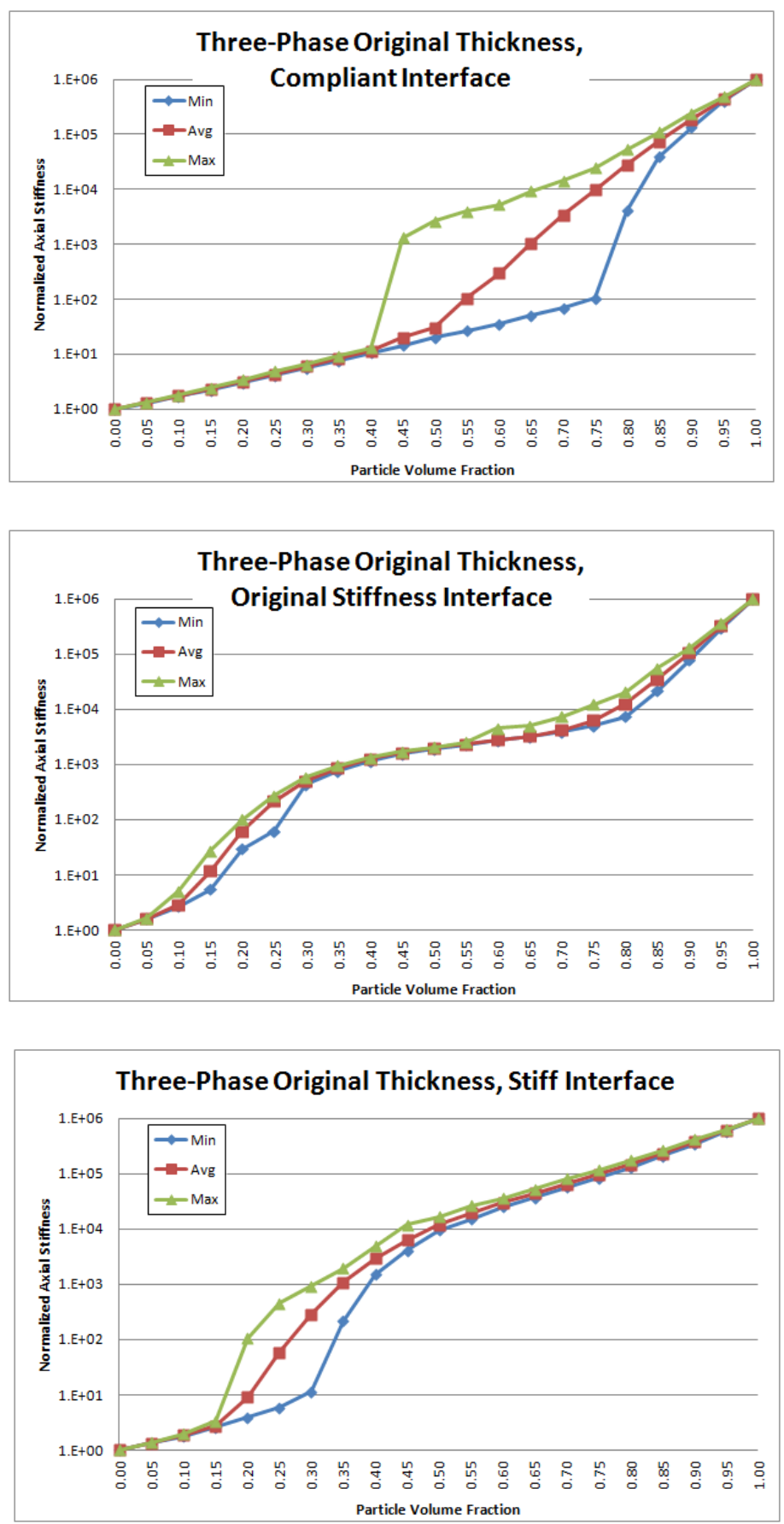

Figure 6.5: Simulated effective elastic axial stiffness (normalized minimum, average, and maximum values) for top: [Original Thickness, Compliant]; middle: [Original Thickness, Original Stiffness]; and bottom: [Original Thickness, Stiff] 

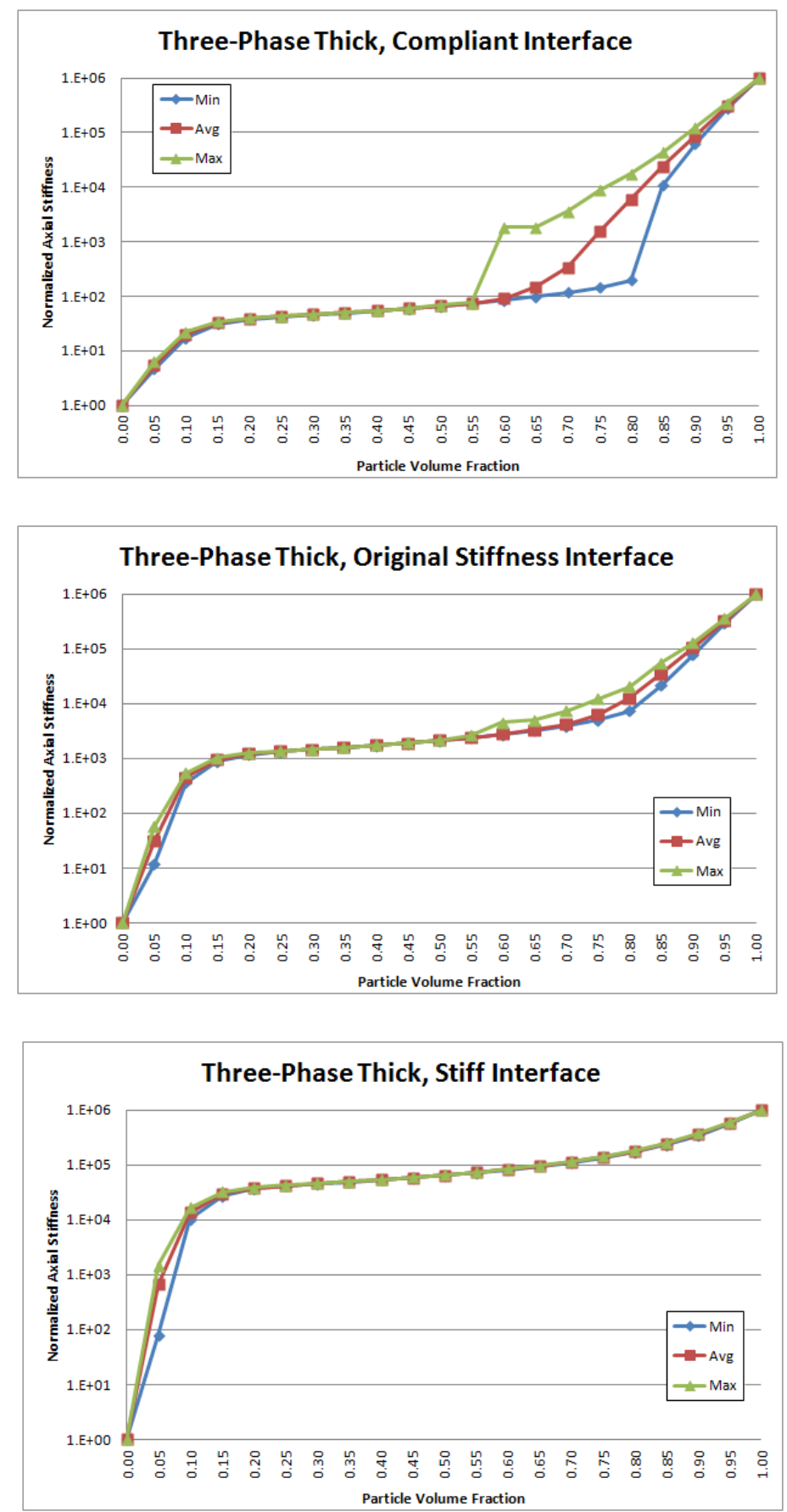

Figure 6.6: Simulated effective elastic axial stiffness (normalized minimum, average, and maximum values) for top: [Thick, Compliant]; middle: [Thick, Original Stiffness]; and bottom: [Thick, Stiff] 


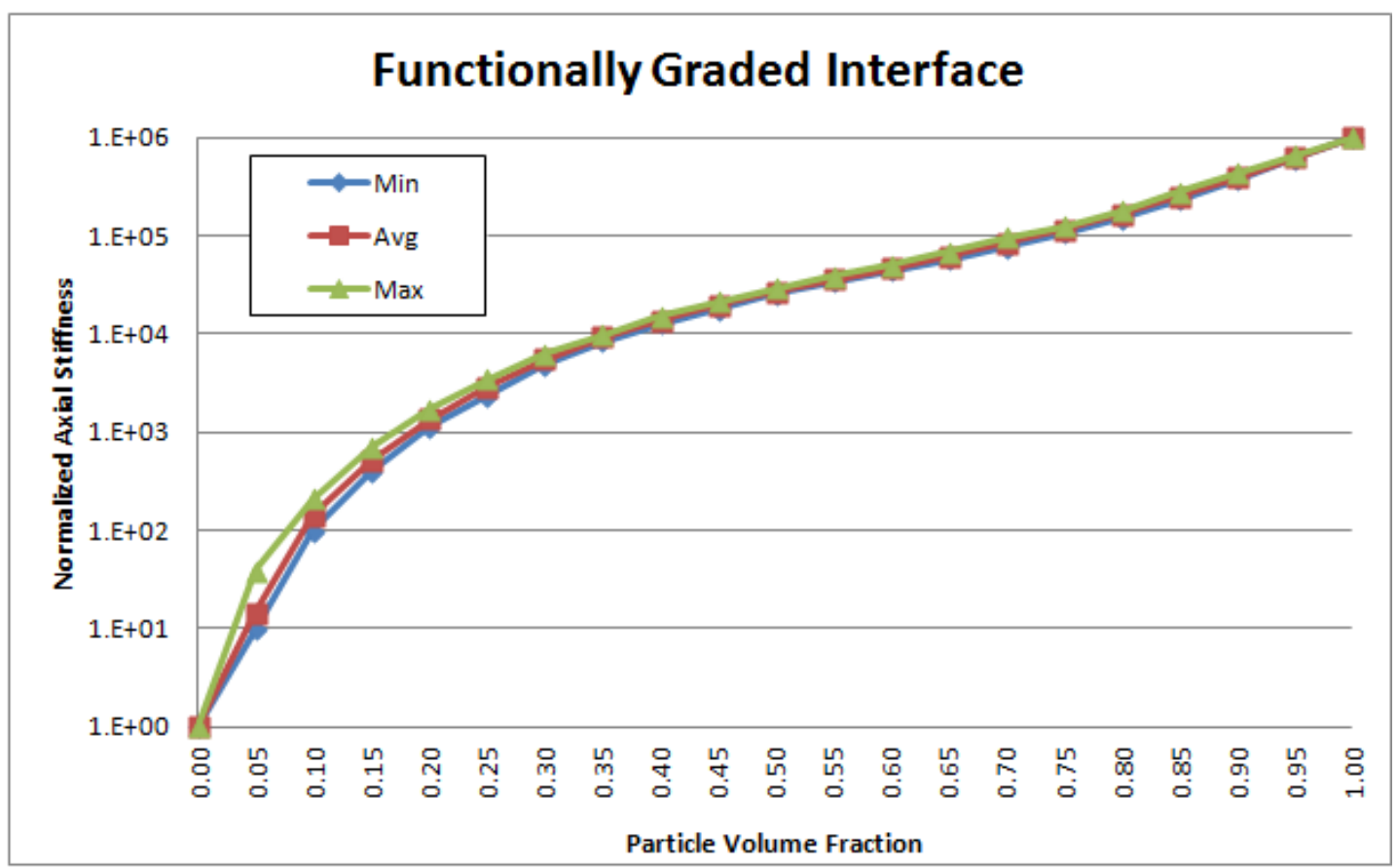

Figure 6.7: Simulated effective elastic axial stiffness (normalized minimum, average, and maximum) for five-phase composite with a gradient interface.

\subsection{Functionally Graded Interface}

To further investigate the effect of the interface on mechanical behaviors in nanocomposites, a functionally graded (FG) interface was created using three interface layers. A $2 \times 2 \times 2$ particle was first surrounded by one layer of a stiff interface material with a thickness equal to half the particle diameter. The particle and first interface layer were then surrounded by another layer of the original stiffness interface, again half the particle diameter thick. Finally, the particle and two layer interface were surrounded by a third layer of compliant interface with the same thickness as the other layers. The stiffness values for each layer of interface are the same as presented earlier in this chapter. The minimum, average, and maximum axial elastic stiffness values for 300 microstructural realizations at each volume fraction from 0 to 1 in 0.05 steps are shown in fig. 6.7.

To observe the effect of a functionally graded interface, fig. 6.8 compares the 


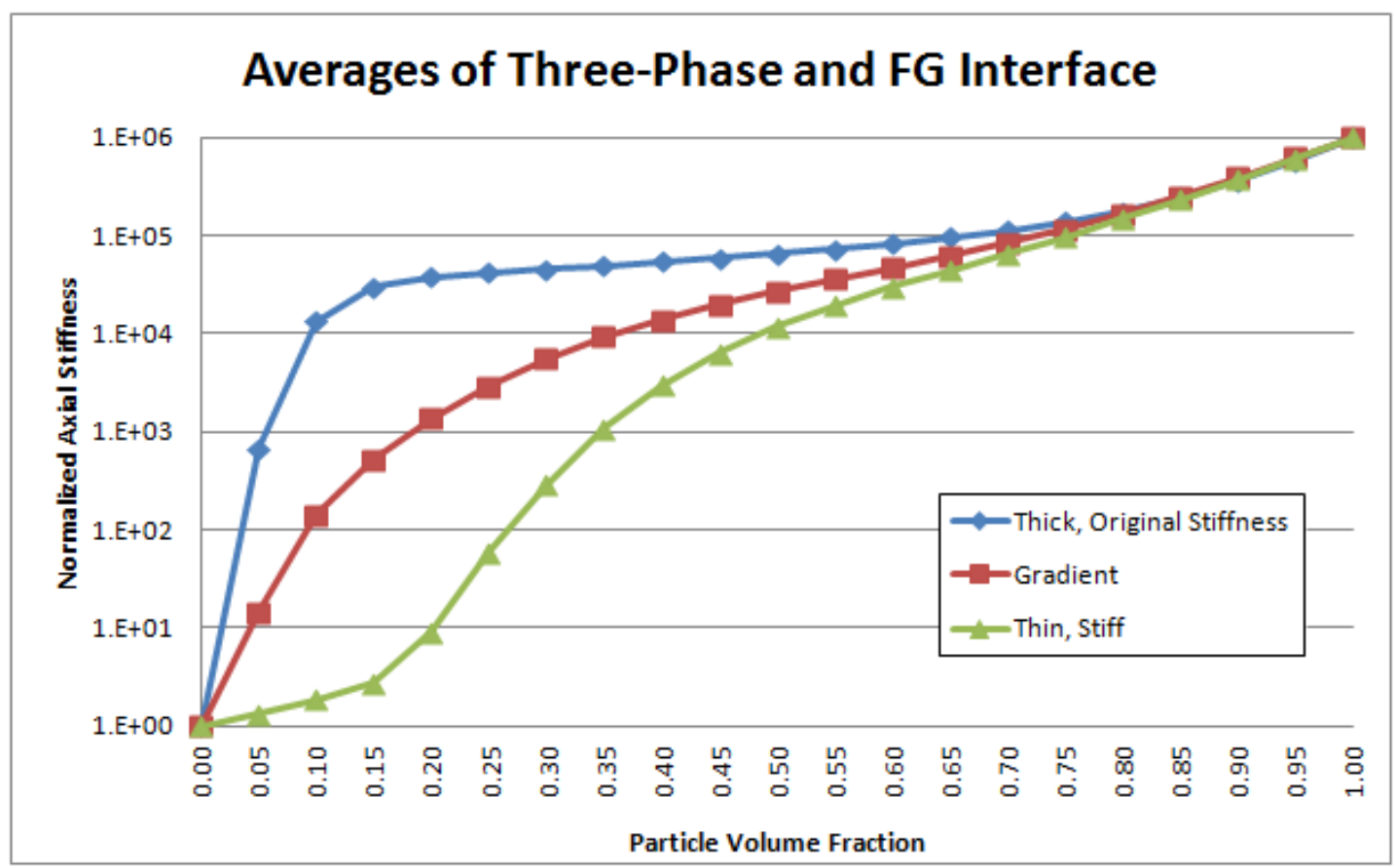

Figure 6.8: Simulated effective elastic axial stiffness for five-phase composite with a gradient interface; three-phase composite with a thick, original stiffness interface; and three-phase compsite with a thin, stiff interface.

average from the FG interface to the average curve of the [thick, original stiffness] interface composite and the [thin, stiff interface] composite from the previous sections.

\subsection{Summary and Conclusions}

Distinct to the compliant interface is the lack of low volume fraction percolation effect and the delayed effect of the apparent percolation at the high volume fraction with increasing thickness. Figure 6.9 top shows the average effective elastic axial stiffnesses of the three variations in the compliant group. As the compliant interface region increases in thickness, the first possible volume fraction where random microstructural effects contribute, also increases, $0.55,0.60$, and 0.65 , respectively.

With a stiff interface, the apparent percolation effect is only visible in the low volume fraction regime. Figure 6.9 bottom shows the average effective elastic axial stiffnesses of the three variations in the stiff group. In contrast to the compliant inter- 

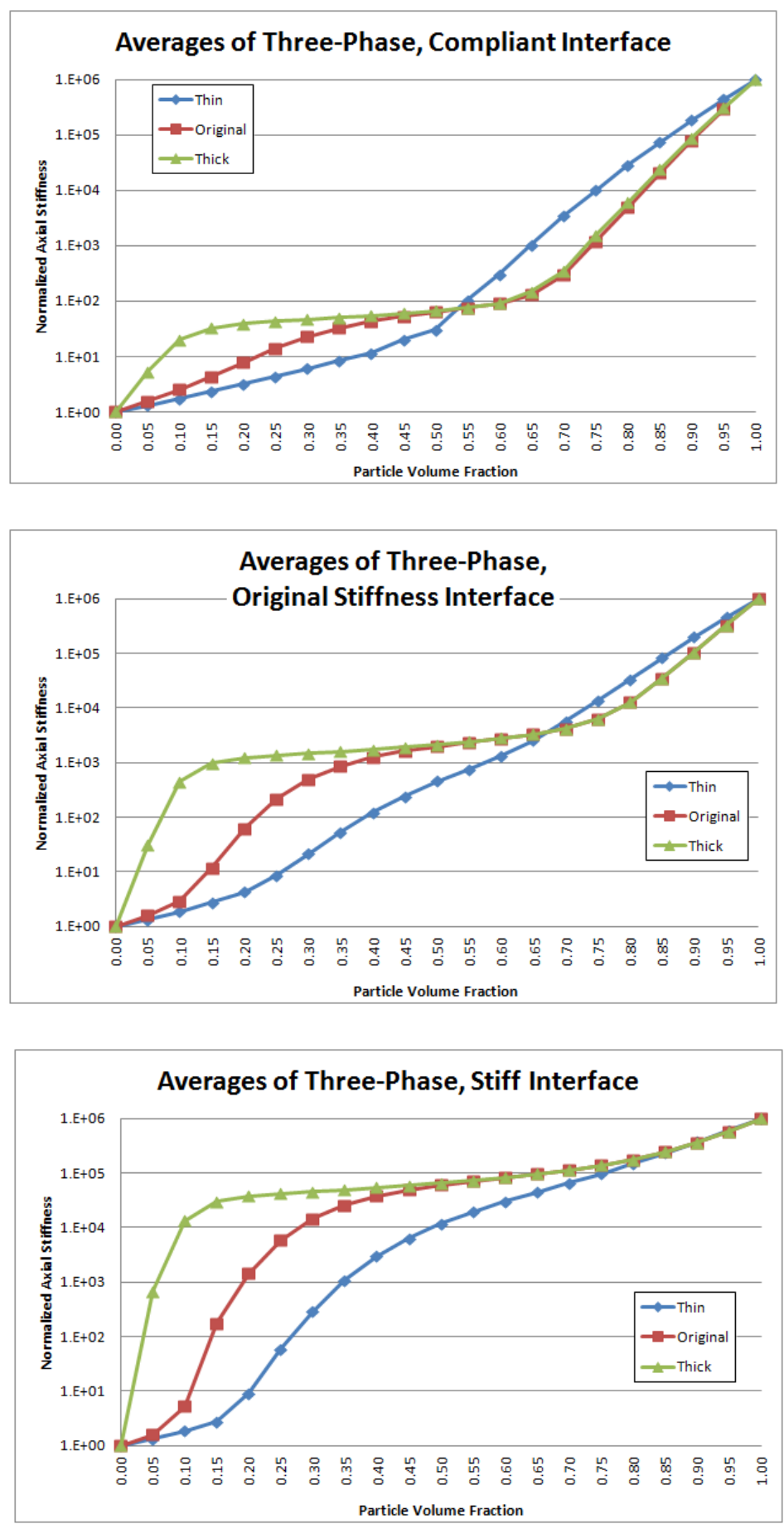

Figure 6.9: Simulated average effective elastic axial stiffnesses for three-phase composite in top: compliant group; middle: original stiffness group; and top: stiff group. 
face, as the interface region increases in thickness, the apparent percolation threshold decreases, 0.20, 0.10, and 0.05, for thin, original, and thick, respectively.

When the interface stiffness lies between these two extremes, original stiffness, no high volume fraction percolation behavior is observed for a thick or a thin interface, but apparent percolation thresholds in the low volume fraction regime decrease with increasing thickness; $0.20,0.10$, and 0.05 ; for thin, original, and thick, respectively. Figure 6.9 middle shows the average effective elastic axial stiffnesses of the three variations in the original stiffness group.

When the interface region is 'too' compliant, the formation of particle-interface stacks does not constitute a connected microstructure that is significantly different from particle widely dispersed in the matrix. However, as this compliant interface is made thicker it contributes a more significant volume fraction of a third phase and increases the overall composite stiffness at earlier volume fractions.

Figure 6.10 shows the average effective elastic axial stiffnesses of the variations in thickness with changing stiffness. The early appearance of the apparent percolation threshold at the high volume fractions with a thin interface may be a result of the persistence of the matrix material to a relatively high volume fraction, so that what appears to be the high volume fraction percolation (particle stacks) may be delayed formation of particle-interface stacks within the matrix materia, as shown in fig. 6.10 topl. The thick interface shows an apparent percolation threshold that is the same as the composite with an original stiffness interface, see fig. 6.10 bottom; the matrix material is completely gone by this point and the effect is due to particle stacks. The mechanisms behind the original thickness, compliant interface is more difficult to assess. It may be that because the interface stiffness is relatively close to that of the matrix that the effect is again that this represents a delay in the formation of composite particle stacks, rather than particle stacks.

When the interface region is 'too' stiff, the particle-interface stacks that form at 

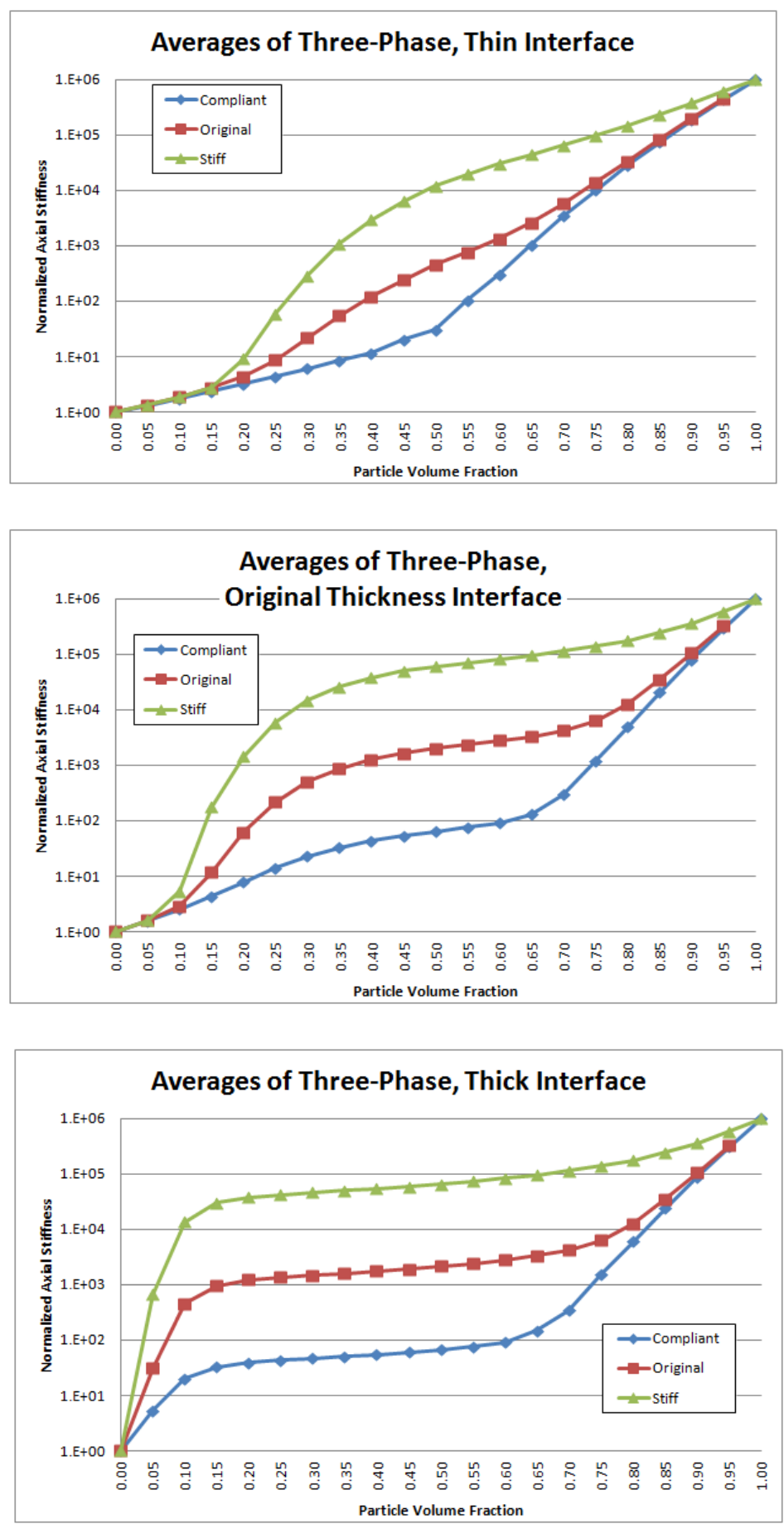

Figure 6.10: Simulated average effective elastic axial stiffnesses for three-phase composite in top: thin variation; middle: original thickness variation; and top: thick variation. 
the lower volume fractions produce a significantly higher jump in stiffness than a less stiff interface which lessens the effect of the particle stacks that form at the higher volume fractions. In this case, the effect of increasing the thickness is to facilitate the formation of particle-interface stacks earlier.

Interface effects are of most interest with respect to the low volume fraction regime, where these effects have been experimentally observed. In this region, interface thickness is almost purely geometry. More interface per particle implies overall stiffness is increased at lower particle volume fractions and connected particle-interface stacks occur at lower volume fractions. Increasing interface stiffness has more of a composite mechanics effect, i.e., the contribution of a stiffer third phase and little effect on the apparent percolation threshold. 


\section{Chapter 7}

\section{Two- And Three-Phase Continuum Model}

The geometry of the model used to generate the previous results closely parallels classic site-lattice work in percolation. Within the random checkerboard model, particles were assigned spatial positions similar to grid points on a cubic lattice. A major drawback to this implementation is its inability to model the microstructural response of stacked or aligned particles with any degree of overlap. Figure 7.1 illustrates a cubic particle with a side length of two spatial locations can have an overlap spacing of one spacial location in all directions. Extending the model to include this overlap is a step beyond the discrete lattice grid models, moving toward a percolation model with more of a continuum, off lattice site, perspective.

To allow for this possible overlap of particle stacks and alignment, the simulation code that placed random particles was modified. As a result of this style of placement, with a uniform particle size and no shifting of particle placement, it is not possible to simulate volume fractions over the full range. For the $2 \times 2 \times 2$ particle in a

$48 \times 48 \times 48 \mathrm{RUC}$, the simulation code could only generate volume fractions up to 0.65. As an initial comparison, the overlap results, referred to here as the Continuum Model results, are compared to the original lattice based model, referred to as the Lattice Model. 


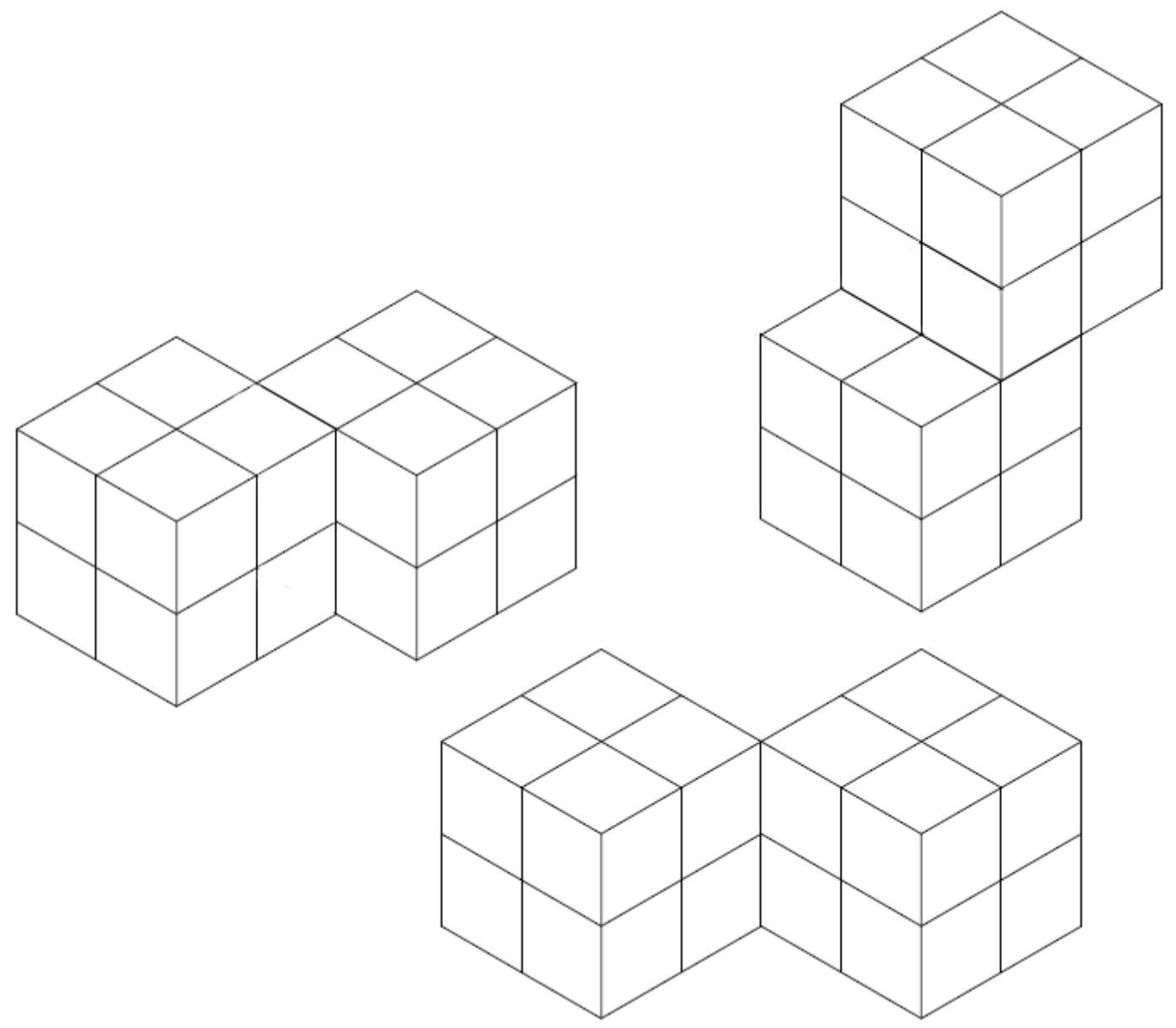

Figure 7.1: Examples of possible overlap scenarios within the RUC

\subsection{Continuum versus Lattice}

\section{Two-Phase Composite}

Random microstructures for the two-phase (matrix and particle) composite were simulated for volume fractions from 0 to 0.65 in steps of 0.05 ; 300 microstructural realizations were generated for each volume fraction using the continuum model. GMC was used to approximate the effective elastic properties associated with each microstructure. Figure 7.2 compares the minimum, average, and maximum values of the effective elastic axial stiffness, $E_{11} / E_{\text {matrix }}$, for the continuum and lattice models.

The continuum model appears to show a delay in the apparent percolation thresh- 


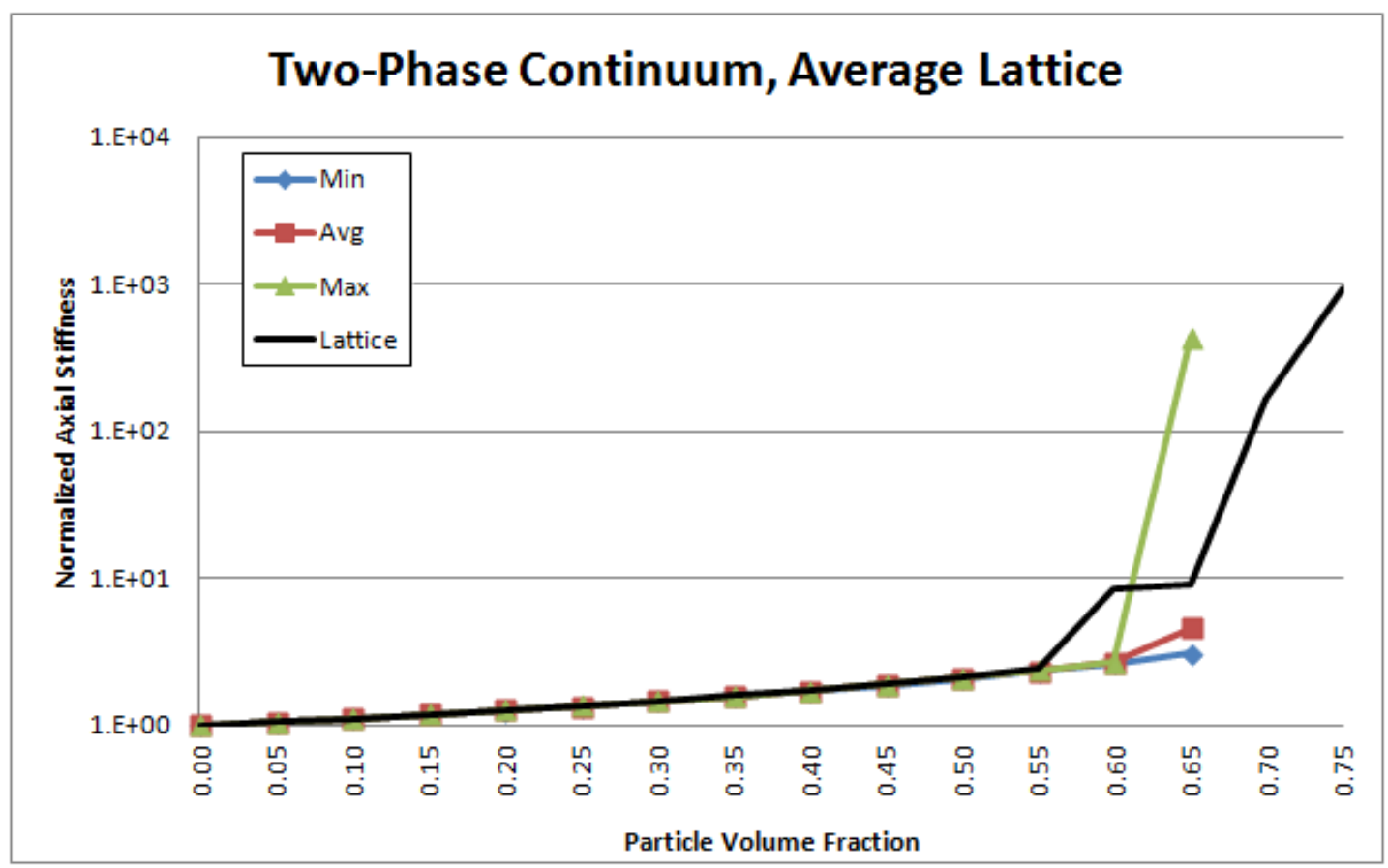

Figure 7.2: Two-phase $2 \times 2 \times 2$ particle composite: Continuum and Lattice model comparison.

old and lower maximum moduli for the values beyond percolation. This is consistent with our understanding that mechanically, in GMC, a continuous stack where particle centers are not all aligned, i.e., partial overlapping, provides less reinforcement than the fully aligned stacks.

\section{Three-Phase Composite}

Random microstructures for the three-phase (matrix, particle, and interface) composite were simulated at volume fractions from 0 to 0.65 in steps of $0.05 ; 300$ microstructural realizations were generated for each volume fraction using the continuum model. GMC was used to approximate the effective elastic properties associated with each microstructure. Figure 7.3 compares the three-phase minimum, average, and maximum effective elastic axial stiffnesses of the continuum model and the lattice model. There are no significant differences between the two. This is likely because the particle volume fractions are so low that percolation effects are due to interface-particle rather 


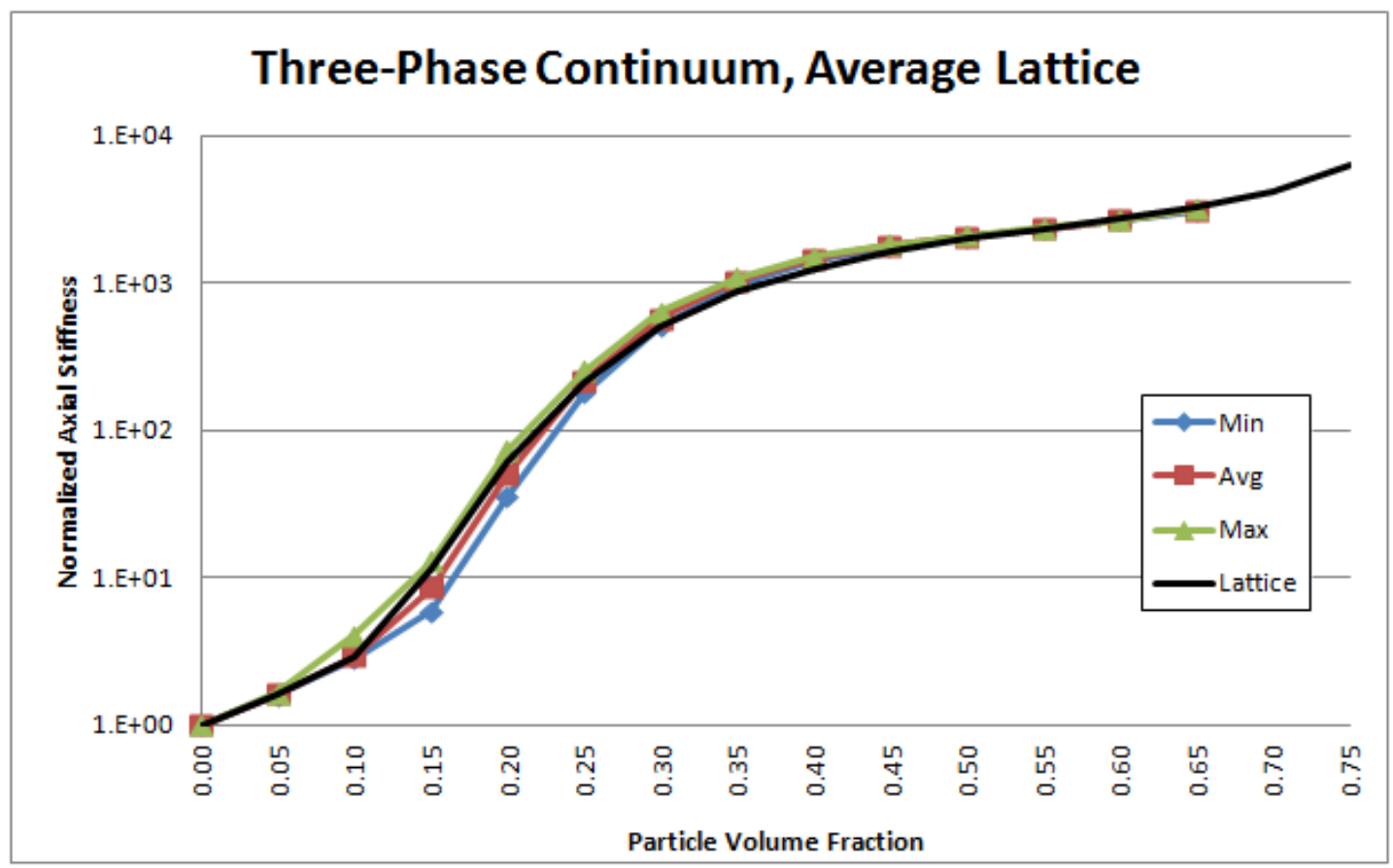

Figure 7.3: Three-phase $2 \times 2 \times 2$ particle composite: Continuum and Lattice model comparison.

than particle-particle connections and so the probability of particles even partially overlapping is low.

In a subsequent chapter, the Continuum Model is used to investigate higher aspect ratio particles. The overlap construction allows the longer rods to line up overlapping longitudinally as well as transversely adjacent, but off-set, to one another. 


\section{Chapter 8}

\section{High Aspect Ratio Particles}

The modeling approach taken in this work offers a significant advantage over classic percolation models in that it can be used to model micromechanical effects beyond the formation of a connected particle microstructure. One important micromechanical effect is the influence of the aspect ratio of the included phase. Classic micromechanics predicts that the stiffness of a composite containing aligned high aspect ratio particles, aligned in the long direction, is greatest in the direction of the alignment [46].

\subsection{Two-Phase Composite with Varying Aspect Ratios}

Random microstructures for a two-phase composite containing rods with aspect ratios of $6: 1,8: 1$, and $12: 1$, respectively, were simulated in steps of $0.05 ; 300$ microstructural realizations were generated for each volume fraction using the continuum model. Particle subcell dimensions made overlap possible along both longitudinal and transverse axes. The long axis of these rods runs parallel to the 1-direction. GMC was used to approximate the effective elastic properties associated with each microstructure. Figures 8.1 - 8.3 plot the normalized minimum, average, and maximum values of the longitudinal elastic stiffness, $E_{11} / E_{\text {matrix }}$, versus volume fraction for each aspect ratio.

For the two-phase composite, the formation of continuous particle stacks results in change in composite stiffnesses of several orders of magnitude. As a result, it is possible to calculate the percentage of the 300 simulations that exhibit this jump and relate these values to the percolation threshold. In other words, define the percolation threshold as the volume fraction, beyond which, all of the microstructures display this 
connectivity. Figure 8.4 plots the probability of finding a percolated, continuous stack microstructure in the population of simulated microstructures. In each of the cases above, it was possible for the higher aspect ratio particles to fit "evenly" in the RUC. In other words, 6, 8, and 12 all divide 48 with no remainder. As a result, percolated microstructures, in the form of continuous stacks, could form. Figure 8.4 highlights the expected trend that percolation thresholds decrease with increasing aspect ratio.

Random microstructures for two-phase composite containing rods with aspect ratio of $5: 1,7: 1$, and 10:1 were also simulated in steps of $0.05 ; 300$ microstructural realizations were generated for each volume fraction using the continuum model. Particle subcell dimensions again allowed for possible overlap along both longitudinal and transverse axes. The long axis of these rods runs parallel to the 1-direction. GMC was used to approximate the effective elastic properties associated with each

microstructure. Figure 8.5 plot the normalized minimum, average, and maximum values of the longitudinal elastic stiffness, $E_{11} / E_{\text {matrix }}$, at each volume fraction for top: 5:1, middle: 7:1, and bottom: 10:1 aspect ratios.

For these aspect ratios, the rod dimensions do not divide the RUC evenly and so, given the assumptions and limitations of the model, a percolated microstructure in the form of a continuous stack, is not possible. Overlap and the ability to stack particles into stacks that almost span the RUC resulted in higher composite stiffnesses with increasing aspect ratio. This effect is what would be expected from classic mean-field micromechanics theory.

\subsection{Three-Phase Composite, Influence of Aspect Ratio}

Random microstructures for a three-phase (matrix, particle, and interface) composite with aspect ratio of 5:1, were simulated in steps of $0.05 ; 300$ microstructural realizations were generated for each volume fraction using the continuum model. GMC was used to approximate the effective elastic properties associated with each microstruc- 


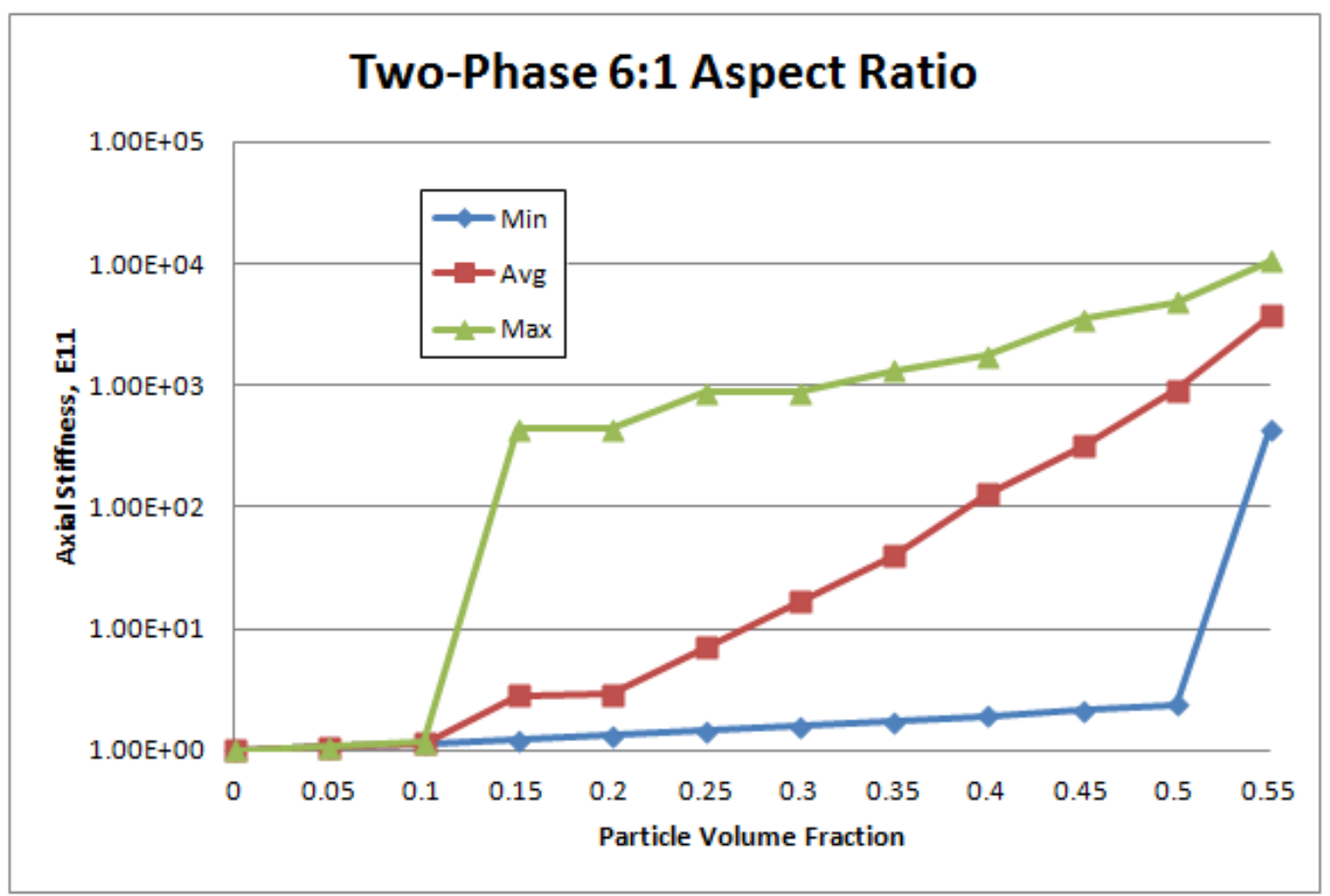

Figure 8.1: Simulated effective elastic axial stiffness for two-phase composite with 6:1 aspect ratio particles, longitudinal and transverse overlap.

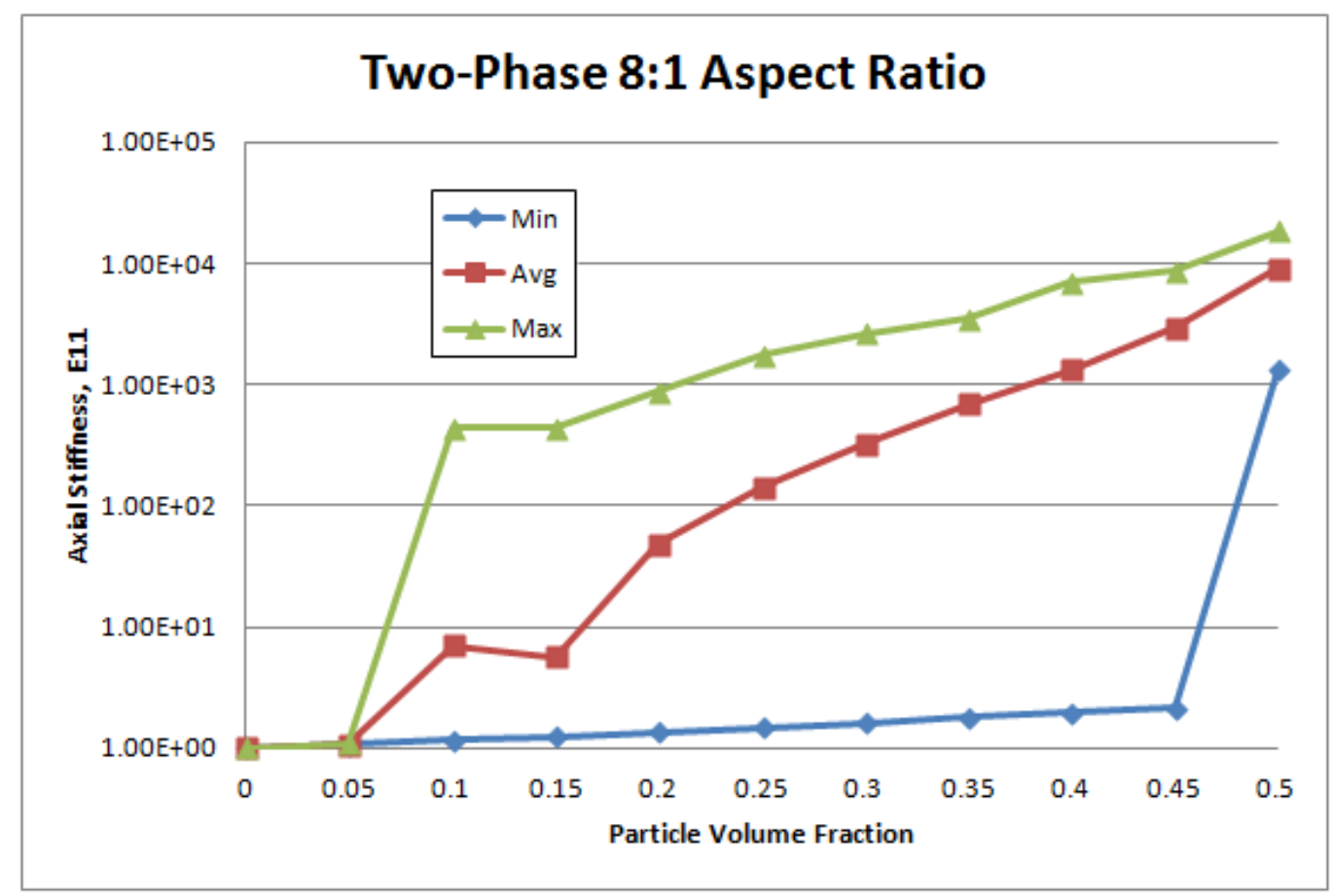

Figure 8.2: Simulated effective elastic axial stiffness for two-phase composite with 8:1 aspect ratio particles, longitudinal and transverse overlap. 


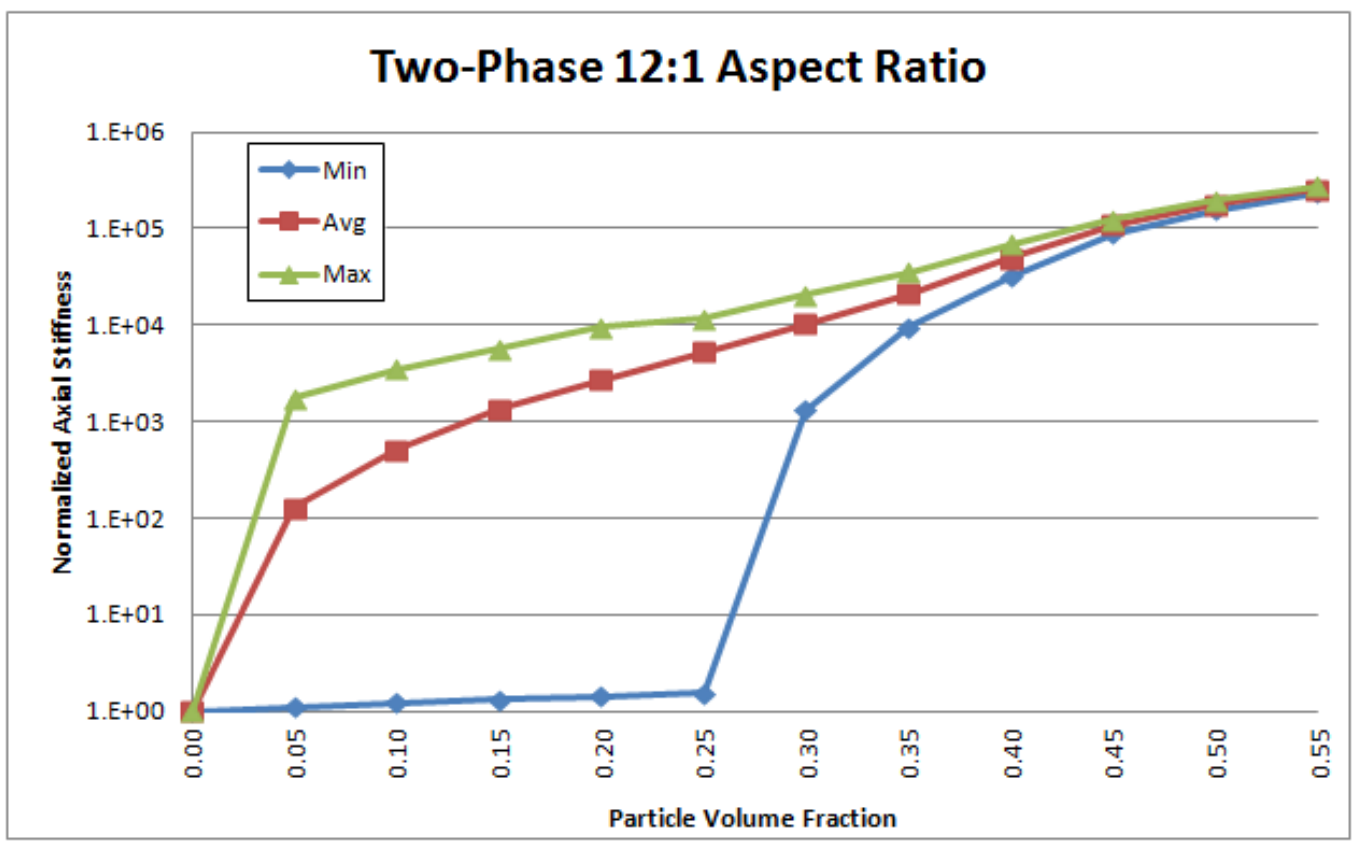

Figure 8.3: Simulated effective elastic axial stiffness for two-phase composite with 12:1 aspect ratio particles, longitudinal and transverse overlap.

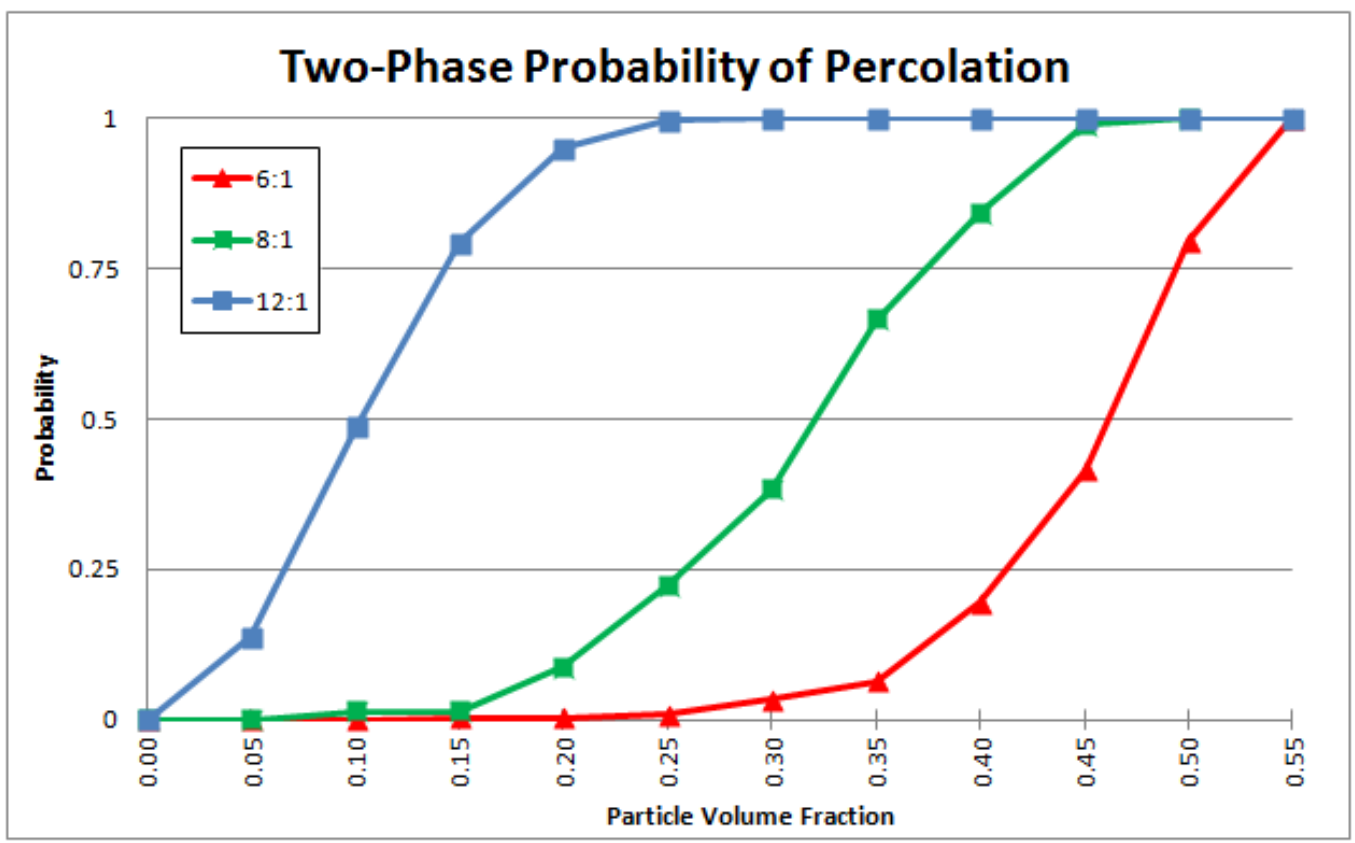

Figure 8.4: Probability of percolated microstructures for two-phase composite with particles of various aspect ratio. 

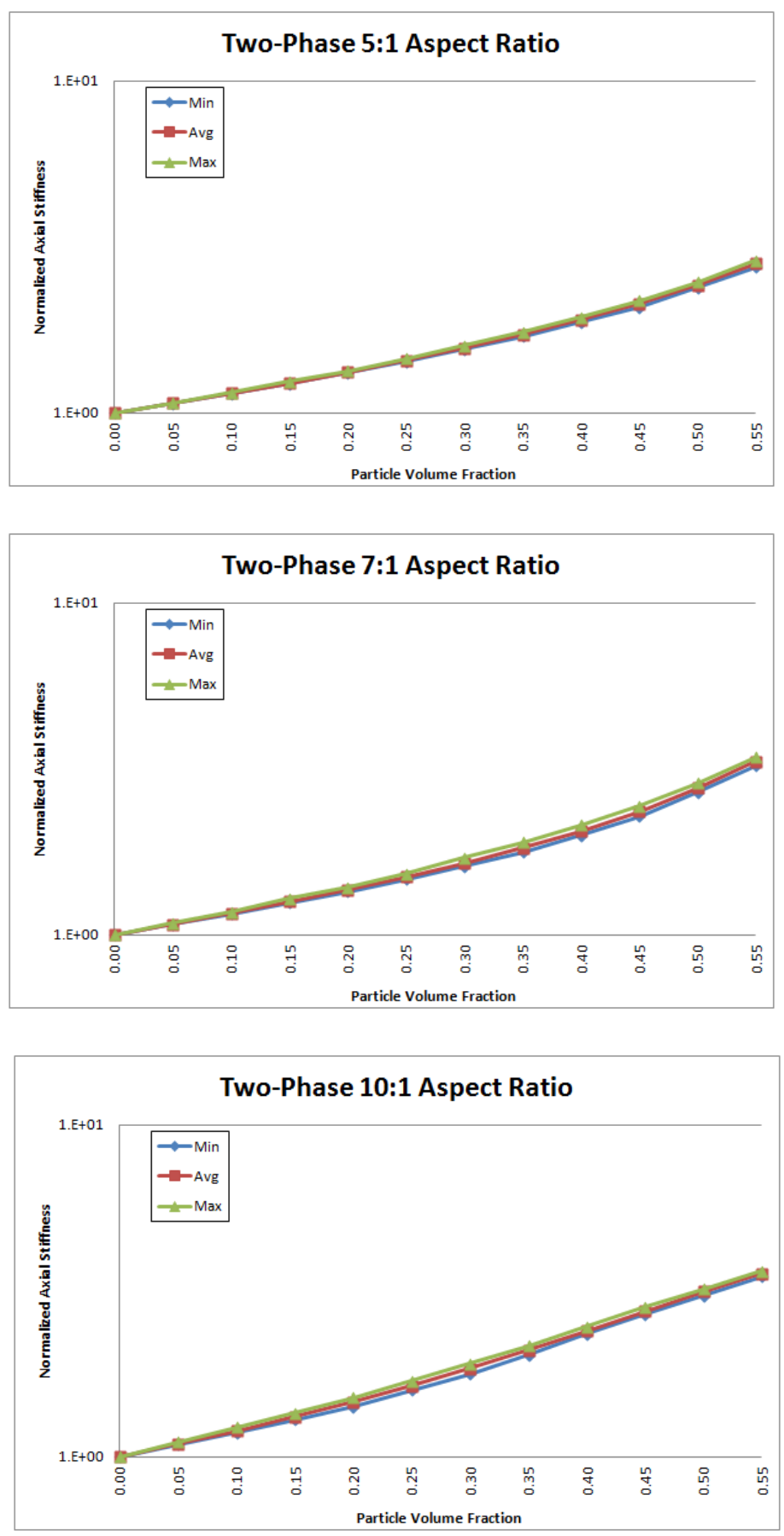

Figure 8.5: Simulated effective elastic axial stiffness for two-phase composite containing particles with top: 5:1 aspect ratio; middle: 7:1 aspect ratio; and bottom: 10:1 aspect ratio. 


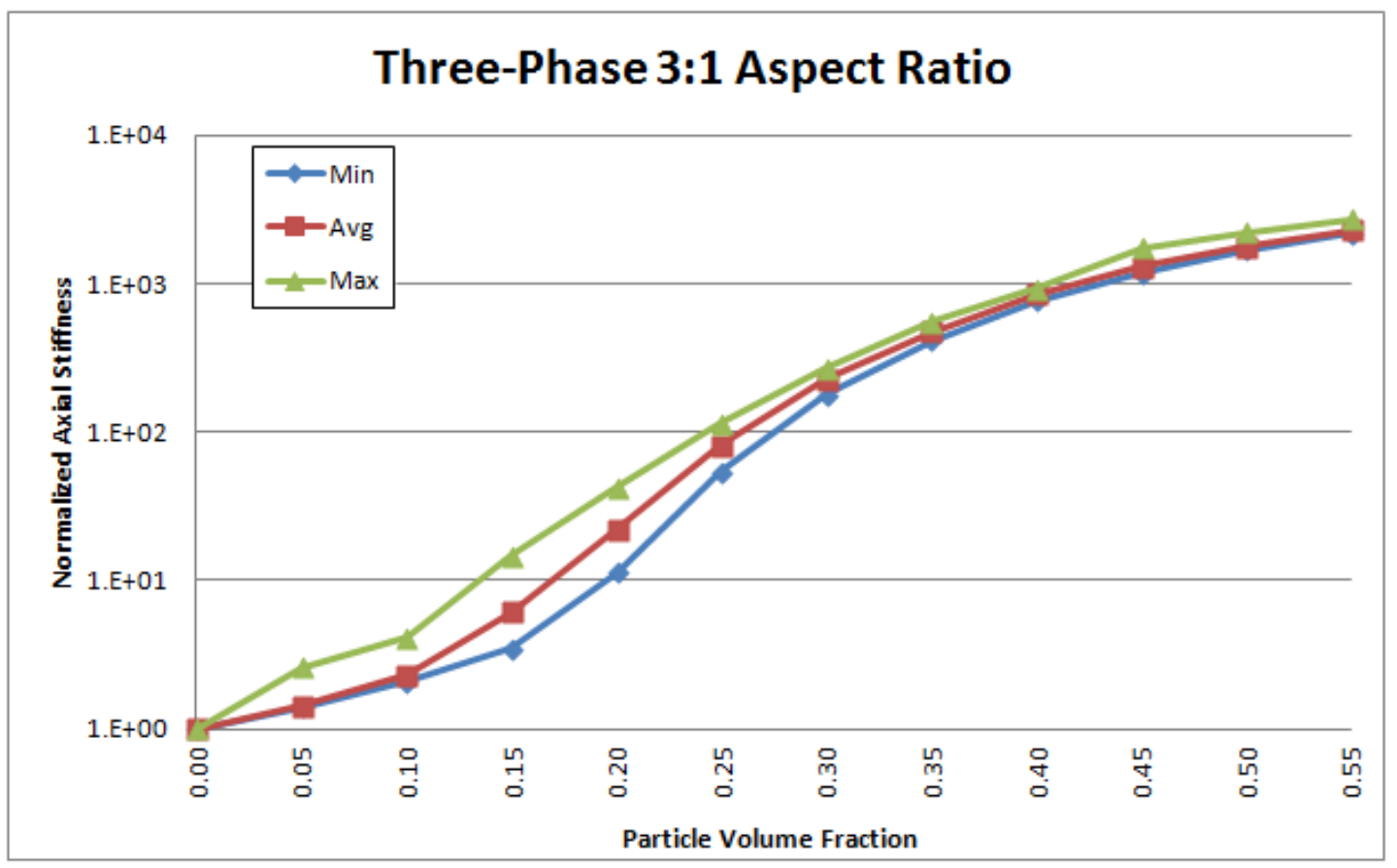

Figure 8.6: Simulated effective elastic axial stiffness for three-phase composite with 3:1 aspect ratio particles, longitudinal and transverse overlap.

ture. Figure 8.7 plots the minimum, average, and maximum values of the normalized effective elastic axial stiffnesses, $E_{11} / E_{\text {matrix }}, E_{22} / E_{\text {matrix }}$, and $E_{33} / E_{\text {matrix }}$ at each volume fraction. Figure 8.8 compares the three axial moduli for the 5:1 aspect ratio particles. As expected, the average composite stiffness is greatest in the direction of alignment.

Further increasing the aspect ratio to 8:1 results in curves with a similar shape but slightly lower apparent percolation threshold to the composite with 5:1 aspect ratio particles. Minimum, average, and maximum normalized curves are shown in fig. 8.9. With the longer particles, but similarly thick interface regions, this reflects the greater ease of forming composite particle-interface stacks with longer particles. The two average curves for the three-phase 8:1 and 5:1 aspect ratio particles are compared in fig. 8.10. The results show an increase in overall stiffness with the higher aspect ratio.

The effect of higher aspect ratio aligned particles is consistent with the underlying 


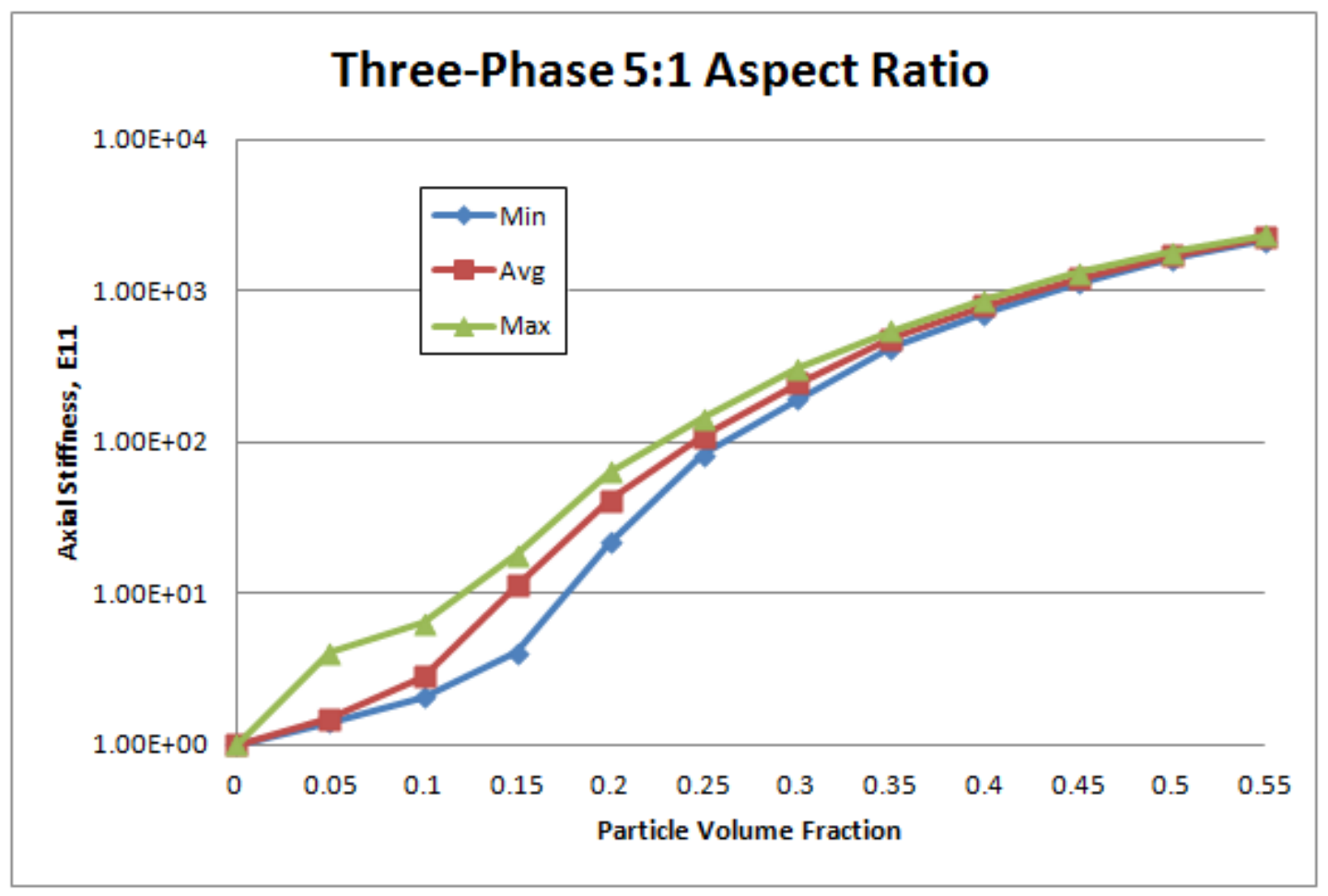

Figure 8.7: Simulated effective elastic axial stiffness for three-phase composite with 5:1 aspect ratio particles, longitudinal and transverse overlap.

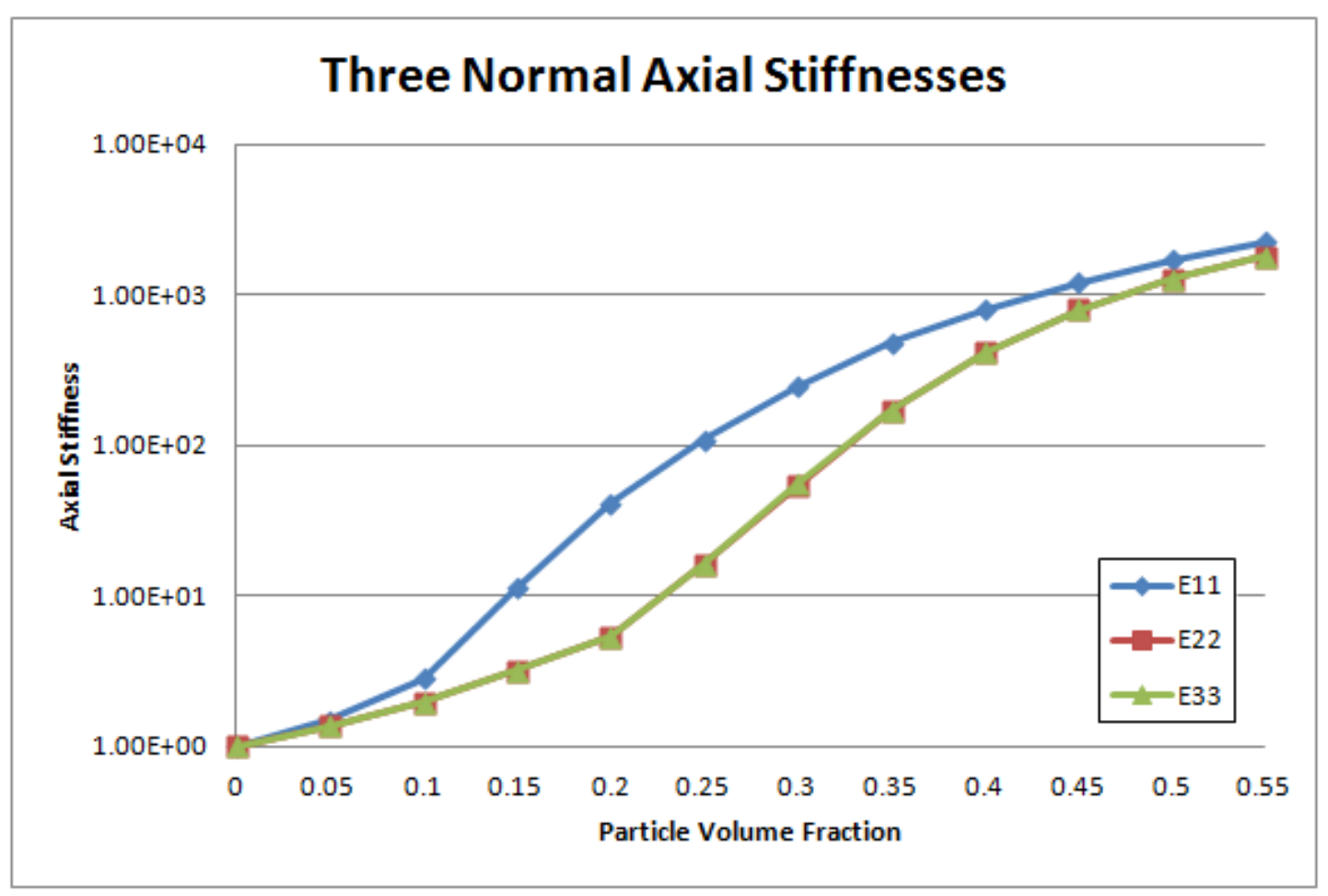

Figure 8.8: Simulated effective elastic axial stiffness averages for three-phase composite with 5:1 aspect ratio particles, longitudinal and transverse overlap. 


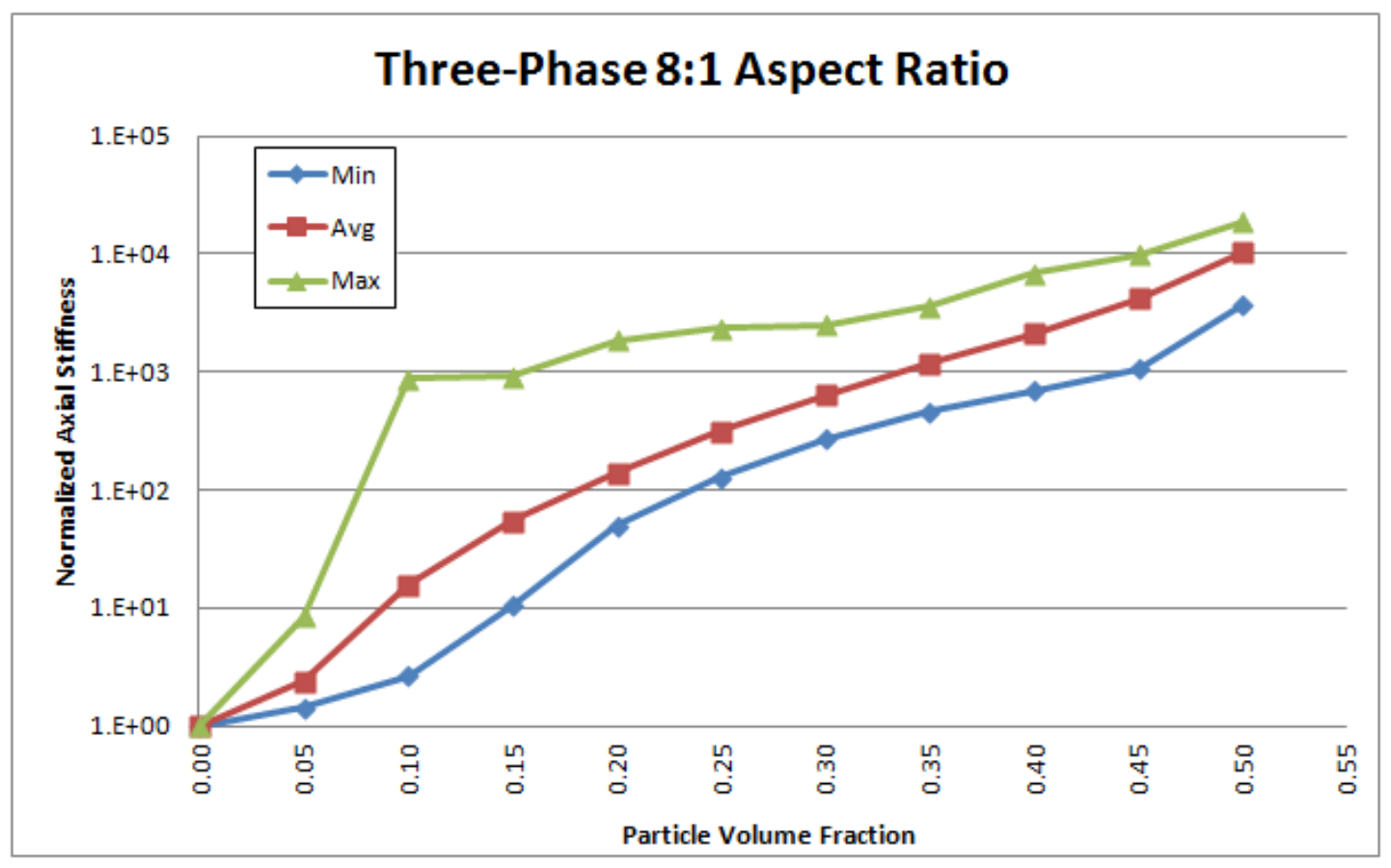

Figure 8.9: Simulated effective elastic axial stiffness for three-phase composite with 8:1 aspect ratio particles, longitudinal and transverse overlap.

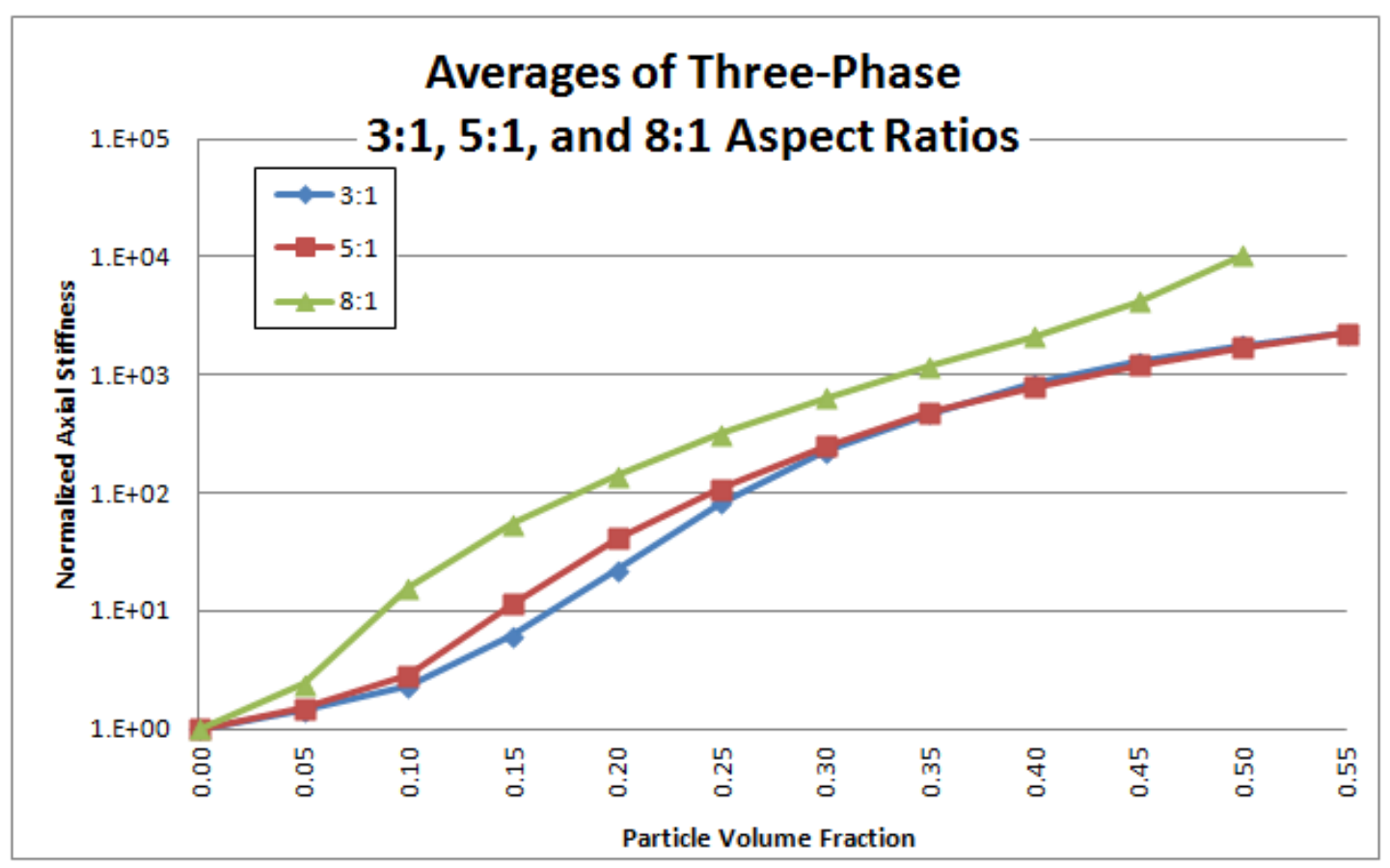

Figure 8.10: Simulated effective elastic axial stiffness averages for three-phase composite with $3: 1,5: 1$, and 8:1 aspect ratio particles, longitudinal and transverse overlap. 
micromechanics theory that predicts greater axial composite stiffness in the long direction of the included phase. The effect of aspect ratio on the apparent percolation threshold in the low volume fraction regime is an earlier percolation threshold in addition to the increased composite stiffness. 


\section{Chapter 9}

\section{Summary AND Discussion}

Experimental work shows evidence of percolation-like effects for mechanical properties in polymer nanocomposites. Models of percolation phenomena, often power-laws, have been extensively developed for electrical conductivity in composite materials and, as a result, are commonly applied to these mechanical effects as well. A weakness of these models is that they contain very little mechanics other than an estimate of the volume fraction where percolated, or connected, microstructures develop. These estimates, in the form of a percolation threshold parameter in the models, are either established independent of the material systems or used as an empirical parameter for a curve fit. This limits the value of the model as a predictive tool that can take micromechanical effects into consideration. The use of classic mean-field micromechanics models to capture the mechanics of these systems, however, does not offer a method to describe percolation or the mechanical effects of randomness in the composite microstructure.

In this work, rather than attempting to estimate critical volume fractions a priori to use in predicting mechanical properties, the modeling paradigm was reversed. Simulated effective elastic composite properties were used to track the effects of the evolution of a mechanically percolating microstructure and indicate the presence of percolation effects. This was done in a Monte Carlo framework, where the effective properties were calculated using a computational micromechanics model for multiple random microstructural realizations. The Generalized Method of Cells micromechanics model was used to approximate the effective elastic properties. Simulations were 
performed for both a macro-scale (two-phase) and nano-scale (three-phase) discontinuously reinforced composite consisting of cubic metallic particle embedded in a polymeric matrix.

Apparent percolation effects were clearly visible in the simulation of the twophase composite. A percolation threshold could be identified as the volume fraction, of approximately 0.80 , beyond which all of the simulated microstructures displayed percolation microstructures. Connected microstructures took the form of continuous particle stacks, which in the context of the repeating unit cell model, produced the effect of a continuous fiber composite. The two-phase material did not, however, capture the low volume fraction percolation effects that have been observed for nanocomposite materials.

When the simulations were modified to include a third phase, an interface region which reflects one of the effects of scale in nanocomposites, the simulations showed evidence of a second region of percolation-like effects at a significantly lower percolation threshold. These apparent percolation effects appear due to the formation of microstructural stacks made of both particles and interface material that assemble within the compliant matrix. This has been referred to as a pseudo-percolation effect, connections via particles and interface, but still results in significant mechanical reinforcement to the composite material. Because the effects of pseudo-percolation occur as the volume fractions of the three phases are also changing: increasing particle and interface, and decreasing matrix volume fraction, its role as a dominant effect is limited to a small range and small values of volume fraction. However, these results do suggest a mechanism that might contribute to the experimentally observed significant enhancement of properties at low volume fractions in nanocomposites.

In addition to the significant result of designing a modeling framework that can duplicate the observed experimental effects using probabilistic and micromechanical analysis, the advantage of this approach is that other micromechanical and mi- 
crostructural effects can be included, in a consistent manner, in the model.

The effect of interface stiffness and thickness was investigated for the three-phase composite. By adjusting the thickness of the interface, the apparent low volume fraction percolation threshold could be adjusted; thicker interfacial regions lowered the threshold values. However, in addition to a lower percolation threshold, thicker interface regions affect the fundamental of the composite, replacing the matrix material with a stiffer third phase at a lower volume fraction and increasing the overall stiffness.

When the stiffness of the interface was varied, the apparent percolation threshold was not affected; however, the overall effective stiffness of the composite was changed depending on the interface stiffness. With a sufficiently stiff interface, the composite stiffness reached very high levels at relatively low volume fractions and so the secondary percolation effects, due to the formation of particle stacks, had very little additional influence on the properties. For a sufficiently compliant interface, the particle-interface stacks provided little additional reinforcement.

A second microstructural mechanism that can be studied using this modeling framework is the influence of aspect ratio of the included phase. In both the two- and three-phase composite models apparent (three-phase) and measurable (two-phase) percolation thresholds were shifted to lower volume fractions with higher aspect ratios of the included particles. This is consistent with micromechanical theory and experiments on aligned high aspect ratio fibers; both the increase in stiffness and the lower threshold values.

These results highlight the usefulness of this modeling framework and approach as a predictive tool to study these materials. As a micromechanics approach, it is novel in its inclusion of scale effects, i.e. scaling the size of the interface to the size of the particle. Classic micromechanics models are, in general, scale invariant because they depend on volume fraction, a dimensionless parameter. As a probabilistic approach 
or an extension to percolation theory, this approach includes adjustable physical and material parameters that can be realistically used to match experimental data, suggests mechanisms that might be linked to specific effects, and could help suggest additional experimental work. 


\section{Chapter 10 FUTURE WORK}

In this research, a modeling framework was designed that combined a micromechanics model with a Monte Carlo simulation approach to describe the evolution of mechanical percolation effects in nanocomposites with random microstructures. This model used a three-dimensional repeating unit cell (RUC) model to calculate effective elastic properties. By applying periodic boundary conditions to these RUCs, an infinite material response is simulated. In this first stage of the work, a nanocomposite was modeled by including an interface region, which is thought to contribute to the enhanced properties in nanocomposites. Scale was included in the model by linking interface thickness particle dimension. The significance of the modeling framework is that it can be used to include microstructural effects beyond that of the simple connectivity that characterizes percolation theory.

There are a number of clear extensions to, and applications of, the model that can be suggested.

- Future work for this research should include additional validation through comparison to experimental data. In particular, the cases where investigators, or theory, suggest additional details of the microstructure, e.g. interface geometry and properties.

- While the focus of this work was on allowing mechanical properties to predict the onset of percolation effects, it would be interesting to follow through with more specific geometric analysis of the microstructures. This could include more 
complex connectivity and two-point spatial correlation characterization.

- In this work, three-dimensional microstructures were simulated. Since percolation theory has been developed for both two- and three-dimensional materials, it would be useful to apply this approach to modeling two-dimensional materials. This will allow further connections of the model to existing techniques of simulation and theory for random materials. Simulating a two-dimensional material system will extend the model to continuous fiber composites.

- Similar to the anticipated two-dimensional analysis, adapting the model to simulate and capture finite size effects will also provide valuable feedback for nanocomposites. When the one dimension of the sample material approaches the size of the particle, the critical volume fraction is affected. This has been reported experimentally and a computational model capable of simulating such affects will provide more information with regards to particle size. This application will provide insight to the benefits, effects, and manufacture of thin films.

- The model developed in this research characterized particles with varying aspect ratios. Using the model to explore the effects of a distribution of aspect ratios might provide a more realistic material response. Additionally, while GMC is limited with respect to short fiber orientations, it would be interesting to model particles at $\pm 90^{\circ}$ orientations.

In conclusion, comparing experimental data to the predictions from the model proposed here, as well as exploring the mechanics based effects that are possible using this model, will help provide validation and verification of the consistency and usefulness of this model. 


\section{BIBLIOGRAPHY}

[1] Liu, H. and Brinson, L.C. "A Hybrid Numerical-Analytical Method for Modeling the Viscoelastic Properties of Polymer Nanocomposites" Journal of Applied Mechanics, 73, 758-768, 2006.

[2] Riu, H. and Brinson, L.C. "Simulation of interphase percolation and gradients in polymer nanocomposites" Composites Science and Technology, 69, 491-499, 2009 .

[3] Baxter, S.C. and Robinson, C.T. "Pseudo-percolation: Critical volume fractions and mechanical percolation in polymer nanocomposites" Composites Science and Technology, 71, 1273-1279, 2011.

[4] Celzard, A. McRae, E, Deleuze, C., Dufort, M., Furdin, G. and Marêché, J.F. "Critical concentration in percolating systems containing a high-aspect-ratiofiller" Physical Review B, 53(10), 6209-6214, 1996.

[5] Li, J. Kim, J-K. "Percolation threshold of conducting polymer composites containing 3D randomly distributed graphite nanoplatelets" Composites Science and Technology, 67, 2114-2110, 2007.

[6] Stauffer D. and Ahorony, A. An Introduction to Percolation Theory, Taylor \& Francis, London and Philadelphia, 1985.

[7] Balberg, I. and Binenbaum, N. "Computer study of the percolation threshold in two-dimensional anisotropic system of conducting sticks" Physical Review B, 28(7), 3799-3812, 1983.

[8] Baker, D.R., Raul, G, Sreenivasan, S., and Stanley, H.E. "Continuum percolation threshold for interpenetrating squares and cubes" Phys. Rev. E., 66, 046136-1: 046136-5, 2002.

[9] Consiglio, R., Baker, D.R., Paul, G. and Stanley, H.E. "Continuum percolation thresholds for mixtures of spheres of different sizes" Physica A, 319, 49-55, 2003. 
[10] Chiavazzo E. and Asinari, P. "Reconstruction and modeling of 3D percolation networks of carbon fillers in a polymer matrix" International Journal of Thermal Sciences, 49, 2272-2281, 2010.

[11] Oskouyi, A.B. and Mertiny, P. "Monte Carol model of the study of percolative thresholds in composites filled with circular conductive nano-disks" Engineering Procedia, 10, 403-408, 2011.

[12] Balberg, I., Binenbaum, N. and Wagner, N. "Percolation Thresholds in the Three-Dimensional Sticks System" 52(17), 1465-1468, 1984.

[13] Balberg, I. Anderson, C.H., Alexander, S. and Wagner, N. "Excluded volume and its relation to the onset of percolation" Physical Review B, 30(7), 39333943, 1984.

[14] Scher, H. and Zallen, R. "Critical Density in Percolation Processes" Journal of Chemical Physics, 53(9), 3759-3761, 1970.

[15] Torquato, S. Random Heterogenous Materials; Microstructure and Macroscopic Properties, Interdisciplinary Applied Mathematics, Eds. S.S. Antmann and L. Sirovich, Springer, N.Y. 2001.

[16] Golden, K. "Convexity and Exponent Inequalities for Conduction near Percolation" Phys. Rev. Lett. 65(24), 2923-2926, 1990.

[17] Celzard, A., and Marêché, J.F. "Non-universal conductivity critical exponents in anisotropic percolating media: a new interpretation" Physica A, 317, 305-312, 2003.

[18] Ouali, N. Cavaillé, J.Y., and Perez, J. "Elastic viscoelastic and plastic behavior of multiphase polymer blends" Plastics, Rubber and Composites Processing and Application, 16, 55-60, 1991.

[19] Favier, V., Chanzy, H. and Cavaillé, J-Y. "Polymer Nanocomposites reinforced by Cellulose Whiskers" Macromolecules, 28, 6365-6367, 1995.

[20] Dubief, D., Samain, E., Dufresne, A. "Polysaccharide Microcrystals Reinforced Amorphous Poly( $\beta$-hydroxyoctanoate) Nanocomposite Materials" Macromolecules, 32, 5765- 71, 1999.

[21] Celzard, A. , J.F. Marêché, J.F., G. Furdin, G. "Modelling of exfoliated graphite" Progress in Materials Science, 50, 93-179, 2005. 
[22] Flandin, L., Bréchet, Y. Canova, G.R. and Cavaillé, J-Y. "AC electrical properties as a senosr of the microstructural evolution in nanocomposite materials: experiment and simulation" Modelling and Simulation in Materials Science and Engineering, 7, 865-874, 1999.

[23] Niklaus, M, and Shea, H.R., "Electrical conductivity and Young's modulus of flexible nanocomposites made by metal-ion implantation of polydimethylsiloxane: The relationship between nanostructure and macroscopic properties" Acta Materialia, 59, 830-840, 2011.

[24] Watson, B.P. and Leath, P.L. "Conductivity in the two-dimensional-site percolation problem" Phys. Rev. B., 9, 4893-4896, 1974.

[25] Straley, J.P., "Critical exponents for the conductance of random resistor lattices" Phys. Rev. B, 15, 5733-5737, 1977.

[26] Yuge, Y. and Onizuka, K., "Monte Carlo experiment for the two-dimensional site percolation network" J. Phys. C: Solid State Phys., 11(18), L763-L765, 1978.

[27] Taya, M. Electronic Composites, Cambridge University Press, Cambridge, UK, 2005.

[28] Blaszkiewicz,M., McLachlan, D.S. and Newnham, R.E. "Study of the volume fraction, temperature and pressure dependence of the resistivity in a ceraminpolymer compoiste using a general effective media theory equation", Journal of Material Science, 26, 5899-5903.

[29] Wu, J., and McLachlan, D.S. "Percolation exponents and thresholds obtained from the nearly ideal continuum percolation system graphite-boron nitride" Phys. Review B, 56(3), 1236-1248, 1997.

[30] Paley, M., and Aboudi, J. "Micromechanical analysis of composites by the generalized cells models" Mech. Mat., 14, 127-139, 1992.

[31] Mori T. and Tanaka K. "Average stress in matrix and average elastic energy of materials with mis-fitting inclusions" Acta Mettallurgica, 2, 571-4, 1973.

[32] Benveniste Y. "A new approach to the application of Mori-Tanaka theory in composite materials" Mechanics of Materials 6, 147-57, 1987.

[33] Aboudi, J. Micromechanics of Composite Materials Studies in Applied Mechanics, Elsevier, NewYork, 1991. 
[34] Snipes, J.S., Robinson, C.T., and Baxter, S.C."Close-Packed and Connected Microstructures: Effects on Mechanical Response of Polymer Nanocomposites" submitted to International Journal of Solids and Structures, 2011.

[35] Pindera, M.-J., and Bednarcyk, B. A. "An efficient implementation of the generalized method of cells for unidirectional multi-phased composites with complex microstructure" Composites Part B (Engineering), 30(1), 87-105, 1999.

[36] Baxter, S.C. and Graham, L.L. "Characterization of Random Composites Using Moving-Window Technique" Journal of Engineering Mechanics 126(4) 389-397, 2000 .

[37] Baxter, S.C. and Graham, L.L. "Simulation of local material propertiesbasedonmoving-windowGMC" Probabilistic Engineering Mechanics, 2001.

[38] Baxter, S.C., Hossein, M.I. and Graham, L.L. "Micromechanics based random material property fields for particulate reinforced composites" International Journal of Solids and Structures, 38, 9209-9220, 2001.

[39] Graham-Brady, L.L, Siragy, E.F. and Baxter, S.C "Analysis of Heterogeneous Composites Based on Moving-Window Techniques" Journal of Engineering Mechanics, 129,9,1054- 1064, 2003.

[40] Blum, L. and Stell, G. "Polydisperse systems. I. Scattering function for polydisperse fluids of hard or permeable spheres" J. Chem. Phys. 71, 42-46, 1979.

[41] DeSimone, T., Stratt, R.M., and Demoulini, S. "Continuum percolation in an interacting system: Exact solution for the Persus-Yevick equation for connectivity in liquids" Phys. rev. Lett. 56, 1140-1143, 1986.

[42] Ostoja-Starzewski M. "Material spatial randomness: From statistical to representative volume element" Probabilistic Engineering Mechanics 2006;21:112-32.

[43] Sau, T. K., Murphy, C. J. "Room Temperature, High-Yield Synthesis of Multiple Shapes of Gold Nanoparticles in Aqueous Solution" J. Am. Chem. Soc., 126, 8648-8649, 2004.

[44] Sisco, P. N., Murphy, C. J. "Surface Coverage Dependence of Surface-Enhanced Raman Scattering from Gold Nanocubes on Self-Assembled Monolayers of Analyte" J. Phys. Chem. A , 113, 3973-3978, 2009. 
[45] Lotters, J.C. Olthuis, W., Veltink, P.H. and Bergveld, P. "The mechanical properties of the rubber elastic polymer polydimethylsiloxane for sensor applications" J. Micromech. Microeng. 7, 145-147, 1997.

[46] Tandon, G.P, and Weng, G. J. "The effect of aspect ratio of inclusions on the elastic properties of unidirectionally aligned composites" Polymer Composites, 5, 327-333, 1984. 


\section{Appendix A}

\section{Matlab Simulation Analysis Code}

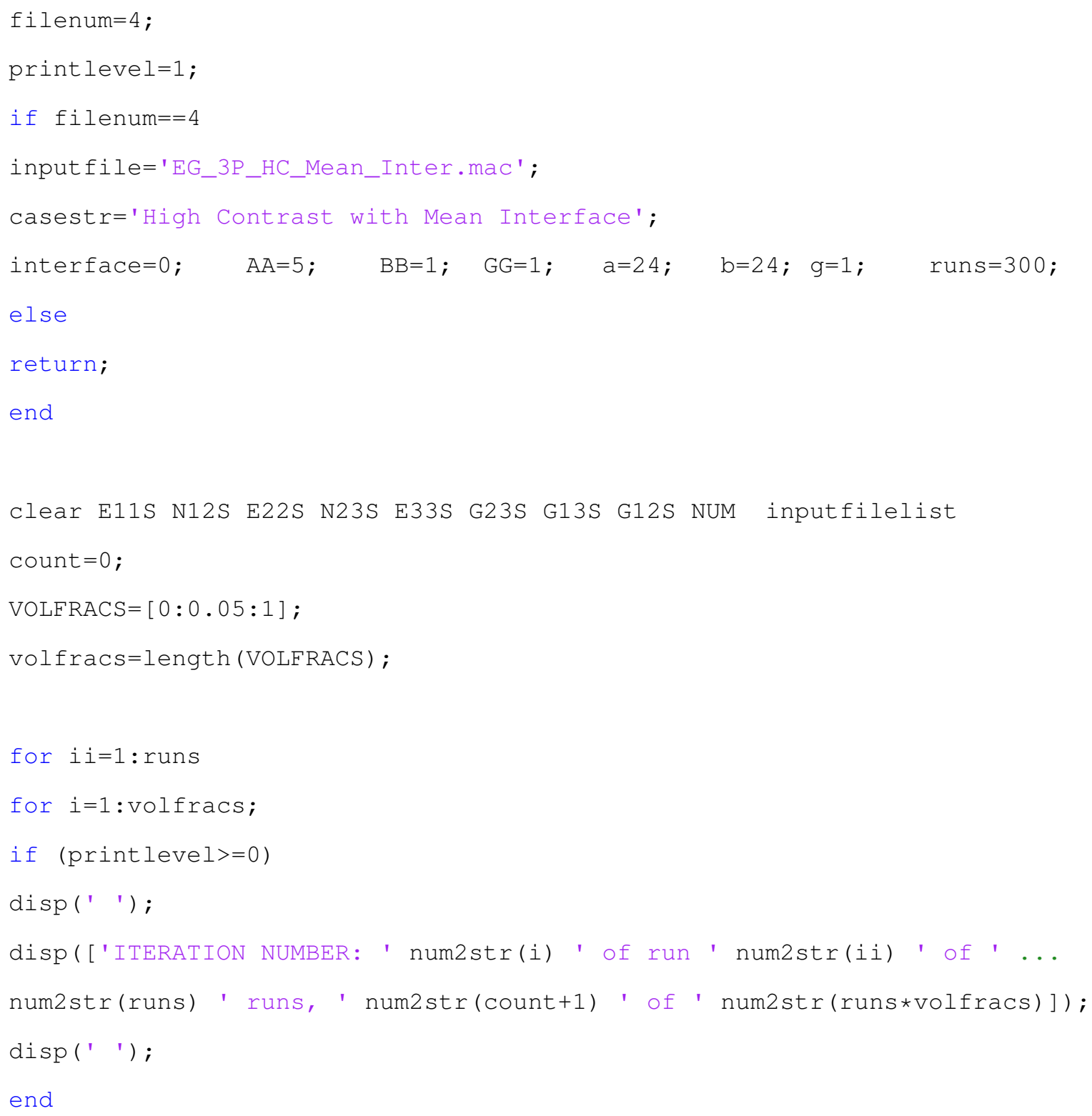




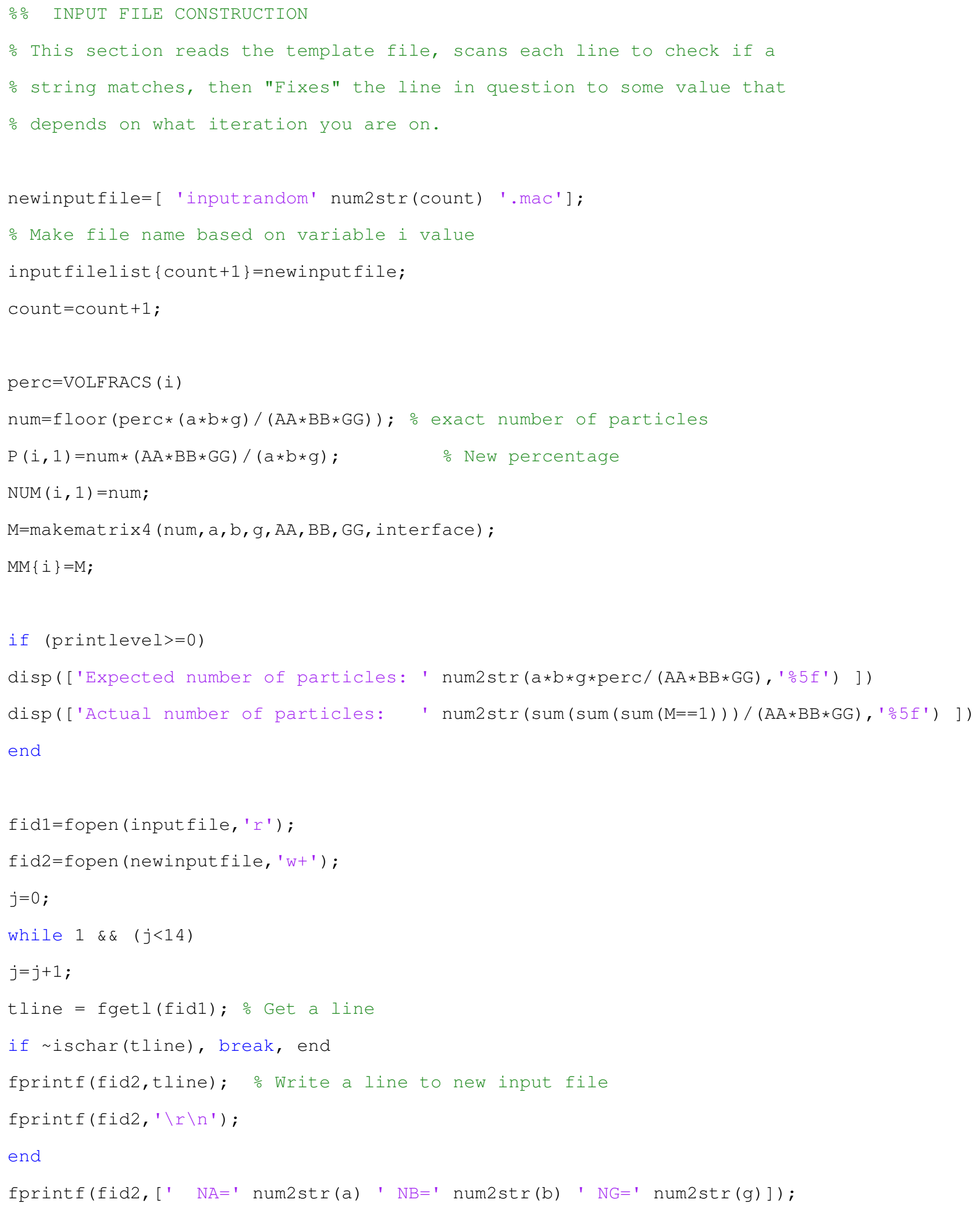




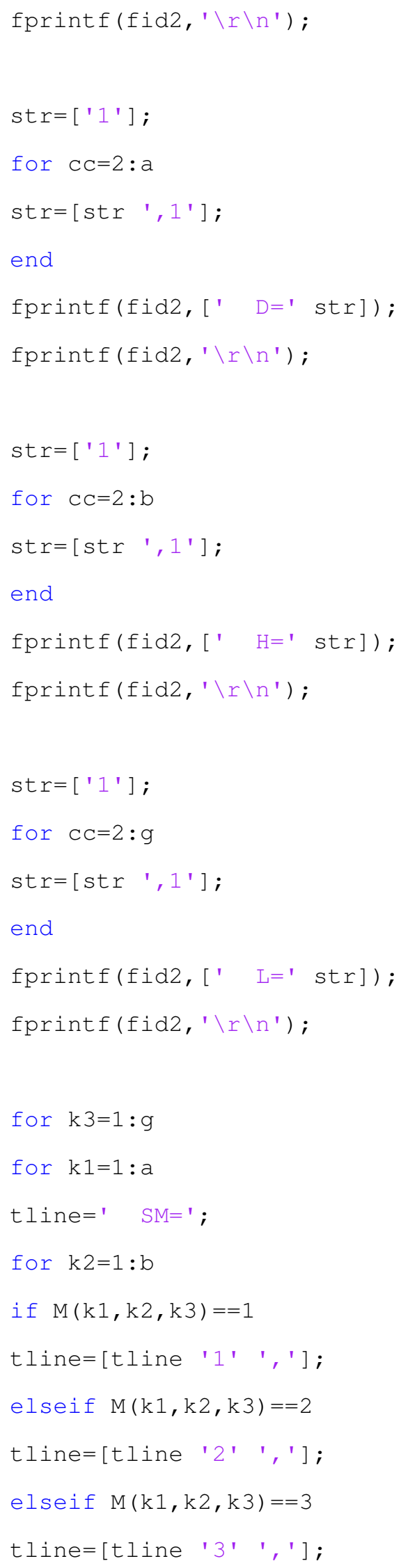




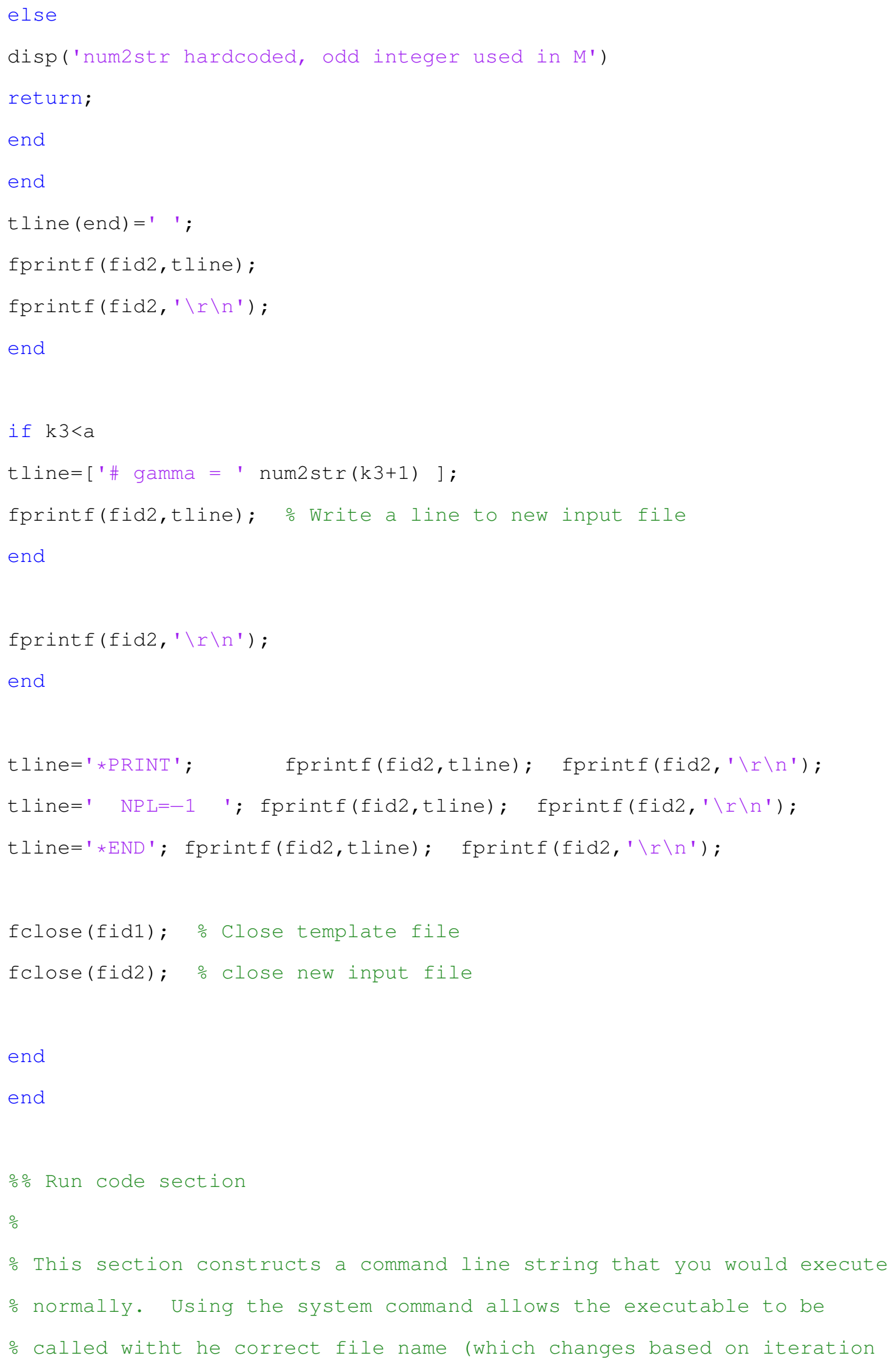


응 ner)

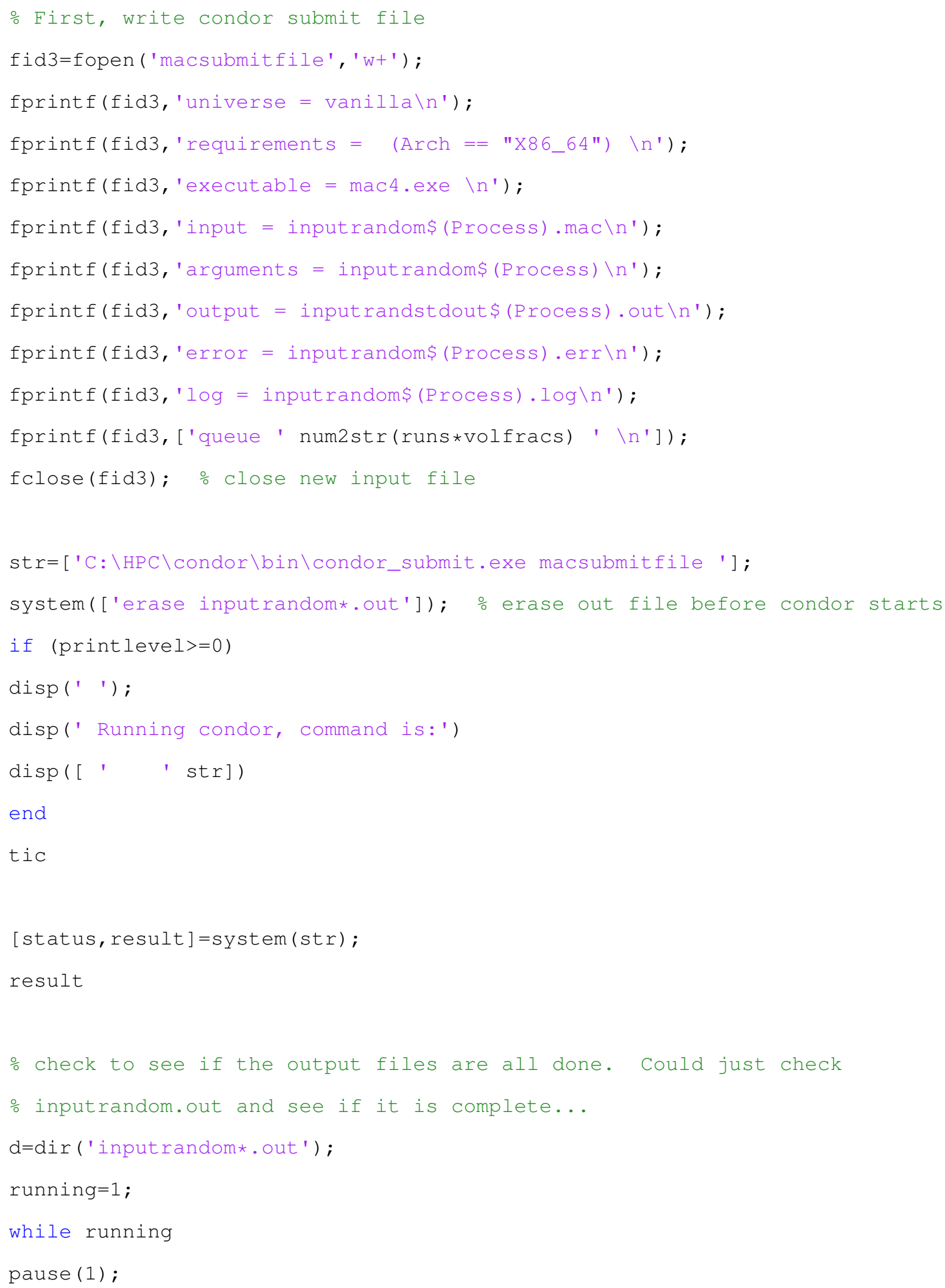




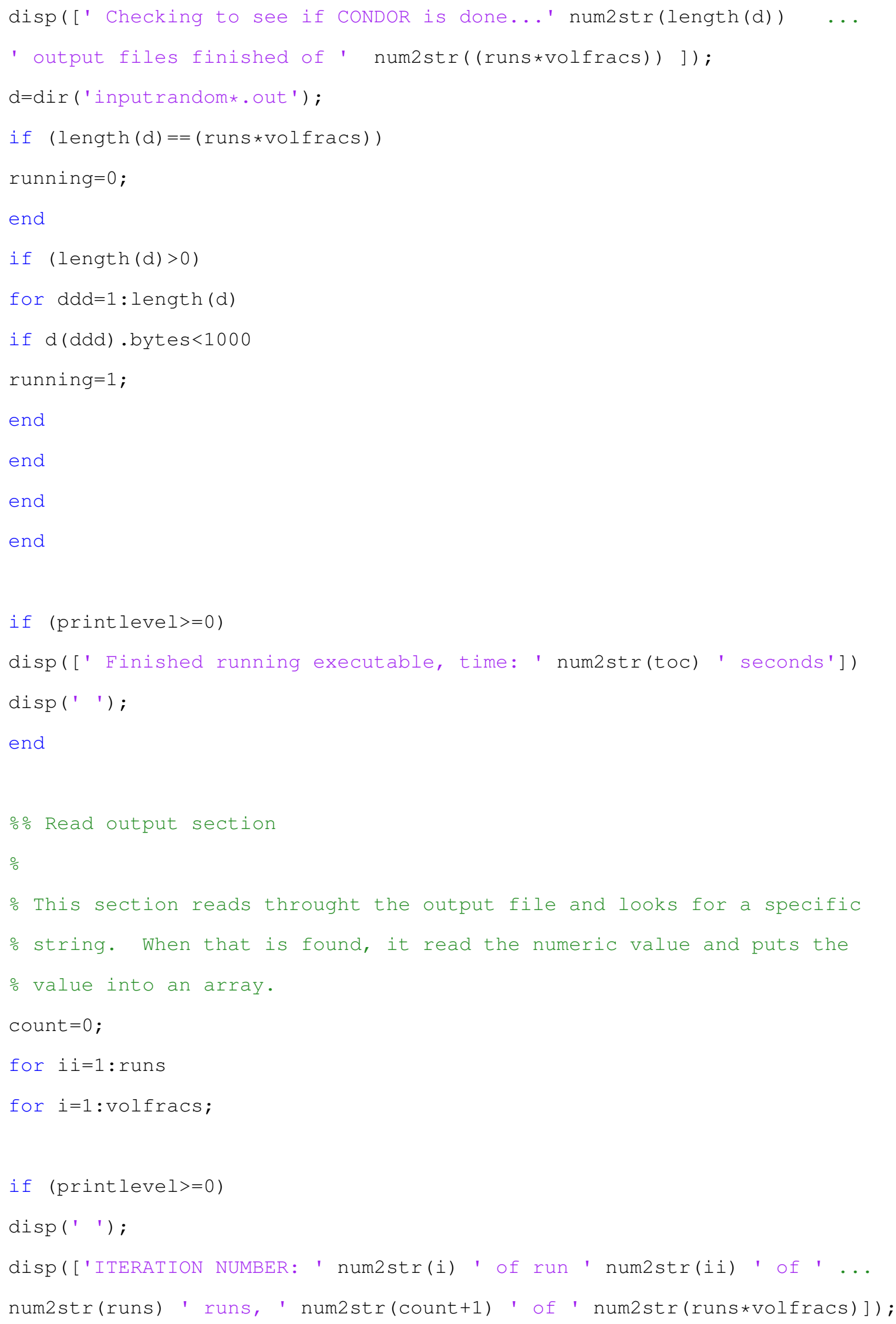




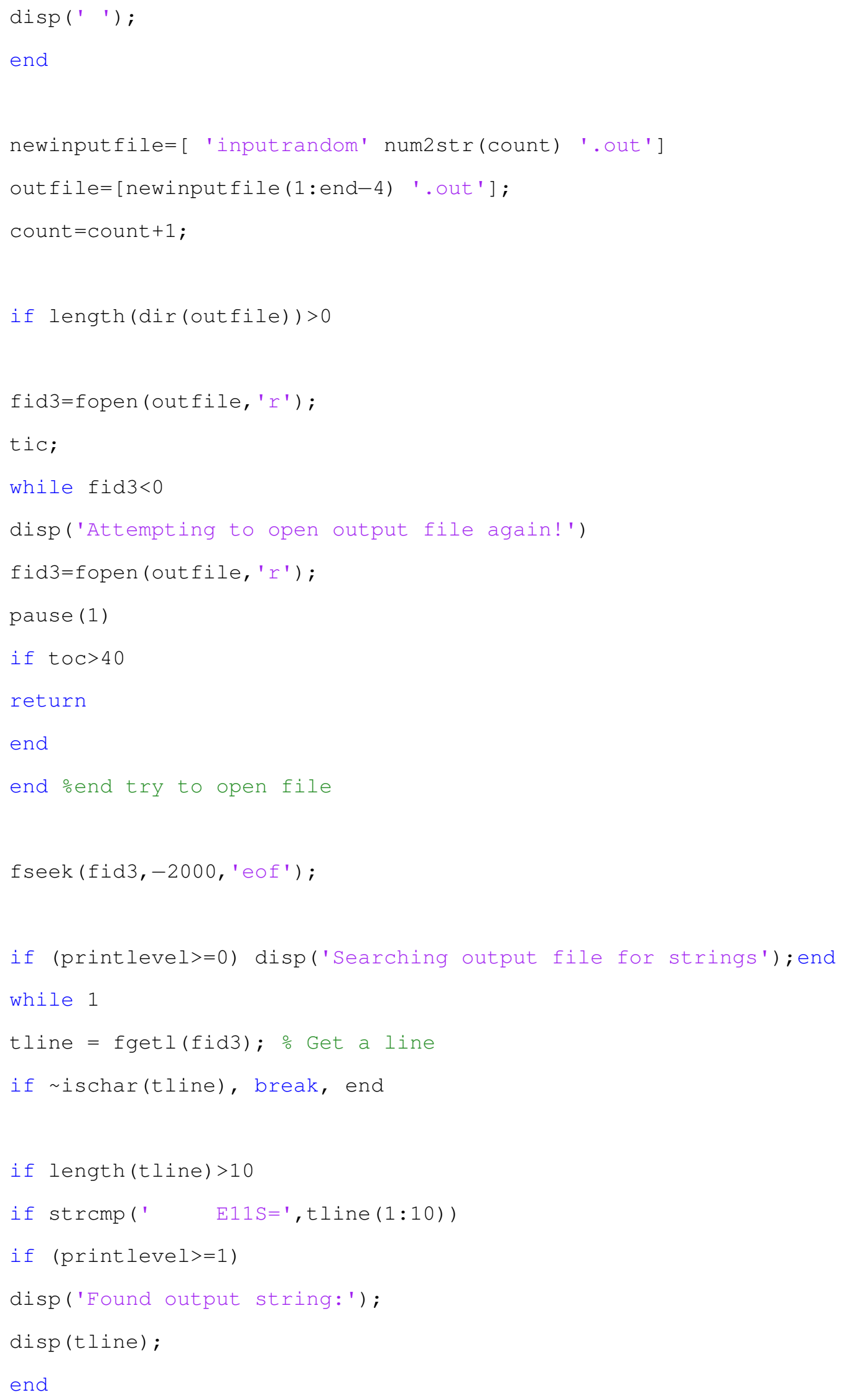




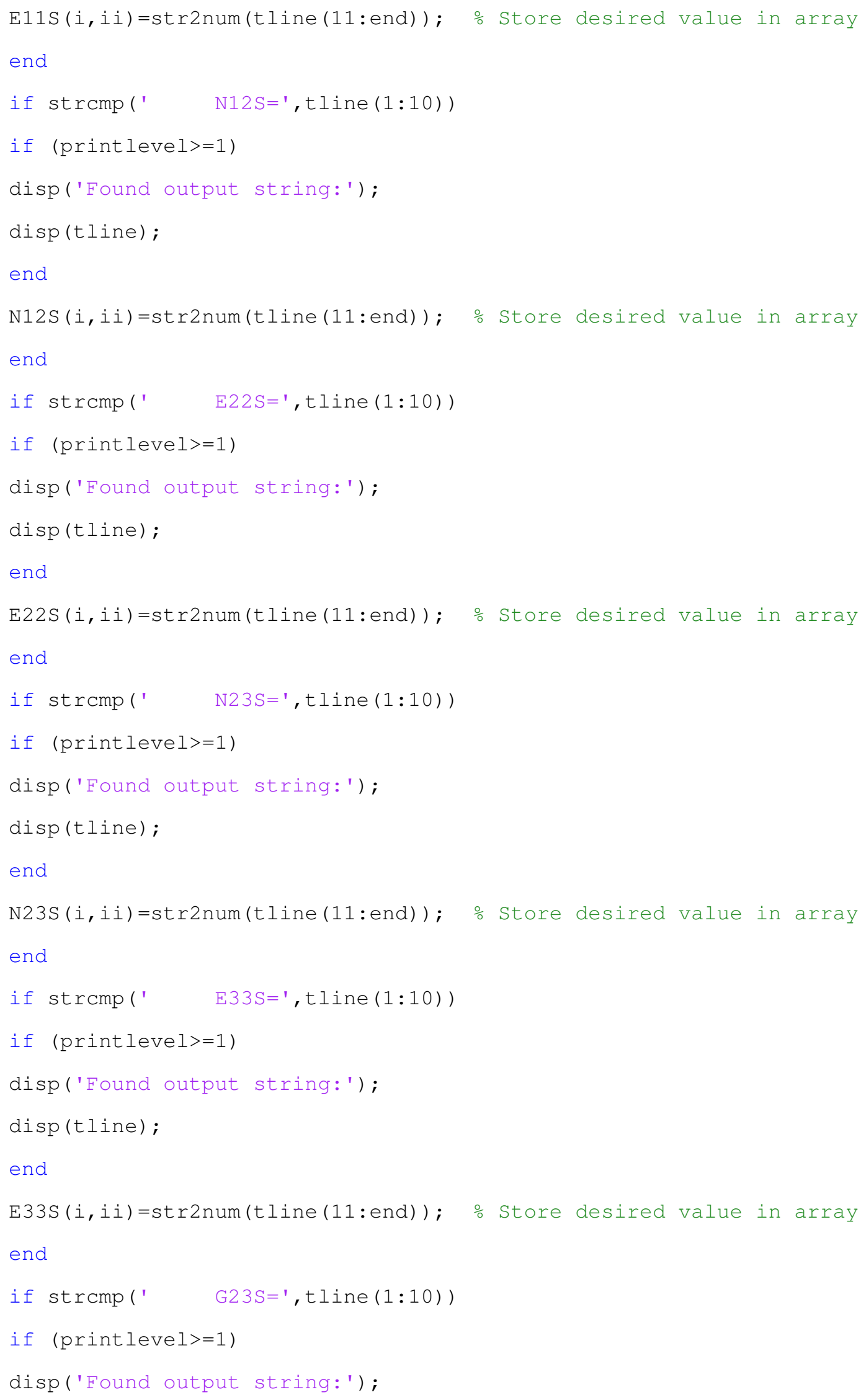




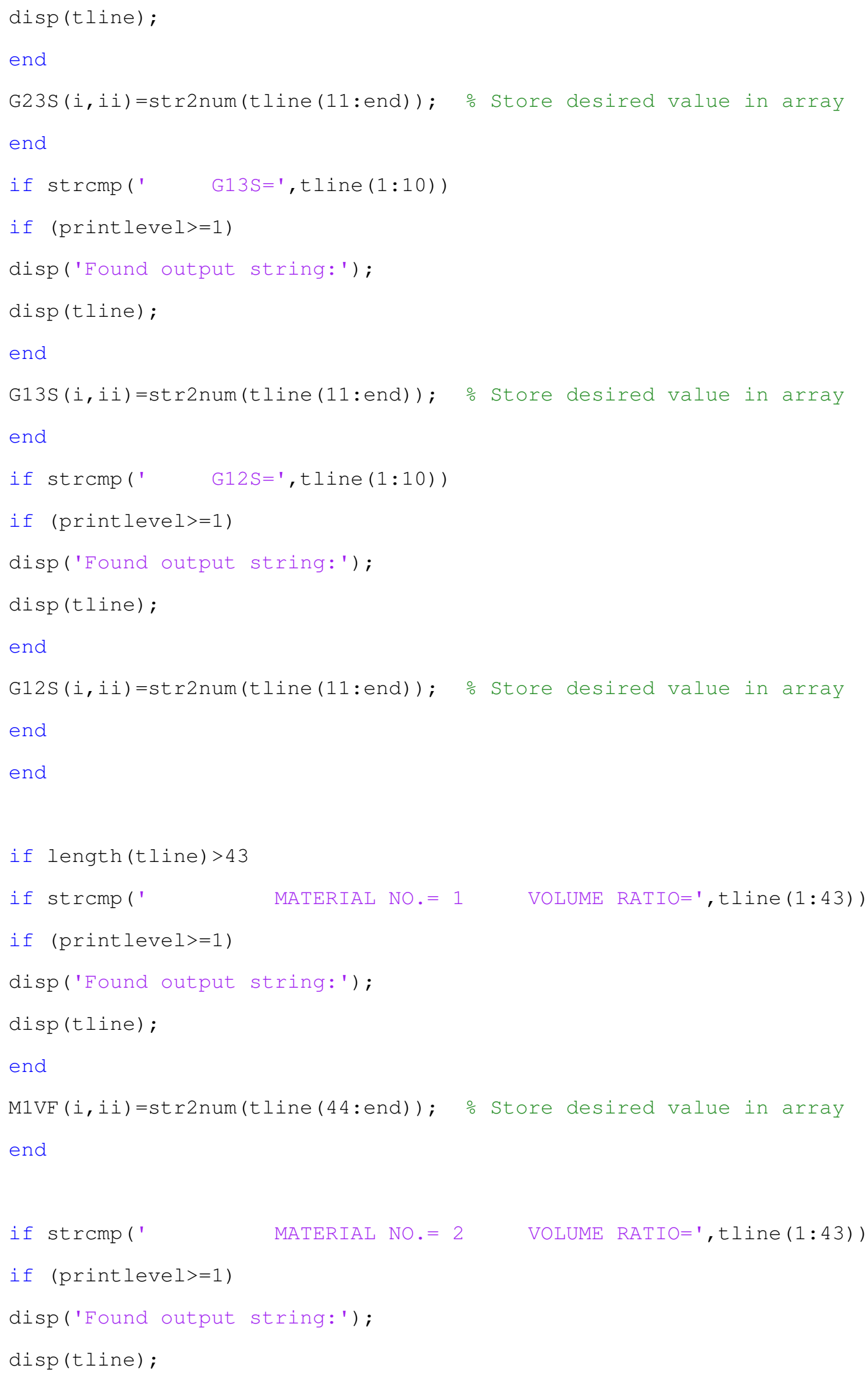




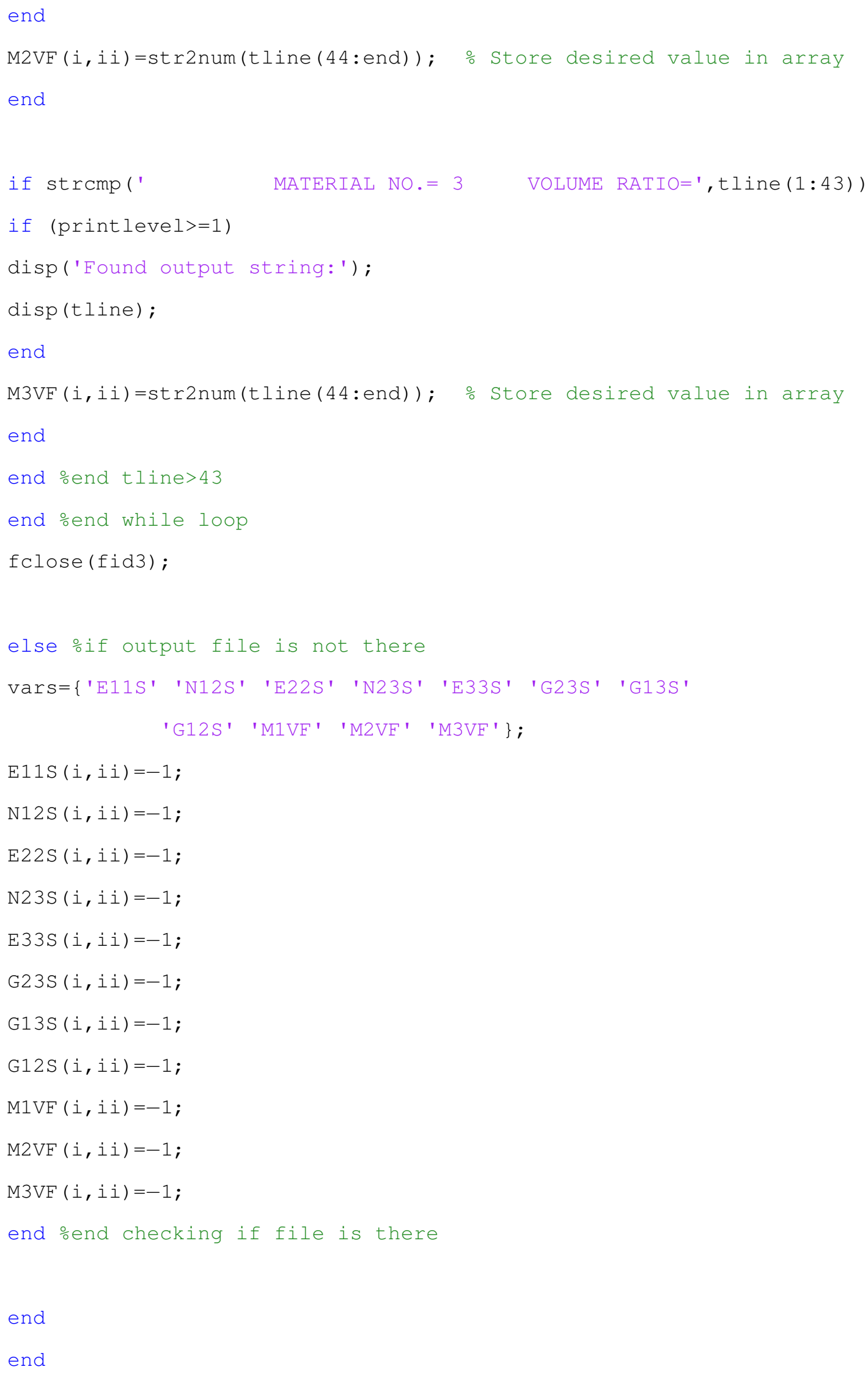




\section{Appendix B}

\section{Matlab RUC Generation Code}

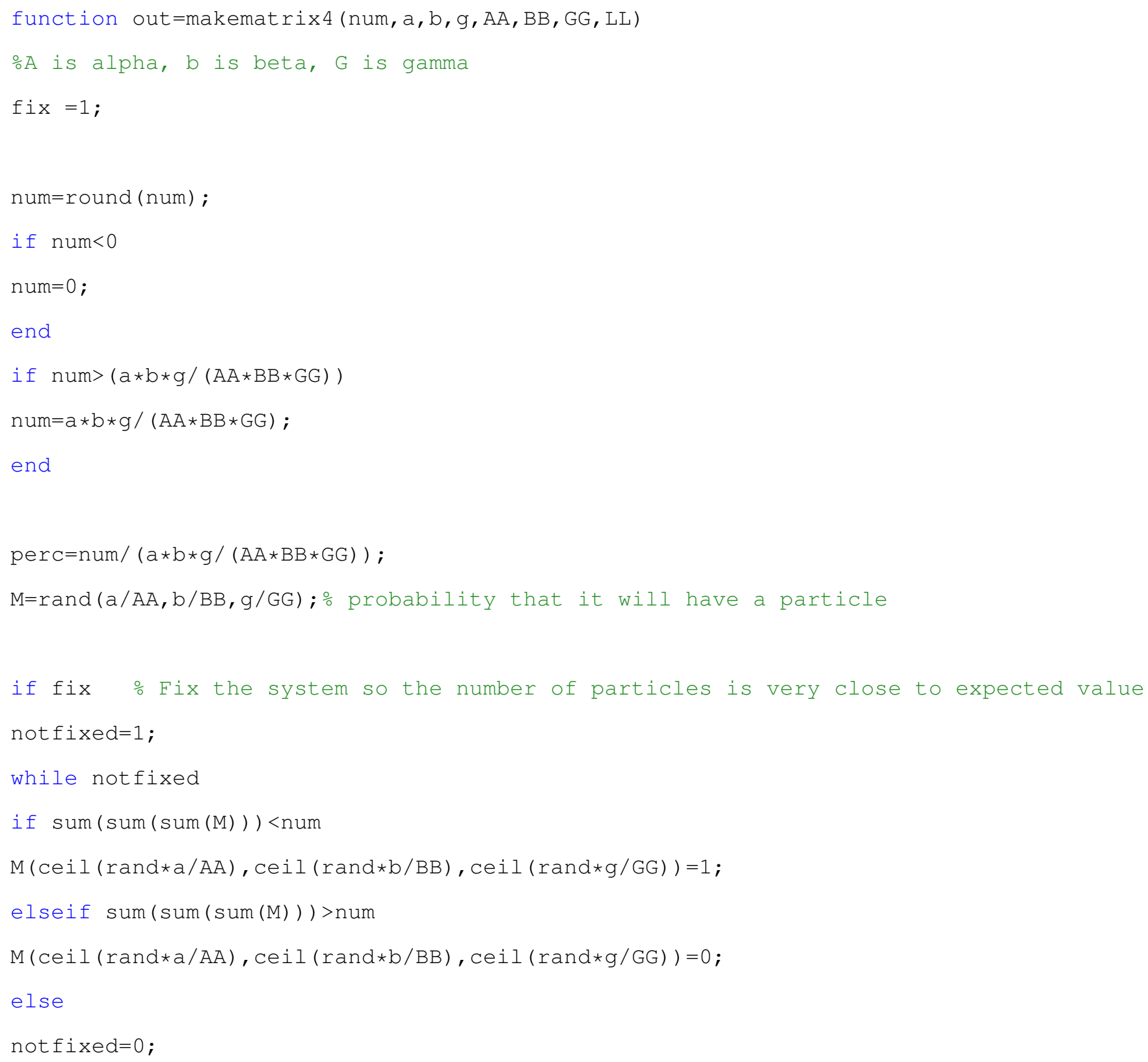




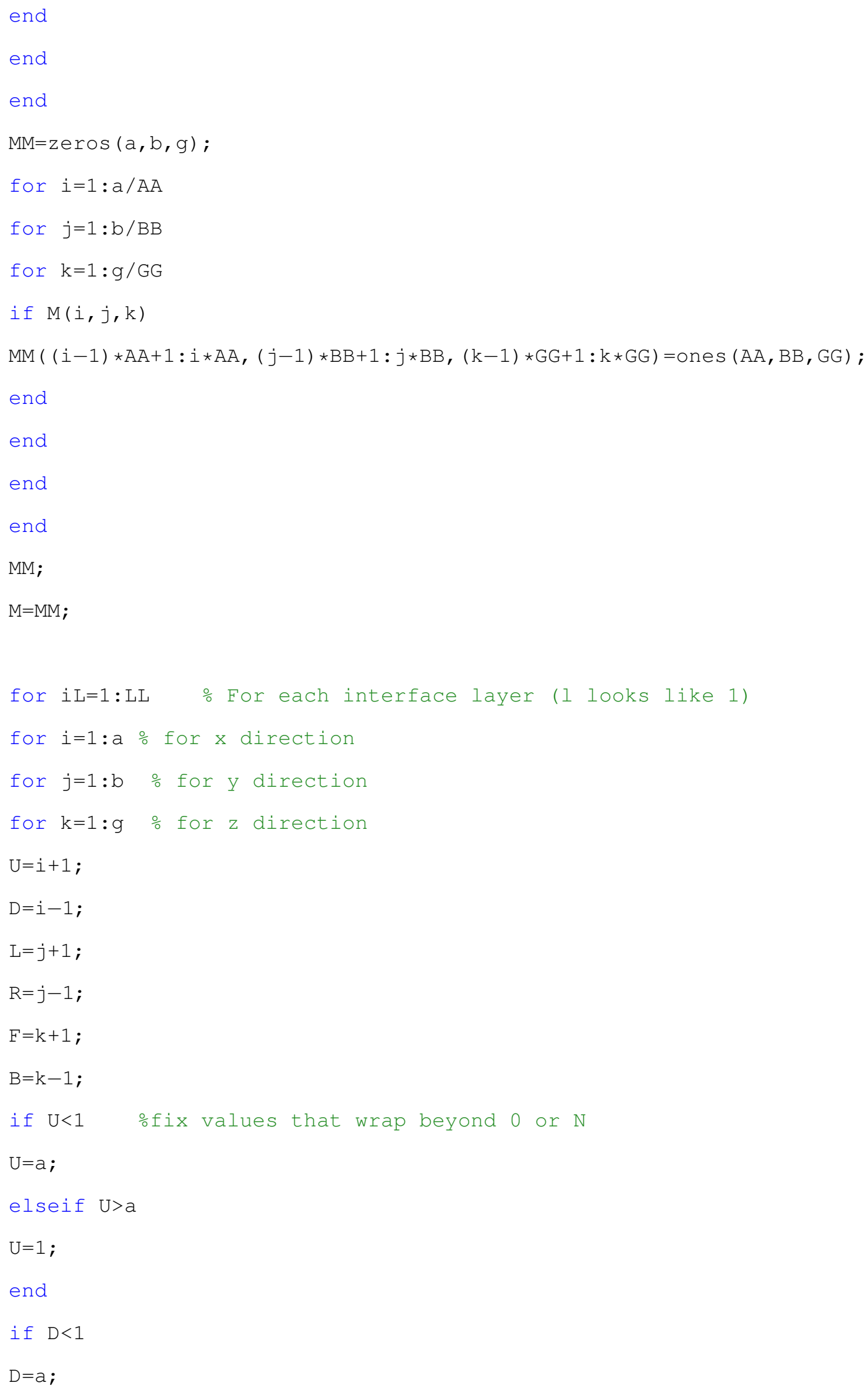




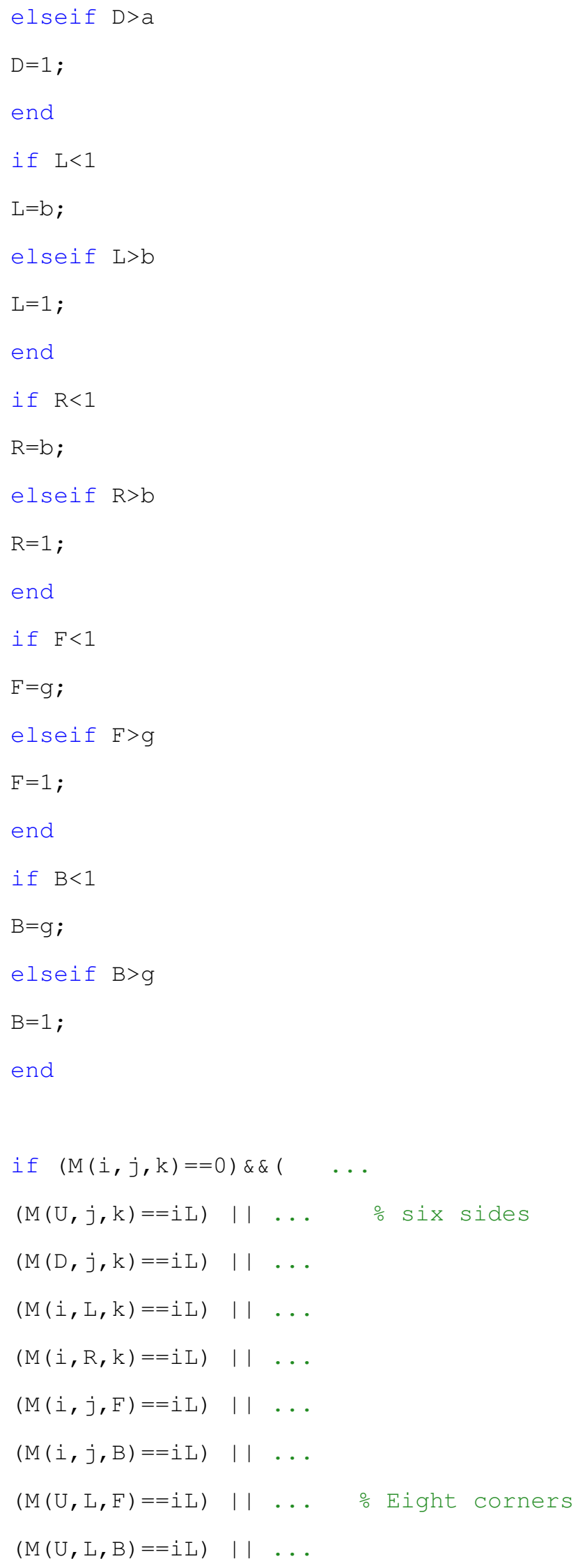




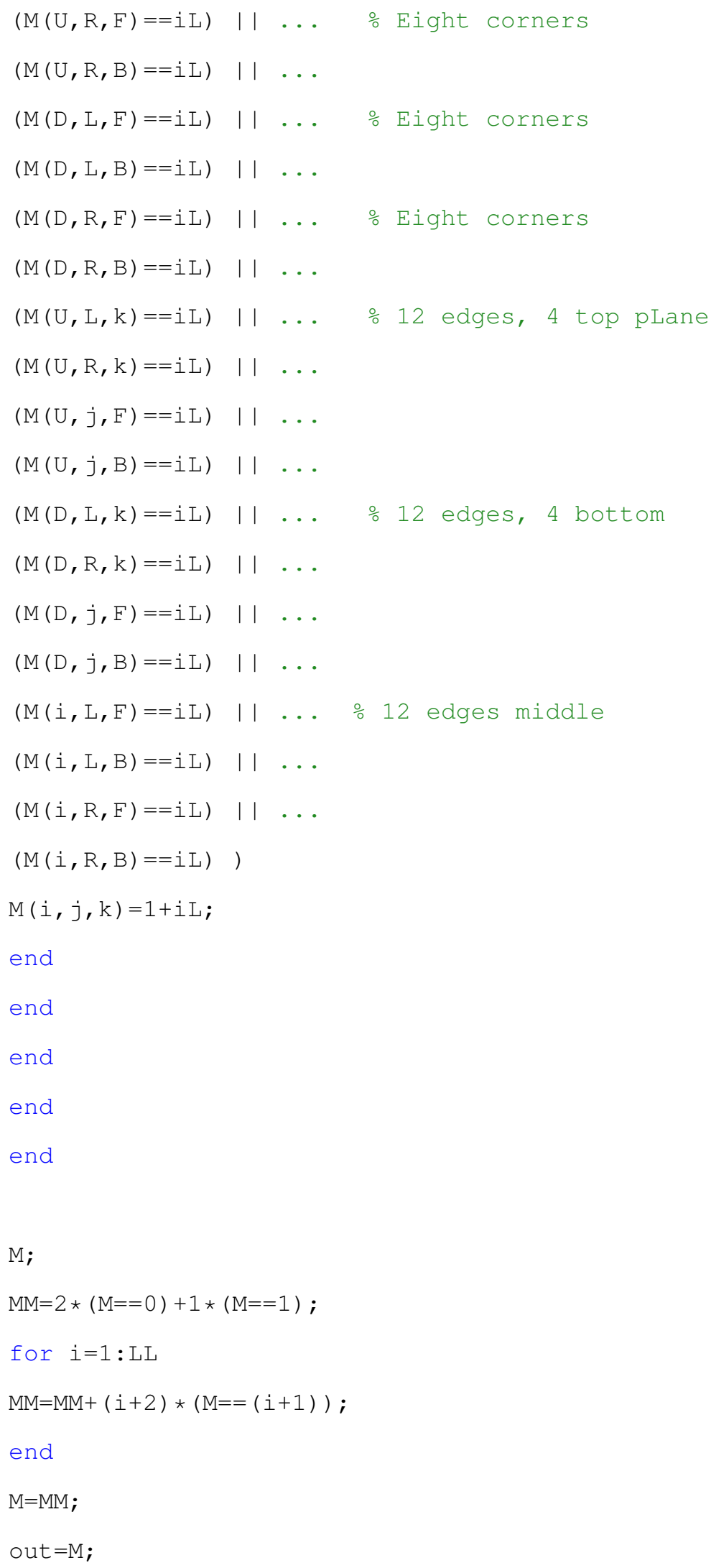




\section{Appendix C}

\section{ExAmple GMC Input Code}

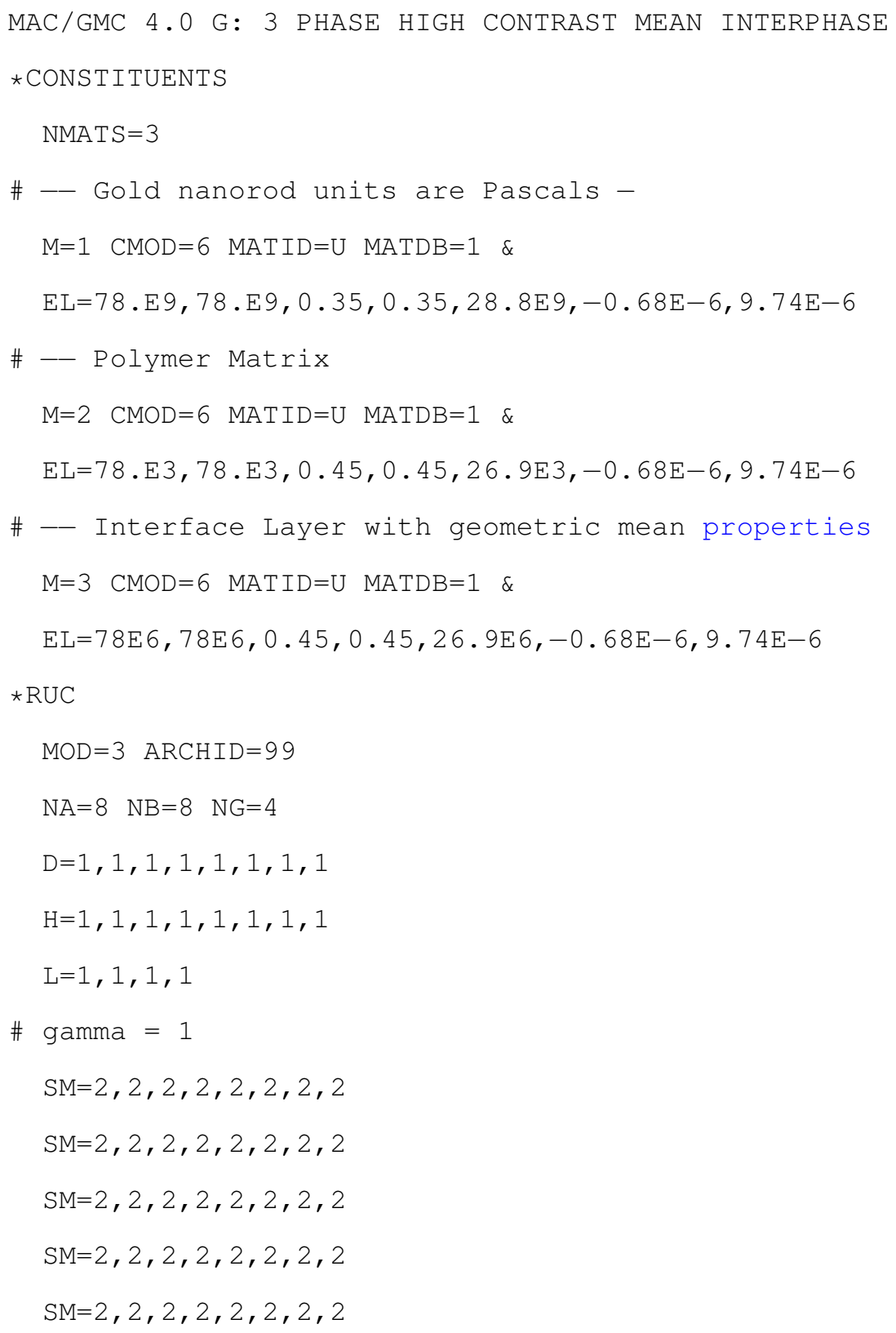




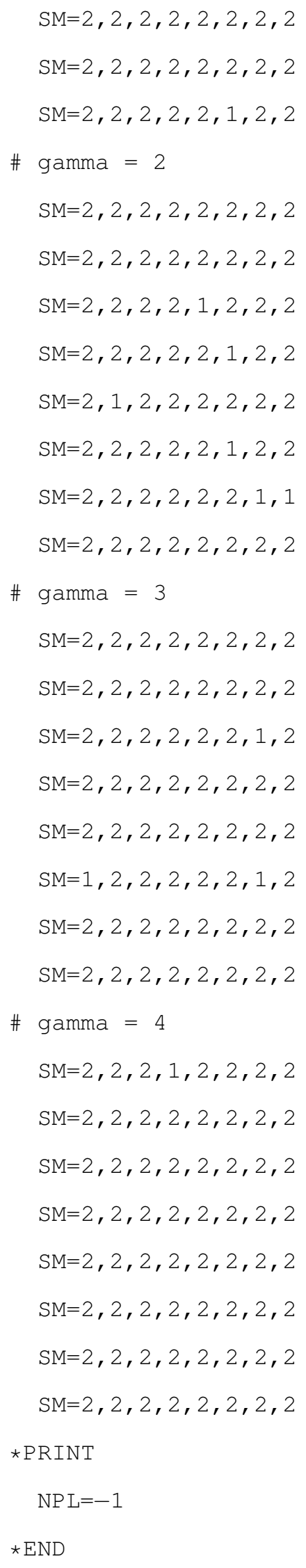

